NBER WORKING PAPER SERIES

\title{
PATIENT VS. PROVIDER INCENTIVES IN LONG TERM CARE
}

\author{
Martin B. Hackmann \\ R. Vincent Pohl \\ Working Paper 25178 \\ http://www.nber.org/papers/w25178
}

\author{
NATIONAL BUREAU OF ECONOMIC RESEARCH \\ 1050 Massachusetts Avenue \\ Cambridge, MA 02138 \\ October 2018
}

We thank our discussants Scott Barkowski, Seth Freedman, Jason Hockenberry, Mark Pauly, Maria Polyakova, and Sally Stearns, as well as John Asker, Moshe Buchinsky, Paul Grieco, Eli Liebman, Adriana Lleras-Muney, Volker Nocke, Edward Norton, Jonathan Skinner, Bob Town, Peter Zweifel, and seminar and conference participants at Aarhus University, Claremont McKenna College, University of Delaware, Duke, University of Georgia, Georgia State, Hamburg Center for Health Economics, Indiana University--Purdue University Indianapolis, LSE, University of Maryland, University of Pennsylvania, USC, Penn State, Queen's University, RAND, RWI, Simon Fraser University, Yale, ASHEcon, EuHEA, iHEA, SHESG, TEAM-Fest, and Whistler for helpful comments. Jean Roth and Mohan Ramanujan provided invaluable help with the data. Funding from the National Institute on Aging grant \#P30 AG012810 is gratefully acknowledged. The views expressed herein are those of the authors and do not necessarily reflect the views of the National Bureau of Economic Research.

NBER working papers are circulated for discussion and comment purposes. They have not been peer-reviewed or been subject to the review by the NBER Board of Directors that accompanies official NBER publications.

(C) 2018 by Martin B. Hackmann and R. Vincent Pohl. All rights reserved. Short sections of text, not to exceed two paragraphs, may be quoted without explicit permission provided that full credit, including $\left({ }^{\circ}\right.$ notice, is given to the source. 
Patient vs. Provider Incentives in Long Term Care

Martin B. Hackmann and R. Vincent Pohl

NBER Working Paper No. 25178

October 2018

JEL No. H51,H75,I11,I13,I18,J14

\begin{abstract}
$\underline{\text { ABSTRACT }}$
How do patient and provider incentives affect mode and cost of long-term care? Our analysis of 1 million nursing home stays yields three main insights. First, Medicaid-covered residents prolong their stays instead of transitioning to community-based care due to limited cost-sharing. Second, nursing homes shorten Medicaid stays when capacity binds to admit more profitable out-ofpocket payers. Third, providers react more elastically to financial incentives than patients, so moving to episode-based provider reimbursement is more effective in shortening Medicaid stays than increasing resident cost-sharing. Moreover, we do not find evidence for health improvements due to longer stays for marginal Medicaid beneficiaries.
\end{abstract}

Martin B. Hackmann

Department of Economics

University of California, Los Angeles

8283 Bunche Hall

Los Angeles, CA 90095

and NBER

mbhackmann@gmail.com

R. Vincent Pohl

Department of Economics

Terry College of Business

University of Georgia

310 Herty Drive

Athens, GA 30602

vincent.pohl@gmail.com 


\section{Introduction}

Long-term care (LTC) expenditures are high and rising. In 2013, U.S. LTC spending accounted for $\$ 310$ billion or $1.8 \%$ of U.S. GDP. Since the share of the population aged 85 and over will more than double by 2050, LTC expenditures may increase twofold (Congressional Budget Office, 2013). In light of this increased demand for LTC services, it is critical that public policies align patient and provider incentives to achieve an efficient utilization of LTC. Since more than $50 \%$ of LTC expenditures are covered by Medicaid, developing and expanding cost-effective home and community alternatives to expensive nursing home care is of high policy priority for many state Medicaid programs (Kaiser Family Foundation, 2015).

In this paper, we study how financial patient and provider incentives affect the mode and cost of LTC as well as their health consequences. This setting is of particular interest for at least two reasons. First, LTC services and, in particular, nursing home care are largely paid for using public funds but provided privately. Separating the role of patient and provider incentives is therefore key for the optimal design of Medicaid policies, which affect utilization of services through cost-sharing and reimbursement regulations. Second, and in addition to the significant spending implications, refining current policies may have important implications for the health of a particularly vulnerable elderly population.

Motivated by the policy context, we focus our analysis on the substitution between nursing home and community based care. ${ }^{1}$ Specifically, we study the timing of nursing home discharges to the community. More than $40 \%$ of nursing home stays end with a discharge to the community, suggesting that community based care is a feasible alternative for a significant fraction of residents. ${ }^{2}$ The precise timing of discharges is largely at the discretion of the nursing home discharge manager and the patient (or her relatives), so it is plausible that economic incentives affect LTC utilization at this margin.

A key advantage of this setting is that we can exploit two sources of plausibly exogenous variation in patient and provider incentives in one context. On the patient side, we exploit variation in out-of-pocket prices among residents who spend down their assets and transition to Medicaid during their nursing home stay. These residents transition from paying the full private rate (set by the nursing home) out-of-pocket to no co-pay under Medicaid coverage. ${ }^{3}$ On the provider side, we exploit variation in the fraction of occupied beds, which affects the nursing home's incentive to discharge Medicaid beneficiaries. At low occupancy rates,

\footnotetext{
${ }^{1}$ LTC covers a variety of services which help to meet the heterogeneous medical and non-medical needs of typically elderly people with varying degrees of physical or cognitive disabilities.

${ }^{2}$ Residents who are discharged to the community have shorter stays on average. Weighted by the length of stay, only about $8 \%$ of seniors are discharged to the community.

${ }^{3}$ In contrast, Medicaid support for community based LTC, which includes services such as home health aides and adult day care, is less generous, particularly so in our sample period from 2000 to 2005.
} 
nursing homes benefit from longer Medicaid stays if the per-diem reimbursement rate, paid by Medicaid, exceeds the marginal cost. At high occupancy rates, when the capacity constraint is binding, nursing homes can benefit from discharging Medicaid beneficiaries in order to admit more profitable new residents who pay the private rate out-of-pocket.

We investigate these effects using micro data from the Long Term Care Minimum Data Set (MDS) combined with Medicaid and Medicare claims data. Our data provide detailed admission, discharge, and health profile information on the universe of nursing home residents in California, New Jersey, Ohio, and Pennsylvania from 2000 to 2005. This allows us to focus our analysis on a relatively homogenous population of residents who pay out-of-pocket at the beginning of their nursing home stay in order to isolate the role of financial incentives on resident discharges. Using the detailed Medicaid claims data, we can identify the exact timing of the transition from private pay to Medicaid coverage and test for a reduction in the weekly discharge rate immediately after the start of Medicaid coverage. We observe such a payer type transition in about $10 \%$ of our sample population of residents. Information on the universe of admission and discharge records allows us to construct and exploit week-to-week variation in the fraction of occupied beds. To isolate the effect of financial incentives, we condition on nursing home-year fixed effects, which absorb confounding variation in staffing and pricing, as well as week-of-stay fixed effects, which flexibly control for duration dependence.

We start with a descriptive analysis on how patient and provider financial incentives impact discharges to the community. At low occupancies, when providers have little incentive to discharge Medicaid or private patients, weekly Medicaid discharge rates are less than half as large as private discharge rates. This suggests that patient financial incentives affect the length of stay. As the occupancy rate increases towards full capacity, we see an economically and statistically significant increase in the discharge probability for Medicaid beneficiaries but no effect for residents who pay out-of-pocket, suggesting that provider incentives also influence the length of stay. We complement the analysis with rich cross-sectional variation in private and Medicaid rates between nursing homes to corroborate these findings. Consistent with incentives tied to patient moral hazard, we find larger differences in discharge rates at low occupancies among nursing homes that charge higher private rates. Turning to the provider incentives, we observe larger increases in Medicaid discharge rates at high occupancies among nursing homes with higher private rate markups over the Medicaid rate. These findings are supported by an extensive list of robustness checks which revisit the findings in a refined sample populations, explore alternative sources of cost-sharing, and allow for forward looking patients, and potential cream-skimming at admission.

The evidence on patient and provider incentives raises the question whether shortening nursing home stays has an adverse effect on patient health. Overall, we find that the residents 
who are at the margin of being discharged to the community are relatively healthy and have low LTC needs. Further, we find no evidence that shorter nursing home stays (on the margin) lead to increases in hospitalization or mortality rates or worsened health status at discharge. These findings suggest that Medicaid beneficiaries are not discharged "too early" at high occupancy rates but point towards substantial overspending. Comparing Medicaid spending on SNF care to the cost of home and community based services (including opportunity costs of informal caregivers), we find that overall LTC spending could be reduced by at least $\$ 1$ billion per year if Medicaid-covered stays were reduced to the average length of stay among residents who pay out-of-pocket, or by about 7.6 weeks on average.

Building on the documented link between financial incentives and discharge profiles, we develop and estimate a dynamic structural model of nursing home discharges. The purpose of the model is to quantify the relative importance of patient and provider incentives in a joint framework. To characterize provider incentives, we require a structural model that allows us to quantify the option value of an empty bed. In addition, we use the structural model to simulate policy counterfactuals that change patient cost-sharing or provider reimbursements. We consider a representative nursing home discharge manager and a patient who is either covered by Medicaid or pays out-of-pocket in the given period (week). ${ }^{4}$ Both sides can exercise costly effort to shorten the length of stay. ${ }^{5}$ Providers optimal effort decision is determined by between the flow payoff of keeping the patient against the option value of admitting a more profitable payer type in the future. Patients trade off utility from nursing home and community based care.

To estimate the structural parameters governing the discharge process, we match the discharge profiles predicted by the model to those derived in the preliminary analysis. Using the estimated model parameters, we simulate the patient and provider elasticity of length of stay with respect to a change in the out-of-pocket price and the Medicaid reimbursement rate, respectively. We obtain a provider elasticity of 1.6 and a patient elasticity of 0.5 , indicating that providers react more elastically to financial incentives than patients.

Building on the estimated model, we revisit the role of patient and provider incentives in several policy counterfactuals. Motivated by the German long term care reform from 1995, we first simulate the effects of increases in patient cost-sharing on the length of nursing home stays. ${ }^{6}$ Our simulation results show that a LTC voucher system, under which Medicaid

\footnotetext{
${ }^{4}$ We adopt two strategies to address patient and provider heterogeneity. In our baseline analysis, we purge the data off from patient and provider heterogeneity and estimate the model to the residualized data. In robustness exercises, we split the sample into different nursing home populations and compare the estimates between the samples.

${ }^{5}$ This includes resources spent on finding alternative care arrangements as well as preparing the resident for a more independent living arrangement.

${ }^{6}$ The reform introduced lump-sum allowance payments to seniors, which varies in their long term care
} 
beneficiaries pay the full private SNF rate and providers receive the same fee for all payer types, reduces the length of stay by about $24 \%$. However, the policy marginally raises public LTC expenditures due to the lump-sum (voucher) transfers that compensate seniors for their outlays.

In contrast, we find that changing the timing of reimbursements is very effective is shortening Medicaid stays and lowering public spending. Our results indicate that transitioning $10.5 \%$ of current Medicaid per-diem reimbursements to an episode-based (up-front) reimbursement is as effective as the voucher program in shortening the length of Medicaid stays but yields substantially larger annual total cost savings of about $\$ 0.18$ billion. This reimbursement counterfactual is motivated by ongoing experimentation over episode-based Medicare reimbursement models for post-acute nursing home care. ${ }^{7}$

Overall, we find that providers respond more elastically to financial incentives than patients despite the fact that our demand elasticities exceed estimates from the literature, see Manning et al. (1987), Finkelstein et al. (2012), and Shigeoka (2014) for elderly people, which center around 0.2. In fact, most of our robustness exercises indicate that our baseline estimates, if anything, overstate the role of patient incentives, corroborating this qualitative comparison. Changing provider incentives may also be the preferred option to shorten nursing home stays among marginal Medicaid beneficiaries because increasing patient cost sharing reduces the value of risk protection provided by Medicaid insurance, a benefit we do not explicitly incorporate into our model.

Our findings contribute to several literatures. First, we contribute to large literature examining the link between financial incentives and health care utilization in other sectors. While the vast amount of existing work has focused on patient incentives, see Aron-Dine, Einav, and Finkelstein (2013) for an overview, less is known on the role of provider incentives. Previous work has tested whether providers do respond to financial incentives in the context of introduction of Inpatient Prospective Payment System in 1983 (Cutler, 1995; Cutler and Zeckhauser, 2000). More recent studies have investigated behavioral responses of physicians to financial incentives (Clemens and Gottlieb, 2014; Ho and Pakes, 2014). Our supply side analysis provides novel evidence on the link between health care utilization and binding capacity constraints, which is commonly ignored in the existing literature. Perhaps most importantly, we are able to distinguish between patient and provider incentives in our context. This is of particular importance for the design of public insurance programs, which

needs, but required that seniors pay a significant portion of the nursing home price out-of-pocket.

${ }^{7}$ See e.g., the Bundled Payments for Care Improvement (BPCI) initiative under the authorization of the Centers for Medicare and Medicaid services. Findings from pilot studies suggest that moving to an episode-based Medicare reimbursement model for nursing homes can lower Medicare nursing home payments (and overall Medicare spending) without significant detrimental effects on patient health, see Dummit et al. (2018). 
affect the incentives for patients and providers, sometimes in opposing directions. While the significant implications for both market sides has been noted for a long time (see, e.g., Ellis and McGuire, 1993)), the existing empirical literature has largely studied the role of demand and supply-side cost sharing in isolation, see McGuire (2011) for an overview. ${ }^{8}$

Our structural analysis is closely related to the recent supply side analysis in Eliason et al. (forthcoming) and Einav, Finkelstein, and Mahoney (forthcoming). These latter studies find that a discrete jump in Medicare reimbursement changes provider incentives to discharge post-acute care patients from LTC hospitals. Our context deviates from their's in several important ways. First, we focus on different context of enormous spending and policy relevance: the design of Medicaid insurance in long term care. Second, we can leverage detailed health records in the MDS, which allows us to investigate the health effects of shorter SNF stays. Third, we exploit variation in occupancy instead of reimbursement rules as a source of financial provider incentives. The incentives originating from binding capacity constraints apply to other health care industries as well and have implications for the effectiveness of Certificate of Need (CON) laws, which restrict nursing home entry and capacity investment decisions. Finally, our framework incorporates both provider and patient incentives. This allows us to investigate how demand and supply-side cost sharing incentives jointly determine health care utilization.

Second, we contribute to the literature on financial patient and provider incentives in LTC. To the best of our knowledge, we provide the first evidence on the causal effect of provider profit incentives on the length of nursing home stays. ${ }^{9}$ In two descriptive studies, Arling et al. (2011) and Holup et al. (2016) find that facilities with higher average occupancy rates are more likely to discharge residents to the community, but do not differentiate by payer type. Our findings are consistent with their observations and can also reconcile the negative cross-sectional relationship between nursing home occupancy and access to care for Medicaid beneficiaries, see Ching, Hayashi, and Wang (2015). The authors argue that capacity constrained nursing homes cream-skim against Medicaid beneficiaries at admission. In contrast, we document that nursing homes can manage their payer mix through endogenous discharges. While we cannot completely rule out potential cream-skimming at admission, we are less concerned about this channel in our context as we study seniors who pay initially

\footnotetext{
${ }^{8} \mathrm{~A}$ notable exception is Trottmann, Zweifel, and Beck (2012), who study the impact of demand and supply-side cost sharing incentives in Swiss health insurance plans on health care utilization. Also, Dickstein (2015) studies patient and physician incentives in the market for antidepressants. In contrast to our results, Dickstein (2015) finds that more utilization leads to better health outcomes, so the normative implications are not as clearcut as in the case of SNF utilization that we study.

${ }^{9}$ Previous studies have investigated the effect of Medicaid bed-hold policies on the hospital discharges, see (Intrator et al., 2007). In contrast to the community discharges in our study, most residents return from the hospital to the SNF, so the implications for the length of nursing home stays are unclear in these studies.
} 
out-of-pocket and are therefore relatively homogenous at admission.

Finally, our analysis of patient incentives in LTC complements existing evidence which remains mixed and incomplete. An earlier series of studies, known as the Channeling demonstration, found that the community care interventions had relatively small effects on nursing home utilization suggesting very little substitutability among community and nursing home care, see Rabiner, Stearns, and Mutran (1994). Consistent with these results, McKnight (2006) and Grabowski and Gruber (2007) find that the decision to enter a nursing home is relatively inelastic with respect to Medicaid cost-sharing incentives. On the other hand, Konetzka et al. (2014) find that private LTC insurance increases the propensity of nursing home stays and Mommaerts (2017) documents heterogeneous effects of spend-down requirements on SNF entry. Importantly, very little is known about how patient incentives affect the length of nursing home stays. Our findings provide new evidence on this politically and economically important margin. ${ }^{10}$

The remainder of this paper is organized as follows. We describe institutional details on Medicaid and SNFs discharges in the next section. We then develop a theoretical model of provider and patient incentives and nursing home discharges in Section 3. We test the model predictions using the data described in Section 4 and the empirical strategy developed in Section 5. Section 6 presents first direct evidence on the role of patient and provider incentives. Motivated by this evidence, we then develop the structural model of resident discharges and discuss its identification and estimation in Section 7. Section 8 provides the structural estimation results and contains our counterfactual policy simulations. We conclude in Section 9.

\section{Institutional Details}

In this section, we describe select institutional features of the U.S. nursing home industry. We focus on Medicaid eligibility and provider reimbursement, and the discharge management of residents, which are most important for the subsequent analysis.

\subsection{Medicaid Eligibility, Reimbursement, and Cost-Sharing}

Nursing home care is largely financed by Medicaid, covering about $65 \%$ of all nursing home days. $25 \%$ of all days are funded privately with the majority being paid out-of-pocket. Only $16 \%$ of privately funded nursing home days (4\% of all days) are paid for by private LTC insurance (Hackmann, 2017). The remaining 10\% are covered by Medicare which only covers

\footnotetext{
${ }^{10}$ Our findings are supported by previous descriptive studies, which document that Medicaid beneficiaries are on average less likely to be discharge to the community, see Weissert and Scanlon (1985), Chapin et al. (1998), and Gassoumis et al. (2013). These studies lack a clean source of identifying variation and cannot explore the mechanisms reconciling this observation.
} 
post-acute care of up to 100 days. Since the health profiles of residents whose stay is covered by Medicare are very different from Medicaid beneficiaries and private payers, we do not include them in the analysis.

In order to qualify for Medicaid, seniors cannot have assets above a state-specific resource limit of $\$ 2,000$ to $\$ 4,000$ and have to demonstrate LTC need due to medical reasons or functional limitations. States have different level-of-care criteria to determine Medicaid eligibility on medical grounds, but usually a state agency assesses potential beneficiaries for medical conditions that require skilled nursing care and limitations in activities of daily living (ADL). ${ }^{11}$

In addition, Medicaid eligibility is subject to an income test, and beneficiaries with income below the eligibility threshold do not face copayments for SNF care. ${ }^{12}$ It is also possible for seniors above the income limit to qualify for Medicaid under so-called medically needy programs. Under these programs, nursing home residents who pass the asset test can deduct medical expenses, including SNF fees, from their income and qualify for Medicaid if their adjusted monthly income falls below a state-specific limit of about $\$ 400$ to $\$ 600$. In that case, the resident's entire income except for a personal needs allowance of about $\$ 50$ is applied to the cost of nursing home care. ${ }^{13}$ We abstract away from these co-payments in our baseline analysis which can account for at most $9 \%$ of the full nursing home bill. ${ }^{14}$ We revisit the significance of co-payments for our main findings in the robustness check analysis in Section 6.2.

In practice, the asset test is the binding constraint for Medicaid eligibility. Using data from the Health and Retirement Study (HRS), we find that among seniors whose assets are below $\$ 2,000$ and $\$ 4,000$, respectively, only $1 \%$ have income levels that would make them ineligible for Medicaid under a medically needy program. Moreover, Borella, De Nardi, and French (2017) find that among seniors in their 90s, up to $60 \%$ in the bottom permanent income tercile receive Medicaid benefits. In the middle permanent income tercile, over $20 \%$ become eligible for Medicaid as they age. This indicates that Medicaid coverage becomes widespread as seniors age and spend down their assets.

While some nursing home residents qualify for Medicaid at the beginning of their stay, our analysis focusses on residents who are initially ineligible for Medicaid because their assets

\footnotetext{
${ }^{11}$ Of the states in our sample, California requires both ADL limitations and a medical condition that necessitates 24-hour supervision while one of the two requirements is sufficient in New Jersey and Ohio, and in Pennsylvania nursing needs are a necessary condition for Medicaid eligibility (see O'Keeffe, 1999).

${ }^{12}$ See, e.g., https://www.medicaid.gov/medicaid/ltss/institutional/nursing/index.html.

${ }^{13}$ See https://kaiserfamilyfoundation.files.wordpress.com/2014/07/medicallyneedy2003.pdf.

${ }^{14}$ We find an average monthly income of $\$ 625$ among Medicaid beneficiaries using data from the National Long Term Care Survey. Considering a net allowance of $\$ 50$ per month and an average private rate of $\$ 218$ per day, this suggests that Medicaid beneficiaries pay at most $\frac{\$ 625-\$ 50}{\$ 218 \times 30 \text { days }}=9 \%$ of what private payers pay.
} 
exceed the eligibility threshold. About $10 \%$ of these residents spend down their assets during their stay and thereby switch from out-of-pocket pay to Medicaid coverage. Once residents have depleted their assets and become eligible for Medicaid, their price of nursing home care drops sharply. In Pennsylvania, for example, average private rates were $\$ 218$ per day. At the same time, Medicaid beneficiaries who were discharged from a nursing home during our sample period (2000 to 2005) had to pay for home health care mostly out of pocket. ${ }^{15}$ Taken together, these incentives favor prolonged nursing home stays among Medicaid beneficiaries.

Medicaid pays nursing homes a regulated, risk-adjusted, daily reimbursement rate that is usually lower than the out-of-pocket (private) rate. In Pennsylvania, for example, Medicaid reimbursement rates averaged $\$ 188$ in our sample period. At the same time, federal and state legislation, such as the Omnibus Budget Reconciliation Act of 1987, prohibits nursing homes from offering different quality of care levels by payer source. Grabowski, Gruber, and Angelelli (2008) find that SNFs comply with this regulation, so nursing homes generate lower profits per Medicaid resident than per private payer conditional on LTC needs. Despite lower fees, Medicaid beneficiaries are generally profitable for nursing homes because reimbursement rates exceed the marginal cost of care (Hackmann, 2017).

Finally, several nursing homes cannot change their bed capacity at least in the short run because of fixed costs of investments but also because many states require nursing homes to obtain a CON in order to increase the number of beds. ${ }^{16}$

\subsection{Nursing Home Discharges}

Nursing home discharges occur in one of the following ways. If residents' health improves and their LTC needs decline, nursing homes may discharge them to the community (e.g to a private or retirement home). This is the most common discharge reason for nursing home stays in our sample population. At home these seniors may then seek help from home health agencies or informal caregivers for their activities of daily living. If their LTC needs do not decline, the nursing home stay can end because the resident was discharged to a hospital, a different nursing home, or an assisted living facility, or because the resident passed away. ${ }^{17}$ In our empirical analysis, we view the latter types of "non-community" discharges as

\footnotetext{
${ }^{15}$ For an overview of Medicaid home and community based services (HCBS), see Appendix Section A.

${ }^{16}$ Of the states in our sample, New Jersey, Ohio, and Pennsylvania had CON laws between 2000 and 2005 while California did not. Some states, including Pennsylvania, only limit the number of SNF beds that can be occupied by Medicaid beneficiaries.

${ }^{17}$ Discharges due to hospitalizations are also affected by bed-hold policies. Since Medicaid reimburses nursing homes for keeping a bed vacant while a resident is hospitalized, SNFs may have a financial incentive to temporarily discharge Medicaid residents to a hospital (Intrator et al., 2007). Our analysis focuses on permanent discharges when a return to the nursing homes is not expected. In other words, potential temporary discharges to a hospital will not interrupt our nursing home stay measure and will neither affect our measured occupancy rate nor the actual rate as nursing homes must keep the bed vacant. We also provide
} 
"exogenous" in the sense that they are due to medical reasons, whereas community discharges may be affected by the SNFs and residents' financial incentives.

For seniors who have spent some time in a nursing home where they rely on around-theclock care, transitioning into the community poses substantial challenges. Specifically, the management of complex medical conditions, support from family members or other informal caregivers, and housing that is adapted to the senior's medical conditions need to be arranged (see, e.g., Meador et al., 2011). Nursing home residents who prefer returning home and their relatives therefore have to exert a considerable effort before a discharge is possible.

From the nursing home's perspective, discharge decisions are made jointly with the resident and her family, and SNFs regularly evaluate their residents' health status to determine if they should remain in the facility or be discharged to the community. Nursing homes also assist residents who express a wish to return home with the above-mentioned tasks. According to discharge managers whom we interviewed, nursing homes have no systematic rules for when to discharge a resident, however. For example, discharge decisions are not tied to a certain case mix index (CMI) value or other objective health outcomes (see Appendix Section $\mathrm{C}$ for details on the CMI). While discharge managers asserted that current occupancy rates or waiting lists do not play a role in deciding a resident's discharge, no formal mechanism prevents such behavior.

Although federal regulations such as the Nursing Home Reform Law of 1987 prohibit involuntary discharges from nursing homes, Pipal (2012) argues that residents may not be aware of their rights and SNFs may stipulate the possibility of evictions in their admission agreements. Moreover, recent media coverage has highlighted nursing homes' propensity to discharge residents whose Medicare coverage runs out and who are eligible for Medicaid (Siegel Bernard and Pear, 2018). This shows that nursing homes base their discharge decisions at least partly on financial incentives since Medicare reimburses SNFs at higher rates than Medicaid. Overall, the timing of community discharges is largely at the discharge manager's and the resident's discretion. Therefore, we expect that economic incentives may have profound impacts on the length of stay for relatively healthy residents who can return to the community.

\section{A Theoretical Model of Nursing Home Discharges}

In this section, we sketch a theoretical framework that explains how financial incentives of SNFs and residents affect the timing of community discharges. We provide more details when we discuss the empirical version of the model in Section 7. We consider a single SNF

evidence that (permanent) hospitalization discharges do not vary systematically with financial incentives suggesting that strategic hospitalizations are not directly related to our main findings. 
and a single existing resident (the "focal" resident). The SNF maximizes profits and the resident trades off the utility of different care alternatives against the relevant out-of-pocket prices.

Discharges and Effort: In order to increase the probability of a discharge in any given week, the SNF and the resident have to exert costly effort, denoted by $e^{S N F} \geq 0$ and $e^{r e s} \geq 0$, respectively. Motivated by the institutional context and the observed discharged patterns, which we turn to in Section 6, we implicitly rule out "negative" efforts towards delaying the community discharge. The cost of effort includes, for example, the time and resources spent on finding alternative living and care arrangements in the community. We assume that the cost of effort for each agent, $c(e)$, is weakly positive, and strictly increasing and convex in effort. As a result, the SNF and the resident only exert an effort if they prefer a community discharge over staying in the nursing home for an extra period. The SNF and the resident choose their optimal efforts, $e^{S N F, *}(\cdot)$ and $e^{r e s, *}(\cdot)$, as a weakly increasing function of the financial benefits of discharges denoted by FinInc ${ }^{S N F}$ and FinInc ${ }^{\text {res }}$, for nursing homes and residents respectively. As a consequence, the discharge probability $\operatorname{Pr}[D=1]$ also weakly increases in FinInc ${ }^{S N F}$ and FinInc ${ }^{\text {res }}$ :

$$
D(\tau, o c)=\mathbf{1}\left\{\alpha \times e^{S N F, *}\left(\operatorname{FinInc}^{S N F}(\tau, o c)\right)+\beta \times e^{r e s, *}\left(\operatorname{FinInc}^{r e s}(\tau)\right)-\epsilon>0\right\} .
$$

Here, $\alpha \geq 0$ and $\beta \geq 0$ are scalars and capture the effect of financial incentives on nursing home discharges through the nursing home's or the resident's discharge effort. $\tau=P, M$ denotes the focal resident's payer type (private or Medicaid), and oc is the SNF's occupancy rate in beds other than the focal resident's. We assume that the resident's financial discharge incentive and hence her effort are independent of $o c . \quad \epsilon \sim F_{\epsilon}$ captures other factors that determine nursing home discharges, whereby financial incentives only increase discharges in expectation with

$$
\operatorname{Pr}\left[D=1 \mid e^{S N F, *}, e^{r e s, *}\right]=F_{\epsilon}\left(\alpha \times e^{S N F, *}\left(\operatorname{FinInc}^{S N F}(\tau, o c)\right)+\beta \times e^{r e s, *}\left(\operatorname{FinInc}^{r e s}(\tau)\right)\right)
$$

In the case where $\alpha=0$, only the resident's financial incentives matter, and if $\beta=0$ only the SNF's financial incentives determine discharges. If $\alpha, \beta>0$, the relative parameter magnitudes are important in assessing which agent responds more elastically to financial incentives.

Financial Incentives and Discharges: In order to specify how policy interventions affect the length of nursing home stays, we also require a model of the financial discharge incentives. In the case of the nursing home, we consider a dynamic tradeoff. If the focal bed is occupied, 
the nursing home receives a payer type specific flow profit $\Pi^{\tau}$ with $\Pi^{P}>\Pi^{M}>0$. If the resident is discharged, the bed can remain empty, in which case the nursing home forgoes the flow payoff. However, with probability $\Phi(o c)$, the bed is filled with a new resident who may be a private payer or a Medicaid beneficiary. Therefore, the nursing home's optimal discharge effort is determined by the tradeoff between the flow payoff and the option value of drawing a more profitable payer type in the future.

Since private payers are more profitable than Medicaid beneficiaries, a nursing home will not exercise a costly discharge effort if the focal bed is filled with a private payer. Importantly, the refill probability $\Phi(o c)$ is weakly increasing in the occupancy rate in the nursing home's other beds: $\frac{\partial \Phi(o c)}{\partial o c} \geq 0$. Intuitively, the next arriving resident will seek the focal bed with probability one if all other beds are taken. If multiple beds are vacant, however, the probability of filling the focal bed is $<1$. Therefore, the financial incentive as well as the optimal discharge effort are weakly increasing in $o c$ if the focal bed is filled with a Medicaid beneficiary. The occupancy rate in defined over other beds. We assume that the discharge manager, in charge of the focal resident, does not internalize her indirect effect on the occupancy in other beds. In other words, she takes changes in occupancy as given. This assumption simplifies the estimation of the model. We return to the endogenous equilibrium effects of discharge efforts on occupancy in the counterfactual analysis, see Section 8.2.2.

Turning to the resident's effort decision, we consider a static tradeoff. Staying another week yields the utility of nursing home care minus the co-pay. If the resident leaves the nursing home, she obtains the utility of home-based care minus home care payments. Both Medicaid beneficiaries and private payers pay for home care in full, but only private payers pay for nursing home care since the Medicaid co-pay is zero. Conditional on utilities from the two LTC options, private payers have a larger financial discharge incentive and therefore, they exert more discharge effort. The lower discharge effort among Medicaid beneficiaries leads to longer nursing home stays and constitutes patient moral hazard.

We abstract away from strategic free-riding of residents and their relatives on provider effort. We motivate this simplifying assumption by the presence of asymmetric information over the weekly occupancy rate. Building on the limited number of visits by relatives, we assume that they do not observe the weekly occupancy rate and cannot condition their effort on occupancy accordingly. Mechanically, we assume a constant return to resident effort in the empirical analysis which shuts down resident incentives to free-ride. Importantly, we note that free-riding, if present, would work against finding an effect of provider incentives on discharge rates.

Graphical Discussion: We summarize these theoretical predictions in Figure 1, which plots the per period discharge probability by payer type on the vertical axis against the 


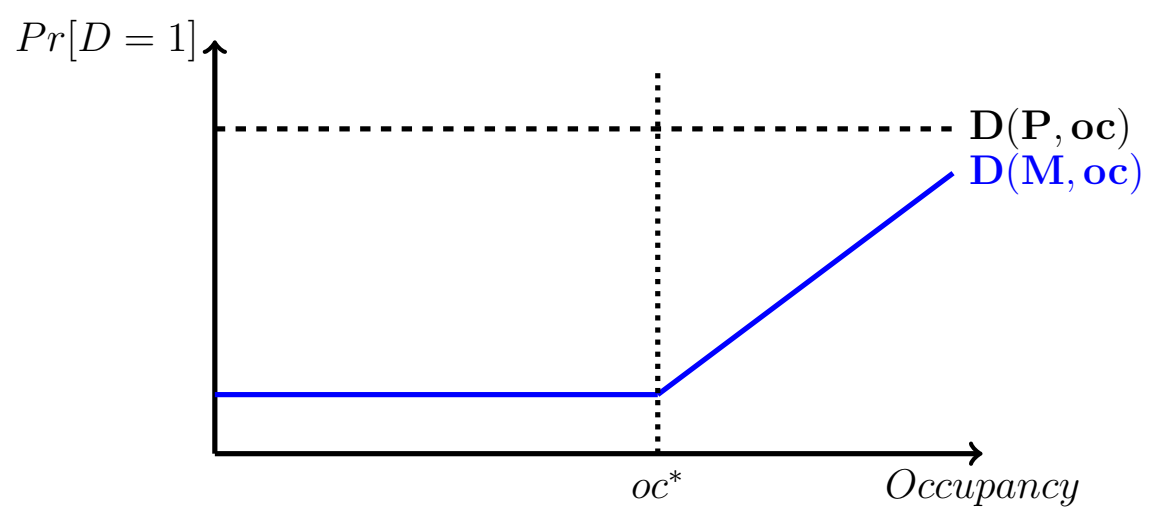

Figure 1: Predicted Discharge Profiles by Payer Type and Across Occupancies

nursing home's occupancy rate on the horizontal axis. The occupancy rate only affects the nursing home's financial incentive. As the nursing home will not exercise effort to discharge a private payer $\left(e^{S N F, *}(P, o c)=0\right.$ for all $\left.o c\right)$ their discharge rates are constant in occupancy as indicated by the horizontal dashed black line. This is not true for Medicaid beneficiaries. At low occupancy rates, nursing homes are not willing to exercise costly effort as the flow payoff exceeds the option value of drawing a private payer (net of the cost of effort) in the future. Intuitively, the refill probability $\Phi(o c)$ is too small, such that the marginal benefit of effort is strictly smaller than the marginal cost of effort. Hence, the nursing home chooses the corner solution of no effort, $e^{S N F, *}(M, o c)=0$ for $o c<o c^{*}$, which explains the horizontal profile in the solid blue line for $o c<o c^{*}$.

Notice however that the discharge probability is smaller for Medicaid beneficiaries at low occupancy rates. This is because private payers exercise a greater discharge effort as they pay the nursing home rate in full: $e^{r e s, *}(P)>e^{r e s, *}(M)$. Hence, the difference in discharge probabilities at low occupancy rates is purely driven by patient incentives, as the nursing home's optimal effort is zero for either payer type at low occupancy rates.

The nursing home's optimal effort decision for Medicaid beneficiaries changes at $o c=o c^{*}$, where the marginal benefit of effort equals the marginal cost of effort at $e^{S N F}=0$, providing an interior solution. The marginal benefit of effort continues to increase in the occupancy rate. Hence, the nursing home raises its optimal effort with increasing $o c$, so it equates the marginal benefit and the marginal cost of effort. The latter increases in effort due to the convex nature of the cost of effort. Hence, we have $e^{S N F, *}(M, o c) \geq 0$ and $\frac{\partial e^{S N F, *(M, o c)}}{\partial o c}>0$ for $o c \geq o c^{*}$. Therefore, the discharge probability of Medicaid beneficiaries increases in the occupancy rate if $o c \geq o c^{*}$, as shown in Figure $1 .{ }^{18}$ We formally derive this relationship under simplifying assumptions in Appendix Section B.

\footnotetext{
${ }^{18}$ We note that the Medicaid discharge rate profile may intersect with the private rate profile at high occupancy rates, depending on the significance of provider incentives.
} 


\section{Data Description}

To investigate the effects of payer type and nursing home occupancy on discharges, we combine resident data from the MDS and Medicaid and Medicare claims data with nursing home characteristics from annual surveys. The MDS contains at least quarterly detailed assessments of SNF residents' health and LTC needs for the universe of residents in Medicaid or Medicare certified nursing homes, about $98 \%$ of all nursing homes. The MDS also provides us with exact dates of admission and discharge, as well as the discharge reason.

We merge the MDS with Medicaid and Medicare claims data at the nursing home stay level. Since only $4 \%$ of nursing home days are covered by private LTC insurance (only $2 \%$ in Pennsylvania), we follow Hackmann (2017) and assume that days not covered by Medicaid or Medicare are paid out-of-pocket. ${ }^{19}$ We return to this simplification in Section 6.2, which can be relaxed using private pay information reported in the MDS. ${ }^{20}$ Building on the claims data, we can then identify the source of payment at each point in time and to specify the timing of transitions from out-of-pocket pay to Medicaid coverage. We merge resident-level data with the On-Line Survey, Certification, and Reporting system (OSCAR), which contains the number of licensed beds, allowing us to calculate weekly occupancy rates. In addition, nursing home surveys from California and Pennsylvania provide us with daily private and Medicaid rates. See Appendix Section C for more details on these data sources.

We use combined data for four states (California, New Jersey, Ohio, and Pennsylvania) for the years 2000 to 2005. Among all individuals who were admitted to a nursing home during this time period, we select those who initially paid for their stay out of pocket, yielding about 1.4 million nursing home stays. We drop Medicare beneficiaries, who require intensive rehabilitative care services and thereby differ from the sample population of interest. We drop residents who are covered by Medicaid from the beginning of their stay to construct a more homogenous sample population with respect to their financial means. Hence, the remaining residents either pay their entire stay out of pocket or become eligible for Medicaid during their stay. We drop about 17,000 resident-stays in which the resident transitions to Medicaid within the first week of the stay because these residents are similar to those who are covered by Medicaid at admission.

Using the admission and discharge dates from the MDS, we convert the data into a long format where each observation corresponds to a resident-week. We drop all resident-week

\footnotetext{
${ }^{19}$ We also note that the average maximum daily benefit of private insurance equals $\$ 109$ in 2000 (the modal benefit was $\$ 100$ ), indicating substantial cost-sharing.

${ }^{20}$ We decided to not use the payer source information from the MDS in our primary analysis due to concerns over potential measurement error Cai et al. (2011). To mediate concerns over MDS measurement error in hospitalization rates, we measure hospitalizations in the Medicare claims data in the health outcome analysis.
} 
observations for nursing homes whose occupancy never exceeds $60 \%$ or at least once exceeds $130 \%$ during the sample period since we are concerned about measurement error in the reported number of licensed beds. This restriction reduces the number of nursing home stays by another 115,000 . To further reduce the role of measurement error in occupancy, we only consider nursing home stays for which the occupancy rate varies between 65 and 100 percent during the entire stay. We also restrict the analysis to nursing homes that accept Medicaid payers. These refinements reduce the number of observations by 350,000. The final sample population consists of about 940,000 nursing home stays and 15.4 million week-stay observations.

These data provide us with the necessary variation in provider and patient discharge incentives. First, we provide evidence for variation in occupancy rates, which drive nursing homes' incentives to discharge Medicaid residents. Figure 2a summarizes the overall variation in occupancy rates over time (weeks) and between nursing homes. The average occupancy rate equals $91 \%$ which translates into 11 empty beds in an average sized nursing home with 120 licensed beds. (See Figure C.2 in Appendix Section C for a histogram of the number of beds.) There is considerable occupancy variation between nursing homes and, more importantly for our analysis, within nursing homes in a given year. Within nursing homes, conditional on nursing home and year fixed effects, we find a standard deviation in occupancy of 3.4 percentage points (about $63 \%$ of the standard deviation in nursing home fixed effects). Figure $2 \mathrm{~b}$ displays this variation graphically.

An important driver of the intertemporal variation in occupancy is the volatility in the number of new admissions. Figure 2c shows the frequency of new admissions divided by total number of beds to translate admissions into changes in occupancy rates. It is evident from this tabulation that the relative number of arrivals can vary substantially from week to week leading to unexpected variation in occupancy. Whether this variation affects providers' discharge incentives in a meaningful way depends in part on the persistence of these occupancy shocks. To assess the persistence, Figure $2 \mathrm{~d}$ displays the impulse response function of occupancy rates to a sudden 3 percentage point increase and decrease in occupancy relative to the sample average. Specifically, we construct an occupancy transition matrix from the data and simulate the occupancy rate profile over time. The response functions indicate that it takes 100 weeks or two years until the occupancy rate reaches its average again. However, it takes only about 25-30 weeks until half of a shock's effects have dissipated, which roughly coincides with the average length of stay of 25.7 weeks in our sample population (indicated by the vertical line in Figure 2d).

The measured persistence of occupancy shocks indicates that nursing homes are likely to take them into account when planning their discharge efforts. In particular, the variation may 


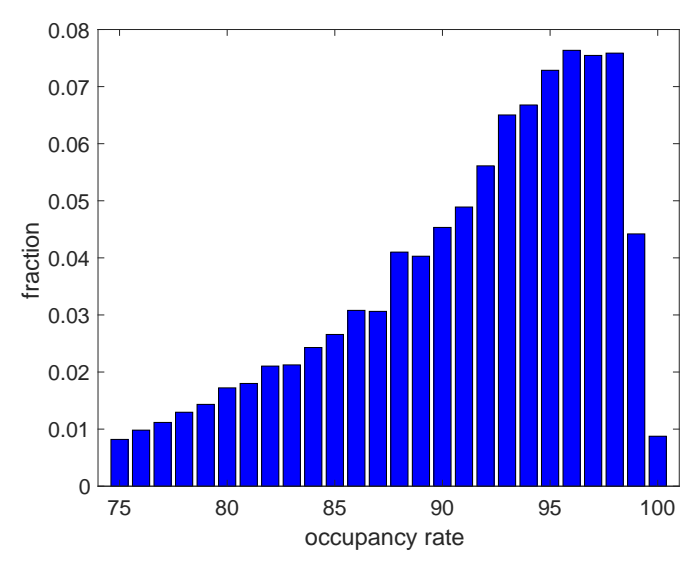

(a) Occupancy Rate Distribution

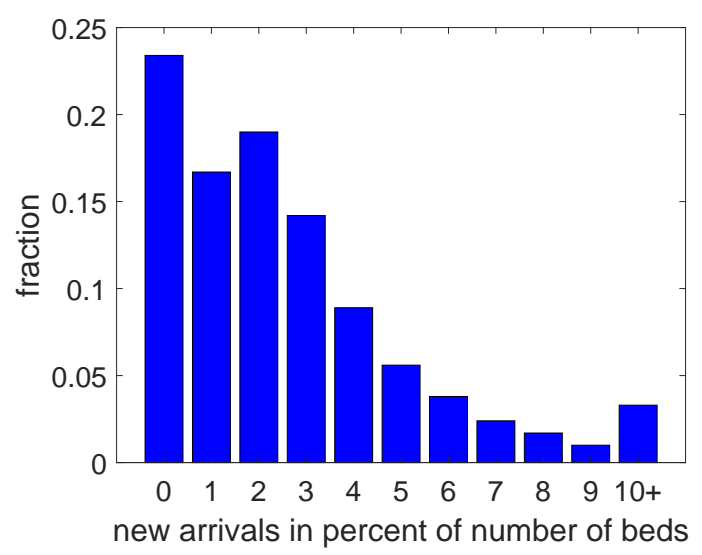

(c) New Arrivals Relative to Number of Beds

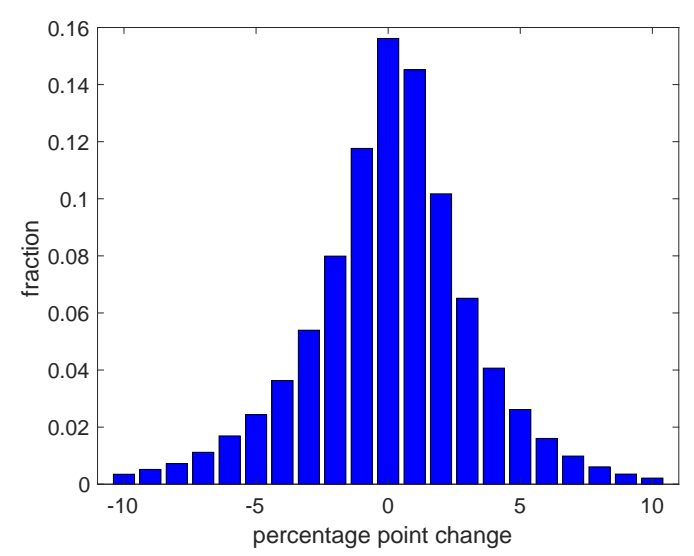

(b) Occupancy Variation Within SNF and Year

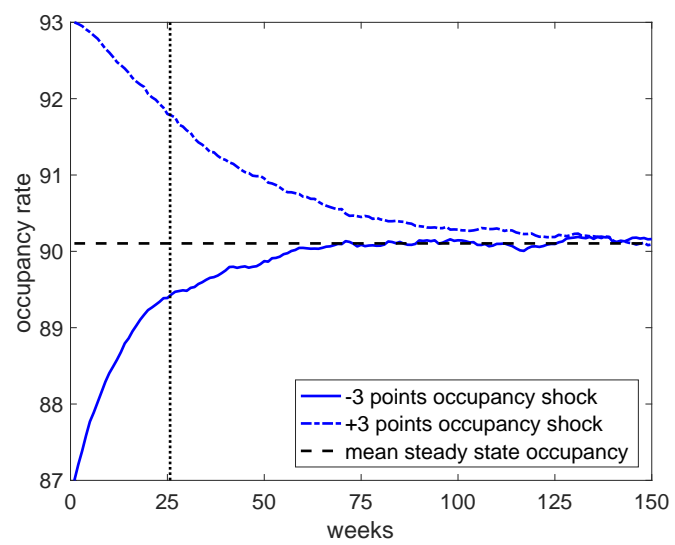

(d) Impulse Response to Change in Occupancy

Notes: Figure 2 a presents occupancy rate variation. Figure $2 b$ shows the residual variation conditional on nursing home year fixed effects. Figure 2c summarizes the frequency of weekly arrivals, divided by the number of licensed beds. The unit of observation for Figures 2a, 2b, and $2 c$ is the nursing home week level. Figure $2 d$ presents two impulse response functions, that document the mean reversion of an initial deviation of \pm 3 percentage points. The vertical line marks the average length of a nursing home stay.

Figure 2: Variation in Occupancy Rates and New Arrivals by SNF and Week

impact the nursing home's community discharge efforts, which is the most common discharge reason in our sample, when measured at the nursing home stay level. About $45 \%$ of the stays end with a community discharge, whereas only $20 \%$ and $15 \%$ of stays, respectively, last until a person passes away or is discharged to a hospital without an anticipated return, see Figure C.1a in Appendix Section C for details. Another $11 \%$ are of stays end with a discharge to a different nursing home and remaining $9 \%$ of stays account for discharges to an assisted living facility and censoring. ${ }^{21}$

\footnotetext{
${ }^{21}$ The relative importance of discharge reasons shifts heavily from home discharges to mortality and censoring, when evaluated at the week-of-stay level. The implicit weighting by length of stay reduces the fraction of home discharges to 11\%, see Figure C.1b in Appendix Section C.
} 
Table 1: Resident-Week-Level Summary Statistics

\begin{tabular}{lccccc}
\hline & \multicolumn{2}{c}{ Private } & & \multicolumn{2}{c}{ Medicaid } \\
\cline { 2 - 3 } \cline { 5 - 6 } & Mean & SD & & Mean & SD \\
\hline Age & 79.05 & $(13.83)$ & & 79.19 & $(12.42)$ \\
Female & 0.62 & $(0.48)$ & & 0.71 & $(0.45$ \\
White & 0.85 & $(0.36)$ & & 0.83 & $(0.38)$ \\
Black & 0.07 & $(0.25)$ & & 0.11 & $(0.31)$ \\
Married & 0.26 & $(0.44)$ & & 0.23 & $(0.42)$ \\
Widowed & 0.47 & $(0.50)$ & & 0.53 & $(0.50)$ \\
Separated/Divorced & 0.08 & $(0.27)$ & & 0.11 & $(0.31)$ \\
Case Mix Index (CMI) & 1.03 & $(0.50)$ & & 1.00 & $(0.44)$ \\
Number of ADL & 10.36 & $(4.80)$ & & 10.70 & $(4.86)$ \\
Clinical Complexity & 0.41 & $(0.49)$ & & 0.35 & $(0.48)$ \\
Depression & 0.34 & $(0.47)$ & & 0.43 & $(0.49)$ \\
Weight Loss & 0.11 & $(0.31)$ & & 0.09 & $(0.29)$ \\
Impaired Cognition & 0.42 & $(0.49)$ & & 0.46 & $(0.50)$ \\
Behavioral Problems & 0.09 & $(0.28)$ & & 0.09 & $(0.29)$ \\
\hline Observations & \multicolumn{3}{c}{$9,693,761$} & & \multicolumn{2}{c}{$5,711,288$} \\
\hline
\end{tabular}

Notes: Data are from the MDS 2000 to 2005. The table presents summary statistics by payer source at the week of stay level. The resident's health status is decreasing in each health measure. The CMI is a summary measure of long term care needs, calculated based on methodology 5.01, and normalized to 1. The remaining health measures are direct inputs to the CMI formula and provide more granular information on cognitive and physical disabilities.

Second, we compare Medicaid beneficiaries with residents who pay out of pocket. Table 1 shows resident-week-level summary statistics for our estimation sample, split by payer type in the given week of the stay. Overall, the payer types are remarkably similar in terms of socio-demographics and their health profiles. However, Medicaid beneficiaries are slightly healthier, based on the CMI, more likely to be black and less likely to reside in a for-profit nursing home. Moreover, Medicaid beneficiaries are substantially more likely to be female and widowed. These differences may point to potential differences in access to informal care givers if, for example, women provide informal long term care for their husbands. To investigate potential differences in access to community-based care further, we supplement Table 1 with data from from the National Long Term Care Survey (NLTCS) and the Health and Retirement Study (HRS). The HRS data indicate that access to informal care givers is similar between private payers and Medicaid beneficiaries. With respect to financial outcomes, the NLTCS data point to higher home ownership rates among private payers when compared to residents who transition into Medicaid but the difference is not statistically different from zero, see Appendix Section C. We view these differences between 
Medicaid beneficiaries and private payers as relatively minor.

\section{$5 \quad$ Empirical Strategy}

We exploit within nursing home and year variation in patient's and provider's financial incentives and model their effect on the weekly discharge rate (hazard rate). To ease the computational burden of the large number of observations and fixed effects, we estimate a series of linear probability models. We can flexibly control for duration dependence via week-of-stay fixed effects and assume that the residuals are i.i.d. over time.

Specifically, we estimate the following reduced form regression model for equation (1), which expresses financial incentives as flexible functions of occupancy and payer type:

$$
Y_{i j s t}=\sum_{k=65}^{100} \gamma^{k} o c c_{j t-1}^{k}+\sum_{k=65}^{100} \delta^{k} o c c_{j t-1}^{k} M_{c a i d} d_{i s}+\alpha_{j y}+\alpha_{c}+\alpha_{s}+X_{i}^{\prime} \beta+\epsilon_{i j s t} .
$$

Here $Y_{i j s t}$ denotes an indicator variable that equals one if resident $i$ in nursing home $j$ was discharged to the community in stay week $s$ and calendar week $t$. occ $c_{j t}^{k}$ is an indicator variable that turns on if the (rounded) occupancy rate equals $k=65, \ldots, 100$ percent in nursing home $j$ in calendar week $t$. Mcaid $_{i s}$ is an indicator variable for resident $i$ being covered by Medicaid in week $s$ of her stay. ${ }^{22}$

The coefficients of interest are $\gamma^{k}$ and $\gamma^{k}+\delta^{k}$, which can be interpreted as the effect of occupancy on weekly discharge probabilities for private payers and Medicaid beneficiaries, respectively. We are particularly interested in the differences between payer types captured by $\delta^{k}$. These estimates are conditional on facility-year fixed effects $\alpha_{j y}$, calendar month fixed effects $\alpha_{c}$, week-of-stay fixed effects $\alpha_{s}$, and health status at admission $X_{i}$.

Occupancy may be endogenous to discharges for several reasons. First, there is a mechanical reverse relationship between the own discharge process and the occupancy rate. To address this issue, we use a leave-one-out measure for the occupancy rate. Specifically, we explore occupancy rate variation in other beds. To implement this strategy, we use the lagged occupancy rate, which only varies in other beds, once we exclude the first week of the stay. To see this, note that an individual resident only affects the facility-level occupancy rate in the weeks when she is admitted and discharged. By dropping the first week of each stay and using the lagged occupancy rate, we remove the variation in the last week of each nursing home stay that is partly due to the resident's own discharge.

Second, to control for potential drivers of discharges that may be correlated with the occupancy rate and the resident's payer type, we add week-of-stay fixed effects $\alpha_{s}$ in regres-

\footnotetext{
${ }^{22}$ Given the Medicaid eligibility rules that depend on asset spend-down (see Section 2), the Medicaid indicator switches from 0 to 1 at most once during a resident's stay.
} 
sion (2). These effects control for unobserved differences in health types that are important for the remaining length of stay. For example, the discharge probability in a given week is considerably lower for residents that have already spent six months in the nursing home compared to residents who were admitted within the previous two weeks. In other words, the week of stay fixed effects flexibly control for duration dependence in the discharge profiles. We also control for observable health measures at admission contained in $X_{i}$ to construct a homogenous comparison population. ${ }^{23}$ In addition, we control for calendar month fixed effects, $\alpha_{c}$, to account for seasonal variation in discharges. Finally, we control for facility-year fixed effects captured by $\alpha_{j y}$ in regression (2). Any unobserved effects that may systematically impact discharge rates, such as nursing homes' management decisions, prices, and quality, are absorbed in this fixed effect. Hence, we only explore variation in occupancy within nursing homes in a given year. We assume that the residuals are i.i.d. over time and therefore uncorrelated with the right-hand side control measures, that are all history-based.

Given our rich set of nursing home-year fixed effects and week of stay fixed effects, we compare residents in the same nursing home and year and the same week of their stay. We therefore attribute remaining differences in discharge rates between payer types and across occupancies to financial incentives.

\section{Empirical Discharge and Health Patterns}

In this section, we provide reduced-form evidence for financial patient and provider incentives driving nursing home discharges, i.e. we test the implications of the theoretical model presented in Section 3.

\subsection{Home Discharge Patterns by Occupancy and Payer Type}

Figure 3 is the empirical analogue to Figure 1 and shows the estimated effects of occupancy and payer type on the home discharge rate along with the $90 \%$ confidence intervals. The depicted estimates correspond to the mean-adjusted coefficients $\hat{\gamma}^{k}$ for private payers and $\hat{\gamma}^{k}+\hat{\delta}^{k}$ for Medicaid beneficiaries, respectively. These estimates are conditional on facilityyear, month, and week of stay fixed effects and resident characteristics including health at admission and therefore isolate the effect of occupancies on payer type specific discharge rates from potential selection due to length of stay or resident's health.

The figure provides two important insights. First, private payers have higher discharge rates across the entire range of occupancies. On average, private payers have a $2 \%$ chance of being discharged to the community in a given week. In contrast, below $80 \%$ occupancy,

\footnotetext{
${ }^{23}$ These health measures include the individual case mix index, as well as the predicted length of stay. We construct the latter by regressing length of stay on a rich set of disability and health measures and obtaining the predicted outcome for each resident.
} 


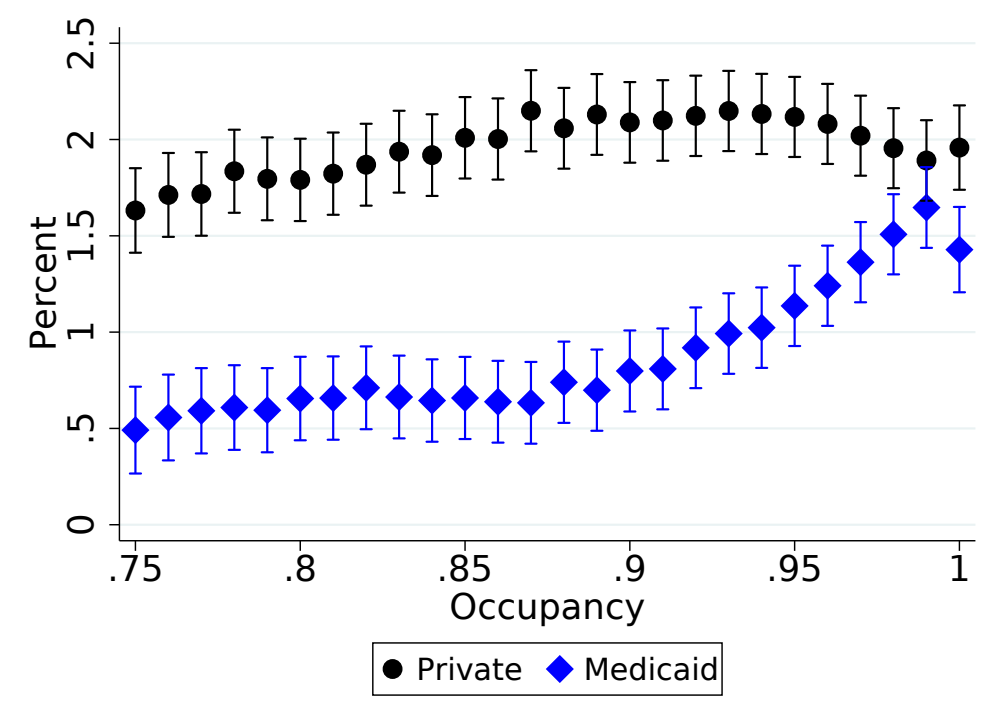

Notes: This figure plots the estimated coefficients $\hat{\gamma}^{k}$ (private) and $\hat{\gamma}^{k}+\hat{\delta}^{k}$ (Medicaid) from regression (2) for the dependent variable "home discharge" across occupancy rates $k$. The coefficients are adjusted to match observed mean discharge rates by payer type. The vertical bars indicate $90 \%$ confidence intervals.

Figure 3: Home Discharge Rates by Payer Type and Occupancy

the discharge rate among Medicaid residents equals only $0.9 \%$, a difference of more than 1 percentage point, see also the first row of Table 2. This observation is consistent with differences in resident cost-sharing, as discussed in Section 3. Since SNFs do not have a financial incentive to discharge residents of either payer type at low occupancies, the estimated difference in discharge rates suggests that resident incentives affect the length of stay. To put this difference into perspective, we simulate the length of stay for private and Medicaid beneficiaries, taking other discharge reasons into account. We return to the details of this calculation in Section 8. Ignoring changes at higher occupancies, we find that the 1.1 percentage point difference in discharge rates raises the length of stay of Medicaid beneficiaries by $36 \%$ when compared to private payers. Considering the $100 \%$ price reduction from private pay to Medicaid coverage, this suggests a price elasticity of demand of 0.36 , see column 5 of Table 2, which is slightly larger than seminal estimates from the RAND and the Oregon experiments, which center around 0.2, see Manning et al. (1987), Finkelstein et al. (2012), as well as Shigeoka (2014) for elderly people. However, the elasticity is significantly smaller than estimates of the price elasticity of substitution between nursing homes, see Ching, Hayashi, and Wang (2015) and Hackmann (2017).

Second, above $89 \%$ occupancy, Medicaid home discharge rates increase, reaching $2 \%$ as occupancy approaches $100 \% .{ }^{24}$ Interpreted through the lens of the theoretical model, the

\footnotetext{
${ }^{24}$ With respect to our estimates at $100 \%$ occupancy, we note that these are likely contaminated by
} 
Table 2: Discharge Rates and Price Elasticities by Patient and Provider Incentives

\begin{tabular}{lcccccc}
\hline & $\begin{array}{c}D_{\text {low }}^{P}-D_{\text {low }}^{M} \\
(1)\end{array}$ & $\begin{array}{c}D_{\text {high }}^{P}-D_{\text {high }}^{M} \\
(2)\end{array}$ & $\begin{array}{c}(2)-(1) \\
(3)\end{array}$ & $\begin{array}{c}\text { oc* } \\
(4)\end{array}$ & $\begin{array}{c}\epsilon^{\text {res }} \\
(5)\end{array}$ & $\begin{array}{c}\epsilon^{S N F} \\
(6)\end{array}$ \\
\hline Baseline & $1.15 \%$ & $0.89 \%$ & $-0.26 \%$ & $89 \%$ & 0.36 & \\
Low Patient Incentives & $0.74 \%$ & $0.42 \%$ & $-0.32 \%$ & $89 \%$ & 0.37 & \\
High Patient Incentives & $1.00 \%$ & $0.37 \%$ & $-0.63 \%$ & $70 \%$ & 0.37 & \\
Forward-looking Patients & $1.59 \%$ & $1.29 \%$ & $-0.23 \%$ & $89 \%$ & 0.39 & \\
\hline Low Provider Incentives & $0.62 \%$ & $0.38 \%$ & $-0.25 \%$ & $89 \%$ & & 1.18 \\
High Provider Incentives & $0.94 \%$ & $0.17 \%$ & $-0.77 \%$ & $70 \%$ & & 1.18 \\
Admission Occupancy & $0.71 \%$ & $0.41 \%$ & $-0.30 \%$ & $91 \%$ & & \\
\hline
\end{tabular}

Notes: This table summarizes evidence on patient and provider incentives. Column (1) shows differences in weekly discharge rates between private and Medicaid beneficiaries at occupancies below 80\%. Column (2) provides analogues evidence for occupancies above 90\%. Column (4) displays the estimated kink point in the Medicaid discharge profile following Hansen (2017). Columns (5) and (6) display implied patient and provider elasticities with respect to changes in financial incentives.

estimated kink at $89 \%$ occupancy, see the fourth column of Table 2, marks the point at which the nursing home starts to exercise a positive discharge effort. ${ }^{25}$ At lower occupancies, nursing homes benefit from extended Medicaid stays, to the extent that Medicaid rates exceed the marginal cost of care. At higher occupancies, this incentive is muted because nursing homes prefer to occupy their scarce beds with more profitable private payers. In contrast, private payers' home discharge rates vary little (between $1.7 \%$ and $2.1 \%$ ) and not systematically with occupancy. Consistent with the theoretical predictions, these findings suggest that provider incentives affect the length of stay as well. Unfortunately, we cannot translate the change in Medicaid discharges rates into a supply side elasticity, as we require a supply side model to pin down the financial incentives. We will return to this calculation in Section 8.

In Appendix Section D.2, we show that discharges to a hospital, another nursing home or due to the resident's death vary with occupancy to a much smaller extent. In fact, as we discuss in greater detail below, changes along these discharge margins predominantly reflect changes in the health composition of residents as a result of endogenous changes in home discharges. We therefore conclude that financial patient and provider incentives operate mostly through the home discharge margin.

measurement error, which biases the estimates towards the average discharge probability across occupancy rates. This explains the modest reverse in the discharge pattern.

${ }^{25}$ The estimation strategy for the kink point follows Hansen (2017), see the Appendix Section D.1 for details. 


\subsection{Patient Incentives and Discharges}

Following the theoretical predictions, differences in discharge rates between payer types at low occupancies indicate that patient financial incentives affect the length of nursing home stays. In this section, we briefly summarize a series of robustness exercises that corroborate this interpretation. We are primarily concerned about two sources of bias and organize our discussion around theses threats to identification.

\subsubsection{Unobserved Patient Heterogeneity}

A potential concern is that unobserved differences between Medicaid beneficiaries and private payers - for example differences in health profiles - may at least partially explain the observed differences in discharge rates at low occupancies. While we control for a rich set of health characteristics at admission, including the predicted length of stay based on observed health measures, unobserved differences in health profiles could explain differences in discharge rates. To alleviate this concern, we note that we find qualitatively very similar discharge profiles in a more homogenous and relatively healthy patient population with minimal LTC needs, see Figure D.2 in Appendix Section D.3. Furthermore, as discussed in Section 6.4, financial incentives lead to longer stays (on net) among relatively healthy Medicaid beneficiaries. Therefore, Medicaid beneficiaries are (ex post) advantageously selected, providing evidence against this concern. Consistent with this observation, we find that conditional on health at admission and week of stay fixed effects, Medicaid residents appear healthier as evidenced by a lower CMI, see Figure D.3 in Appendix Section D.4.

Alternatively, private payers may have higher discharge rates because they have better access to home health care as they are wealthier than their peers who transitioned into Medicaid. As a first step towards constructing a sample population that is homogenous with respect to financial and medical characteristics, we focus on residents who pay out-of-pocket at the beginning of their stay. Data from the NLTCS suggest that differences in income and wealth become statistically insignificant once we make this refinement, see Appendix Section $\mathrm{C}$ for details. Furthermore, as mentioned above, the differences in discharge rates are also visible among relatively healthy seniors who do not require home-based health care and for whom living in the community is therefore more affordable.

Robustness: We undertake several additional attempts to mitigate concerns over the role of differences in financial means or health profiles between private payers and Medicaid beneficiaries. First, we exploit rich cross-sectional variation in private rates between nursing homes to test for differences in patient incentives between high and low price nursing homes. Using price data from Pennsylvania and California, we divide the sample of nursing homes into high and low patient incentive facilities, depending on whether the private rate exceeds 
or falls short of the median price in the respective state. We then re-estimate equation (2) for each subsample. Consistent with the predictions from the theoretical model, we find smaller differences between private and Medicaid discharge rates at low occupancy rates when private rates are smaller, see the column (1) in the second and third rows of Table 2. Using a simulation model, we conclude that the difference-in-differences of discharge rates $(0.63 \%-0.32 \%=0.31 \%$, see column (3) of rows 2 and 3$)$ translates into a slightly larger price elasticity of 0.37 , when considering the average difference in private rates between the two samples of $43 \%$. The similarity in the price elasticities lends further support to our baseline findings on patient incentives. This finding also alleviates concerns that partial insurance provided by private LTC insurance or partial cost-sharing in medically needy programs may add bias to the baseline patient incentive estimates. ${ }^{26}$

To explore the relationship between discharge rates and the cross-sectional price variation at a more granular level, we also estimate the following variant of equation (2):

$$
\begin{aligned}
Y_{i j s t} & =\mathbf{1}\left\{o c_{j t-1}<80 \%\right\} \text { Mcaid }_{i s} \times \sum_{\tau=1}^{10} \delta_{\tau}^{l} \mathbf{1}\left\{r_{j t}^{P} \in P I^{\tau}\right\} \\
& +\mathbf{1}\left\{o c_{j t-1}>90 \%\right\} \text { Mcaid }_{i s} \times \sum_{\tau=1}^{10} \delta_{\tau}^{h} \mathbf{1}\left\{r_{j t}^{P} \in P I^{\tau}\right\}+\delta \text { Mcaid }_{i s} \\
& +\sum_{k=65}^{100} \gamma^{k} o c_{j t-1}^{k}+\alpha_{j y}+\alpha_{c}+\alpha_{s}+X_{i}^{\prime} \beta+\epsilon_{i j s t}
\end{aligned}
$$

where the first two rows replace $\sum_{k=65}^{100} \delta^{k} o c c_{j t-1}^{k} M c a i d_{i s}$ from equation (2). Specifically, we aggregate occupancy rates into low (less than 80\%) and high rates (more than 90\%) to reduce the number of parameters. We then interact the occupancy groups with series of indicator variables, $\mathbf{1}\left\{r_{j t}^{P} \in P I^{\tau}\right\}$, that turn on if the nursing home's private rate falls into the relevant price decile. The key parameters of interest are $\delta_{\tau}^{l}+\delta$, which govern differences in discharge rates between Medicaid beneficiaries and private payers at low occupancy rates for different private rate deciles. Figure 4a presents the point estimates along with $90 \%$ confidence intervals. The downward sloping relationships suggests that differences in discharge rates at low occupancy rates are more pronounced in nursing homes with higher private rates, which corroborates the baseline evidence on patient incentives.

In addition to exploring cross-sectional price variation, we also revisit the analysis in a more refined sample population to mitigate concerns over the role of unobserved patient

\footnotetext{
${ }^{26}$ To see this, notice that patients pay a top-up in both cases and are thereby exposed to the full variation in private rates exploited in this robustness exercise. For example, private LTC insurance typically pays an average maximum daily benefit of only $\$ 100$ and asks patients to pay the full difference to the private rate out-of-pocket.
} 


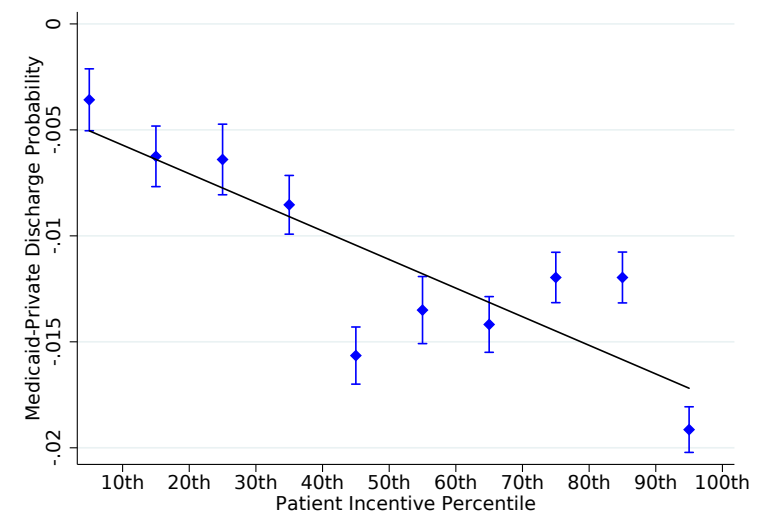

(a) Patient Incentives

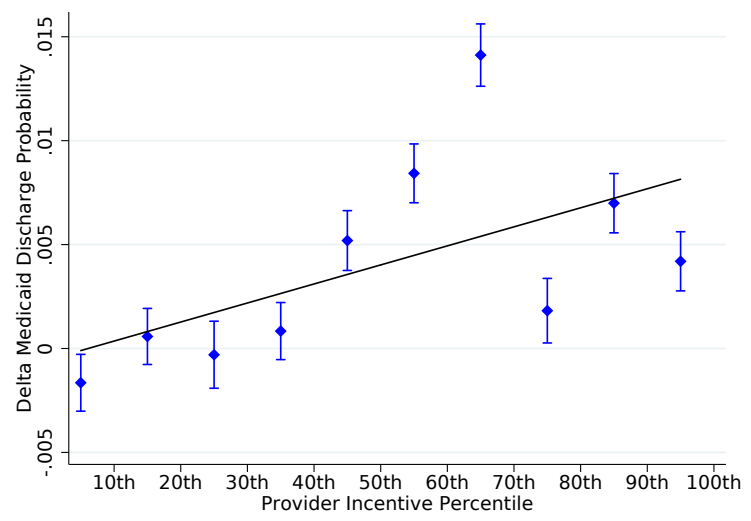

(b) Provider Incentives

Notes: Figure 4 a summarizes mean differences in private and Medicaid discharge rate at occupancy rates below $80 \%$ across nursing homes in different private percentiles. Figure $4 b$ summarizes mean differences Medicaid discharge rates at occupancy rates exceeding $90 \%$ and occupancies below $80 \%$ across nursing homes different private over Medicaid rate markup percentiles. The vertical bars indicate $90 \%$ confidence intervals.

Figure 4: Home Discharge Rates and Health Outcomes by Payer Type and Occupancy

heterogeneity. Specifically, we consider a propensity weighting approach, where we predict Medicaid transitions using the rich demographic information in the MDS, including the zip code of the former residence, the educational attainment, as well as gender, age, and race. This approach balances the covariates of residents who transition into Medicaid and those who keep paying out-of-pocket and delivers results that are similar to our main findings in Figure 3, see Appendix Section D.5. In an additional robustness exercise, we focus on Medicaid applicants. Not all Medicaid applicants are granted Medicaid, providing us with payer type variation among a pool of residents who appear sufficiently poor to consider applying for benefits. Again we find very similar albeit noisier differences in discharge rates between payer types in this smaller sample population, see Appendix Section D.6.

Finally, and complementary to the former two robustness approaches, we also exploit differences in Medicaid eligibility rules between New Jersey, Ohio, and Pennsylvania in a border analysis. Using state of residence as an instrument for Medicaid eligibility, we find further evidence for reduced discharge rates among Medicaid beneficiaries, see Appendix Section D.7. Furthermore, we also investigate bunching in the length of stay of Medicare beneficiaries, who are excluded from our main analysis. Medicare beneficiaries face a daily co-pay of about $\$ 150$ starting on their 21st day of coverage in the respective reimbursement episode. We find clear graphical evidence around the onset of co-pay, which again corroborates the point that nursing home residents adjust their length of stay to financial incentives, see Figure D.5.

Overall, these additional exercises all confirm our baseline finding that financial patient 
incentives affect the length of nursing home stays. Therefore, we abstract away from unobserved patient heterogeneity in the structural analysis and postpone the discussion of how this assumption affects our main findings to Section 8.3.

\subsubsection{Forward Looking Patients}

A second concern is that patients are forward looking and anticipate a transition from private pay to Medicaid coverage as they deplete their assets. If so, private payers with lower assets at admission may lower their discharge efforts (lowering the discharge probability) to benefit from Medicaid coverage in the near future. This would bias the estimated private discharge probability downward and thereby understate the role of patient incentives.

To investigate this possibility, we conservatively drop all private pay (weekly) observations among seniors who eventually transition to Medicaid during their stay. By construction, seniors have not been discharged in these weeks, otherwise they could not transition to Medicaid at a later point of their stay. We conservatively attribute the implied $0 \%$ discharge rate in these weeks entirely to forward looking patient behavior. Dropping these observations raises the private discharge probability and the difference between private and Medicaid discharge rates by about 0.4 percentage points, see the fourth row of Table 2 . The larger difference translates into a slightly larger patient elasticity of 0.4. Given the relatively small effect on the implied patient elasticity, we abstract away from forward looking behavior in the structural analysis and return to this point in the robustness discussion of our counterfactual findings.

\subsection{Provider Financial Incentives and Discharges}

We explain the observed increase in Medicaid discharge rates at high occupancy rates with an increasing option value of an empty bed. To corroborate this interpretation we perform several additional tests and robustness exercises.

\subsubsection{Bed Refill Probability}

First, we provide direct evidence on the refill probability, which determines the option value of an empty bed in our framework. Specifically, we combine the observed number of vacant beds and realized admissions to measure the weekly probability that an empty bed is refilled.

To this end, we consider a nursing home with $a \geq 0$ newly arriving seniors per week. We assume that arriving seniors are randomly assigned to $v$ vacant beds. If $a>v$, demand exceeds capacity and the nursing home must turn away $a-v$ of the newly arriving seniors. 
The probability that a focal bed remains empty in a given week equals:

$$
\operatorname{Pr}[\text { Not Refilled }]= \begin{cases}\frac{v-1}{v} \times \frac{v-2}{v-1} \times \cdots \times \frac{v-a}{v-a+1}=\frac{v-a}{v} & \text { if } a<v \\ 0 & \text { otherwise }\end{cases}
$$

Hence, the probability the bed is refilled is simply:

$$
\Phi=\operatorname{Pr}[\text { Refilled }]=1-\operatorname{Pr}[\text { Not Refilled }]=1-\max \left\{\frac{v-a}{v}, 0\right\} .
$$

We note that censoring in admissions, induced by rationing, may bias the number of observed newly arriving seniors $a$ downward. We are, however, less concerned about a downward bias in the refill probability since $\Phi=1$ for $k \geq v$. In other words, there is no downward bias in $\Phi$ as long as we observe $a=v$ whenever $a \geq v$.

We measure $\Phi$ at the nursing home week level, and construct its conditional mean by weekly occupancy. We find a highly convex relationship between the refill rate and the occupancy rate. The refill probability increases only from $10 \%$ to $18 \%$ between $75 \%$ and $90 \%$ occupancy. However, between $90 \%$ and $100 \%$ occupancy, the refill rate increases drastically from $18 \%$ to $60 \%$, see Figure D.6 in Appendix Section D.9 for details. We also note that $78 \%$ of newly-admitted (non-Medicare) residents pay out-of-pocket at the beginning of their stay. Combined with the high refill probability at high occupancy rates, this provides nursing homes with a strong incentive to discharge Medicaid beneficiaries at high occupancies as observed in Figure 3.

\subsubsection{Differences in Provider Incentives}

Second, we exploit the rich cross-sectional variation in private and Medicaid rates between nursing homes in Pennsylvania and California to test for differences in provider incentives. For this exercise, we use the private rate markup over the Medicaid rate, $\mu_{j t}=\left(r_{j t}^{P}-r_{j t}^{M}\right) / r_{j t}^{M}$ as a proxy for provider incentives and divide the sample into high and low incentive nursing homes depending on whether their markup exceeds or falls short of the median markup in the respective state. We then re-estimate equation (2) for each subsample. Consistent with the predictions from the theoretical model, we find larger declines in the difference between

private and Medicaid rates at high incentive nursing homes when going from low to high occupancy. The decline equals 0.77 percentage points for high incentive homes but only 0.25 percentage points for low incentive homes, see column (3) of rows 5 and 6 in Table 2. We also estimate a lower kink point for high incentive nursing homes at about $70 \%$ occupancy, again consistent with the theoretical predictions. Unfortunately, we can cannot map the differences in discharge profiles into a provider price elasticity without specifying the 
underlying financial incentives, which we turn to in the structural estimation. Nevertheless, to provide a first estimate, we compare the different Medicaid discharge profiles between low and high incentive nursing homes and simulate differences in the length of stay. Dividing the difference by the average private rate difference of $24 \%$, we find a supply side elasticity of 1.18. ${ }^{27}$ This estimate exceeds the patient elasticities by a factor of 3 providing first suggestive evidence that providers respond more elastically to financial incentives.

We also explore the relationship between provider incentives and discharge rates by using the cross-sectional price variation at a more granular level and by estimating a variant of equation (3). Shifting the focus to provider incentives, we replace $1\left\{r_{j t}^{P} \in P I^{\tau}\right\}$ by $\mathbb{1}\left\{\mu_{j t} \in M U^{\tau}\right\}$, which turns on if the nursing home's private rate markup falls into the relevant markup decile. The key parameters of interest are now $\delta_{\tau}^{h}-\delta_{\tau}^{l}$, which govern differences in Medicaid discharge rates at high and low occupancies. Figure 4b presents the point estimates along with $90 \%$ confidence intervals. The upward sloping relationship suggests that differences in Medicaid discharge rates between high and low occupancy are larger in nursing homes with higher incentives, which corroborates the baseline evidence on provider incentives.

\subsubsection{Selective Admissions vs. Discharges}

An alternative explanation for the increase in Medicaid discharge rates at high occupancy rates is that providers cream-skim different patients at admission as they operate under higher occupancy rates. We note that we focus exclusively on seniors who pay out-ofpocket at the beginning of their stay and control for rich health characteristics at admission. Nevertheless, it could be that, at high occupancy rates, providers disproportionately creamskim unobservably healthy seniors with shorter lengths of stay among those seniors who eventually transition to Medicaid. To control for potential cream-skimming at admission, we add a series of occupancy fixed effects, evaluated at admission, and interacted with payer type in the current week of the stay as further control variables to equation (2).

We summarize the revised discharge profiles in the last row of Table 2. We find a slightly smaller difference in discharge profiles at low occupancy rates pointing to a smaller patient elasticity. Importantly, we find a very similar convergence in discharge profiles as the occupancy increases when compared to the baseline estimates. The estimated kink point in the Medicaid discharge profile increases slightly to an occupancy rate of $91 \%$. These estimates suggest that provider discharge incentives are robust to controlling for potential cream-skimming at admission. We return to this point in the structural analysis (Section 8).

\footnotetext{
${ }^{27}$ The Medicaid rates are almost identical on average between the samples, $\$ 157$ vs. $\$ 160$, which is why we focus on the private rate differential when estimating the elasticity.
} 


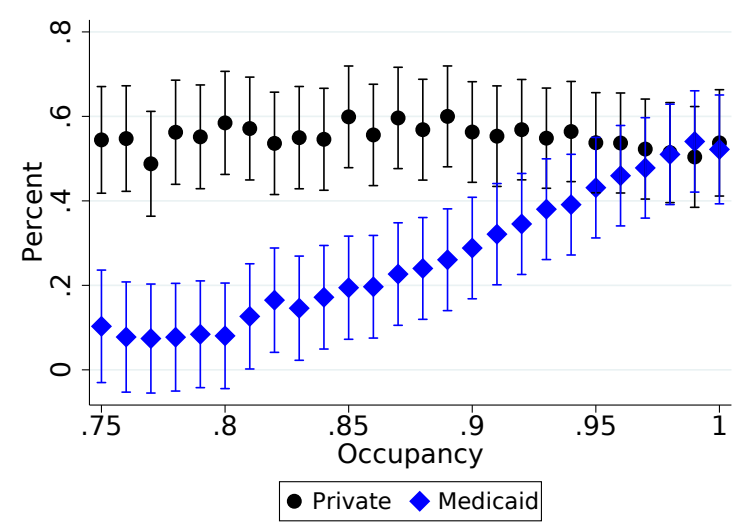

(a) Home Discharges Without Home Health Care

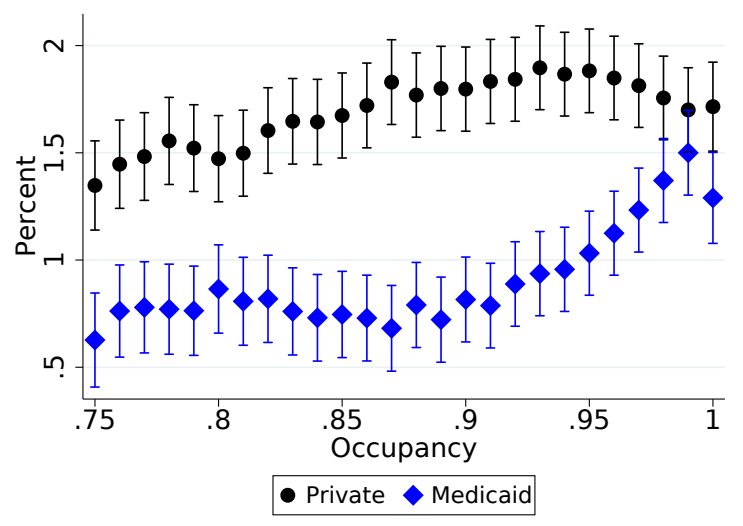

(b) Home Discharges With Home Health Care

Notes: See notes for Figure 3. Figure 5a and Figure $5 b$ display analogues results for discharge to the community without requiring home health care or with home health care, respectively. The vertical bars indicate $90 \%$ confidence intervals.

Figure 5: Home Discharge Rates and Health Outcomes by Payer Type and Occupancy

\subsection{Marginal Residents and Health Benefits From Longer Stays}

We now turn to the effects of longer nursing home stays on patient health. To put our findings into perspective, we start with an analysis of the marginal Medicaid beneficiary population before turning to evidence on one-year hospitalization and mortality rates, as well as health profiles at the time of discharge.

Marginal residents: To gain a better understanding of the marginal Medicaid residents who are discharged at high occupancies, we take advantage of more granular information on the discharge code. Specifically, the MDS divides home discharges into cases that require home health follow up care and cases that do not. Figure 5a documents the effects of occupancy and payer type on community discharges that do not require home health care. Here, we see relatively larger changes in Medicaid discharge rates than in Figure 3. Discharge rates increase about sixfold between $75 \%$ and 100\% occupancy, from a weekly discharge rate of $0.1 \%$ to about $0.6 \%$. In contrast, discharge rates among private payers do not vary with occupancy and equal about $0.6 \%$ throughout the occupancy range. Figure $5 \mathrm{~b}$ presents the results for community discharges that require some home health care. Here the profiles are similar to the patterns for community discharges overall.

These results indicate that the residents at the margin of being discharged home are relatively healthy and often do not require home health care. Hence, longer nursing home stays among these Medicaid beneficiaries may contribute towards LTC overspending. Interestingly, the discharge rates for healthy Medicaid beneficiaries in Figure 5a exhibit a kink point 
at a lower occupancy rate of only $80 \%$. Nursing homes therefore seem to weigh financial benefits and the LTC needs of their residents when deciding on their discharge effort.

We revisit the health composition of marginal residents in additional analyses, in which we restrict the sample population to residents who do not require SNF care on clinical grounds. Following Mor et al. (2007), we restrict the sample to seniors with very low care needs and high prospects to return to the community. Within this subpopulation, we find higher average discharge rates, larger level differences between Medicaid and private payers at low occupancy rates, and larger increases in discharge rates for Medicaid beneficiaries as the occupancy rate increases from about 87\% to 100\%, see Figure D.2 of Appendix Section D.3. These findings corroborate the view that marginal residents appear to be relatively healthy and support the concern that longer nursing home stays may contribute to overspending.

The fact that marginal residents are relatively healthy also suggests that financial incentives lead (ex post) to an advantageous selection of Medicaid beneficiaries. If financial incentives lead to longer nursing home stays among healthy Medicaid beneficiaries, then conditional on health at admission, Medicaid beneficiaries in nursing homes are advantageously selected when compared to residents who pay out-of-pocket. This prediction is supported by lower weekly mortality rates for Medicaid beneficiaries, presented in the middle right graph of Figure D.1 in Appendix Section D.2. Interestingly, the difference in mortality decreases as the occupancy rate increases. Increasing financial provider incentives reduces the length of stay for healthy marginal Medicaid beneficiaries and therefore mutes the ex post advantageous selection. These hypotheses are further supported by the evidence on hospital discharge rates presented in the top right graph of Figure D.1.

Health Outcomes: Next we investigate the health returns to longer nursing home stays among marginal Medicaid beneficiaries. To this end, we exploit the occupancy rate as a source of plausibly exogenous variation in the remaining length of stay. We use the oneyear hospitalization rate, which is commonly viewed as a negative health outcome, and the one-year mortality rate as dependent variables in regression (2). Our estimates point to a statistically significant reduction in the one-year hospitalization rates among Medicaid beneficiaries at high occupancies (>90\%), which is when nursing homes exercise effort to shorten Medicaid stays, see Figure D.8 in Appendix Section D.10. In contrast, we find no conclusive evidence for mortality rates.

Complementing the evidence on hospitalizations and mortality, we explore changes in seniors' overall health status and the amount of ADL limitations they suffer at the time of discharge. If longer SNF stays lead to better health outcomes, we expect that Medicaid residents are healthier than private payers at the time of discharge. We also expect a decline in Medicaid residents' health above $90 \%$ occupancy when their discharge rate increases. As 
shown in Figure D.9 in Appendix Section D.10, we can reject both predictions.

Overall, we conclude that marginal Medicaid beneficiaries appear to be relatively healthy. We also find no evidence that longer stays lead to improved health outcomes suggesting that longer nursing home stays (on this margin) likely constitute over-utilization of nursing home care. These observations are consistent with the findings in Doyle, Graves, and Gruber (2017) and Jin, Lee, and Lu (2018) who argue that SNF use after hospitalizations is likely harmful and wasteful.

\section{Structural Model of Discharges}

In the previous section, we documented that both provider and patient incentives affect nursing home discharge rates. In order to give the reduced form parameters a structural interpretation, to compare the significance of resident and provider incentives, and to evaluate counterfactual policy experiments, we now turn to a structural model of nursing home discharges.

\subsection{The Empirical Model}

Discharge Probabilities: Our empirical discharge model builds on the theoretical model outlined in equation (1). Consistent with the linear probability model outlined above, we assume that exogenous discharge factors ( $\epsilon$ in equation $(1)$ ), which are not affected by financial incentives, are uniformly distributed, allowing us to express the probability of a discharge as follows:

$$
\operatorname{Pr}\left[D=1 \mid e^{S N F}, e^{r e s}\right]=D^{e x o g}+\alpha \times e^{S N F}\left(\operatorname{FinInc}^{S N F}(\tau, o c)\right)+\beta \times e^{\text {res }}\left(\operatorname{FinInc}^{\text {res }}(\tau)\right) .
$$

Here, $D$ denotes any discharge, which includes endogenous community discharges (our focus) but also discharges to a hospital, a different nursing home, or death, captured in an exogenous discharge rate $D^{e x o g}$. We extend our model of community discharges to overall discharges in order to capture the profit motives of nursing homes, which do not depend on the discharge reason, more accurately. While consumer payoffs depend on the discharge destination, we show below that the marginal benefit of resident effort remains unchanged in this extended framework. We define a period to be one week.

Resident's Effort Choice: In order to derive the resident's optimal effort choice, and to predict how discharge efforts and probabilities change in counterfactual policy experiments, we need to specify the resident's financial benefit from discharge. To this end, we assume 
the following indirect conditional utility:

$$
W(\tau, D, \eta)=\left\{\begin{array}{ll}
u-\kappa \times p^{\tau}+\eta^{S N F} & \text { if } D=0 \\
\eta^{\text {home }} & \text { if } D=1
\end{array},\right.
$$

where $u$ is the resident's gross utility from a week of nursing home care, and we normalize utility from home health care to zero. To simplify notation, we set the utility from discharge equal to the utility from home discharge, $\eta^{\text {home }}$. Utility differences between discharge reasons will not affect optimal effort choices as shown below.

We aim to match the discharge profiles for private payers and residents who transition to Medicaid and assume that they have the same relative utility for nursing home care, $u$. As outlined in Section 6.2, better access to community based care among private payers would imply $u^{P}<u^{M}$ and suggest that our baseline estimates might overstate the role of financial resident incentives. We return to this point in Section 8.3. $\kappa$ is a price coefficient, which maps the per-period price paid by the resident, $p^{\tau}$, into utility. $\eta^{S N F}$ and $\eta^{\text {home }}$ are type I extreme value taste shocks that are observed by the resident before choosing the effort level and unobserved by the SNF.

Residents choose the optimal discharge effort given by:

$$
\begin{aligned}
e^{r e s, *}=\arg \max _{e^{r e s} \geq 0}\left\{\operatorname{Pr}\left[D=1 \mid \cdot, e^{r e s}\right] \times W(\tau, D=1, \eta)\right. & +\left(1-\operatorname{Pr}\left[D=1 \mid \cdot, e^{r e s}\right]\right) \\
& \left.\times W(\tau, D=0, \eta)-\kappa \times c\left(e^{r e s}\right)\right\}
\end{aligned}
$$

where $c(e)$ denotes the cost of effort, measured in dollars. To translate the cost of effort back into utility, we multiply the cost by the price coefficient $\kappa$. The discharge probability is conditional on $D^{\text {exog }}$ and the resident's beliefs about $e^{S N F}$, captured by "." in $\operatorname{Pr}[D=$ $\left.1 \mid \cdot, e^{r e s}\right]$. However, these factors do not affect the resident's optimal effort because of the uniform distribution of $\epsilon$, see equation (1), shutting down potential free-riding incentives, as shown by the first order condition:

$$
e^{r e s, *}(\tau, \eta)=\left\{\begin{array}{lc}
c_{e}^{-1}\left(\frac{\beta}{\kappa} \times(W(\tau, D=1, \eta)-W(\tau, D=0, \eta))\right) & \text { if } W(\tau, D=1, \eta) \\
0 & >W(\tau, D=0, \eta) \\
\text { otherwise }
\end{array}\right.
$$

where $c_{e}^{-1}(\cdot)$ is the inverse marginal cost of effort function. 
Provider's Effort Choice: Turning to the LTC provider, we assume that the SNF does not observe the resident's taste shocks nor the resident's discharge effort. Instead, the nursing home observes the payer type of the focal resident and forms expectations over her optimal effort level. Therefore, we assume that nursing homes maximize over effort under the following belief:

$$
\operatorname{Pr}\left[D=1 \mid e^{S N F}, \tau\right]=D^{\text {exog }}+\alpha \times e^{S N F}+\beta \times E_{\eta}\left[e^{r e s, *} \mid \tau\right]
$$

To derive the optimal provider effort, $e^{S N F, *}$, we impose the following timing of events. During the period (week), providers choose their discharge effort level $e^{S N F}$ and realize the weekly flow payoff:

$$
\pi(\tau)=\left\{\begin{array}{ll}
-c\left(e^{S N F}\right) & \text { if bed is empty: } \tau=0 \\
r^{\tau}-m c-c\left(e^{S N F}\right) & \text { otherwise }
\end{array},\right.
$$

where $r^{\tau}$ is the private fee or Medicaid reimbursement rate, $m c$ is the weekly marginal cost of providing LTC, and $c\left(e^{S N F}\right)$ is the cost of effort measured in dollars. Discharges, arrivals, and payer type transitions are random events which realize at the end of the period. Arrivals and payer type transitions are exogenous and characterized by the weekly refill probability $\Phi$, see equation (4) and the transition probability $\psi$, respectively. Discharges, in contrast, depend on endogenous discharge efforts as outlined in equation (9) and together with arrivals determine the occupancy rate in other beds oc.

To simplify the analysis, we assume that discharge managers do not coordinate their discharge efforts between residents (beds). In other words, discharge managers do not internalize the effect of their "focal" discharge decision on the occupancy rate and discharges in other beds, which are both endogenous equilibrium objects. Instead, we assume that, in equilibrium, the discharge manager takes the time series process of the occupancy rate in other beds as given and chooses the discharge effort in the focal bed optimally. To reduce the state space, we model occupancy rate transitions as a Markov process, which is characterized by a week-to-week transition matrix, $\Theta$. This transition matrix denotes the conditional probability mass function over next week's occupancy rate in other beds, $o c^{\prime}$, conditional on today's occupancy rate on other beds, oc: $\Theta\left(o c, o c^{\prime}\right)=\operatorname{Pr}\left[o c^{\prime} \mid o c\right] .{ }^{28}$

Combining the timing assumptions with the assumptions on arrivals, discharges, and

\footnotetext{
${ }^{28}$ Conceptually, $o c$ refers to the occupancy in other beds. In practice, we approximate $o c$ by the overall occupancy rate in the structural estimation. We also assume that discharge managers form beliefs over next week's occupancy rate based on the current occupancy rate only. That simplification implies that the discharge manager does not condition on the payer type distribution nor the payer type in the focal bed.
} 
payer type transitions, we can express the SNF's optimal discharge efforts through the following Bellman equation:

$$
V(\tau, o c)=\max _{e^{S N F} \geq 0}\left\{\pi(\tau)-c\left(e^{S N F}\right)+\delta E\left[V\left(\tau^{\prime}, o c^{\prime}\right) \mid \tau, o c, e^{S N F}\right]\right\}
$$

where $\delta$ is a discount factor and

$$
\begin{aligned}
E\left[V \mid 0, o c, e^{S N F}\right]= & \sum_{o c^{\prime}} \Theta\left(o c, o c^{\prime}\right) \times\left[\left(1-\Phi\left(o c^{\prime}\right)\right) \times V\left(0, o c^{\prime}\right)\right. \\
& \left.+\Phi\left(o c^{\prime}\right) \times\left(\rho V\left(P, o c^{\prime}\right)+(1-\rho) V\left(M, o c^{\prime}\right)\right)\right] \\
E\left[V \mid M, o c, e^{S N F}\right]= & \sum_{o c^{\prime}} \Theta\left(o c, o c^{\prime}\right) \times\left[\left(1-\operatorname{Pr}\left[D=1 \mid e^{S N F}, M\right]\right) \times V\left(M, o c^{\prime}\right)\right. \\
& +\operatorname{Pr}\left[D=1 \mid e^{S N F}, M\right] \times\left(\left(1-\Phi\left(o c^{\prime}\right)\right) \times V\left(0, o c^{\prime}\right)\right. \\
& \left.\left.+\Phi\left(o c^{\prime}\right) \times\left(\rho V\left(P, o c^{\prime}\right)+(1-\rho) V\left(M, o c^{\prime}\right)\right)\right)\right] \\
E\left[V \mid P, o c, e^{S N F}\right]= & \sum_{o c^{\prime}} \Theta\left(o c, o c^{\prime}\right) \times\left[\left(1-\operatorname{Pr}\left[D=1 \mid e^{S N F}, P\right]\right) \times\left((1-\psi) V\left(P, o c^{\prime}\right)\right.\right. \\
& \left.+\psi V\left(M, o c^{\prime}\right)\right)+\operatorname{Pr}\left[D=1 \mid e^{S N F}, P\right] \times\left(\left(1-\Phi\left(o c^{\prime}\right)\right) \times V\left(0, o c^{\prime}\right)\right. \\
& \left.\left.+\Phi\left(o c^{\prime}\right) \times\left(\rho V\left(P, o c^{\prime}\right)+(1-\rho) V\left(M, o c^{\prime}\right)\right)\right)\right] .
\end{aligned}
$$

In words, the value function combines the flow profit net of the cost of effort and a continuation value, see equation (10). The continuation value of an empty bed, as indicated in equation (11), is given by the probability of drawing a new resident, captured by the refill probability vector $\Phi\left(o c^{\prime}\right)$, multiplied by the payer type probability at admission. For example, the new resident is a private payer with probability $\rho$ delivering a payoff vector of $V\left(P, o c^{\prime}\right)$. Furthermore, expectations are taken over next week's occupancy rate as indicated by the transition matrix $\Theta\left(o c, o c^{\prime}\right)$. The continuation value of a bed filled with a Medicaid beneficiary, see equation (12), adds to this the possibility that the focal resident may be

discharged, which depends on the efforts of the nursing home and the resident. Finally, the continuation value of a bed filled with a private payer, see equation (13), adds to this a payer type transition to Medicaid, which happens with probability $\psi$.

\subsection{Identification and Estimation}

Parameters estimated outside of the model: We estimate several objects of the empirical analysis outside of the model, which are summarized in panel A of Table 3. We estimate the weekly refill probabilities according to equation (4), see Figure D.6 in Appendix Section D.9. The week-to-week occupancy transition matrix is an endogenous equilibrium object. 
For the purpose of estimation, we use the empirical transition matrix. An excerpt is provided in Table C.1 in Appendix Section C. In the counterfactual exercises, we endogenize the transition matrix to allow for changes in discharge efforts affecting occupancy transitions, which in turn feed back into optimal effort choices.

We also estimate the probability of a payer type transition from private to Medicaid from observed week-to-week changes, which happens in $1.1 \%$ of cases. Excluding Medicare beneficiaries, about $78.2 \%$ of new residents initially pay out-of-pocket. To construct $D^{\text {exog }}$, we sum the average discharge rate to other destinations, e.g., hospitals, nursing homes, or mortality, see Figure D.1 in Appendix Section D.2. We simply take the unweighted average over occupancy and payer type specific discharge rates and find an average of $2.3 \%$. This calculation abstracts away from observed differences between payer types, which we attribute to ex post advantageous selection among Medicaid beneficiaries, see again Section 6.4 for a discussion. ${ }^{29}$ Finally, the private rate and the Medicaid reimbursement rate correspond to the average rates in Pennsylvania and California in the sample period.

Calibrated parameters: We set the weekly discount factor to $\delta=0.95^{1 / 52}$, as indicated in panel B of Table 3. We require a scale normalization on either the cost of effort or the return on effort since, naturally, they cannot be separately identified from our data. In the baseline analysis, we assume $c(e)=e^{2}$ and thereby load differences in cost functions between payer types onto differences in the returns of effort. ${ }^{30}$ In an alternative attempt, we define effort as a change in the probability of discharge by normalizing $\alpha=\beta=1$. Here, we allow for linear quadratic cost functions of effort, $c(e)=\gamma_{1} e+\gamma_{2} e^{2}$ and estimate separate cost parameters for patients and providers. This approach yields qualitatively similar results, see Appendix Section F.2 for details. Finally, we normalize the utility from nursing home care $u$ as we can only identify utility up to scale. This is because utility affects discharges through effort in our specification, which is again scaled by the factor $\beta$.

Parameters estimated within the model: Finally, we turn to key structural parameters for our analysis that we estimate using the model. These include the marginal cost of nursing home care per resident and day $m c$, the price coefficient $\kappa$ and the effort parameters $\alpha$ and $\beta$. Identification comes from the estimated discharge profiles in Figure 3. Specifically, $m c$ is identified at the occupancy threshold $o c^{*}$, i.e. the occupancy rate where the Medicaid discharge rate starts to increase, see Figure 1. At $o c^{*}$, the marginal benefit of effort to

\footnotetext{
${ }^{29}$ We abstract away from this as we do not model differences in health in the structural analysis. Importantly, these differences are considerably smaller than the differences in home discharges, which are captured in the model.

${ }^{30}$ To see this, consider the resident's optimal effort choice in equation (8). We observe the private rate $p^{P}$ as well as the discharge rates. Suppose we generalize the cost $c(e)=\gamma e^{2}$. Then we have $\frac{\partial \operatorname{Pr}[D=1 \mid \cdot]}{\partial p^{P}}=$ $\beta \times \frac{\partial e^{r e s, *}}{\partial p^{P}}=\frac{\beta^{2 *}}{2 \gamma}$. Hence, scaling up the cost parameter $\gamma$ would simply scale up $\beta$.
} 
discharge a Medicaid beneficiary equals the marginal cost of effort when evaluated at $e^{S N F}=$ $0=m c_{e}(0)$. Hence, the marginal benefit must be zero as well, which trades off the Medicaid flow profit $\pi(M)$ against the option value of drawing a new resident tomorrow. The option value increases in the refill probability. Intuitively, we can pin down the marginal cost that equates $\pi(M)$ with the option value when evaluated at $o c^{*}$.

The remaining parameters are identified from the observed home discharge rates for Medicaid beneficiaries and private payers at low and high occupancy rates. Intuitively, discharge rates at low occupancy rates, $o c<o c^{*}$, identify the resident coefficients, $\beta$ and $\kappa$, and the increase in discharge rates for Medicaid beneficiaries at higher occupancy rates, $o c \geq o c^{*}$, identifies the provider coefficient $\alpha$.

We estimate the parameters $\theta=(\alpha, \beta, \kappa, m c)$ by minimizing the sum of squared differences between discharge rates predicted by the model and observed home discharge rates:

$$
\hat{\theta}=\arg \min _{\theta} \sum_{\tau=P, M} \sum_{o c=65}^{99}\left(D_{\tau, o c}(\theta)-\hat{D}_{\tau, o c}\right)^{2}
$$

where $\hat{D}_{\tau, o c}$ denotes the estimated home discharge rates by payer type and occupancy shown in Figure $3 .^{31}$ We omit discharge rates at $o c=100$ to avoid potential measurement error. The estimation algorithm proceeds as follows: First, for an initial parameter guess $\theta_{0}$, we solve the provider value function given by equations (10) to (13) and the implied optimal effort function of the SNF via contraction mapping. This allows us to predict discharge rates $D_{\tau, o c}\left(\theta_{1}\right)$ using equation (9). We then update the parameter vector and iterate until the least squares criterion in equation (14) attains its minimum.

Inference: We conduct inference via bootstrap. One computational limitation of this procedure is that estimating the regression model (2) is very time and memory consuming due to the large number of fixed effects and about 15 million observations. This makes it challenging to estimate the model many times as required in a standard bootstrap procedure. Instead, we leverage the observation that the OLS estimator for the vector $\nu=\left[\gamma^{75}, \ldots, \gamma^{100}, \delta^{75}, \ldots, \delta^{100}\right]$ is jointly normally distributed, $\hat{\nu} \sim N(\nu, \Sigma)$, because of the central limit theorem. Therefore, we only estimate the variance-covariance matrix for entire vector, $\Sigma$, once and then draw discharge coefficients. For each bootstrap iteration $b=1, \ldots, B$, we draw $\hat{\nu}^{b} \sim N(\hat{\nu}, \hat{\Sigma})$ and then re-estimate the parameters $m c, \alpha, \beta$ and $\kappa$. Finally, we obtain $95 \%$ confidence intervals by ordering bootstrapped parameters, which are re-centered around the respective point estimates, and reporting the 2.5 th and the 97.5 th percentile. We set $B=99$.

\footnotetext{
${ }^{31}$ Hansen (2017) uses a similar objective function.
} 


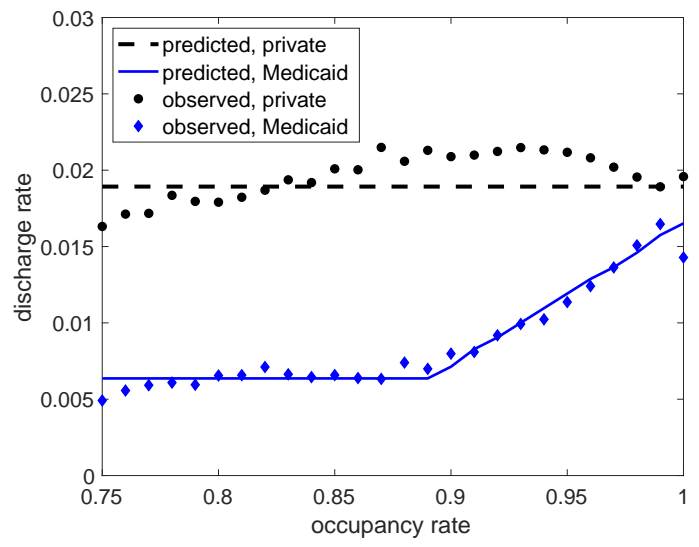

(a) Actual Polices

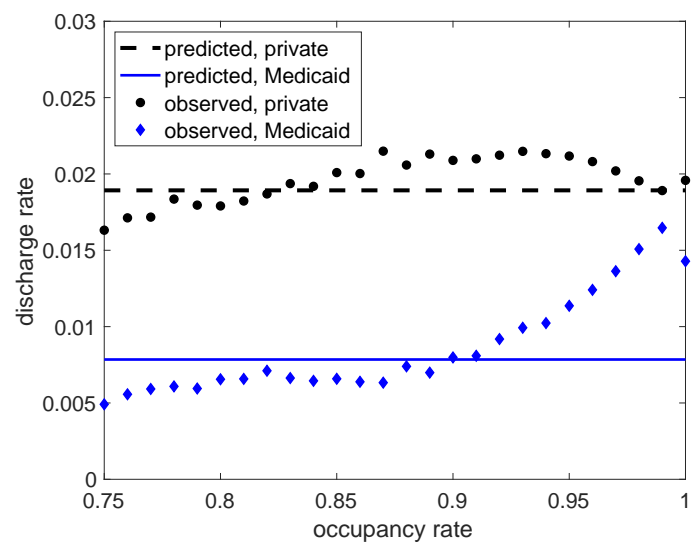

(c) Top-Up Payment

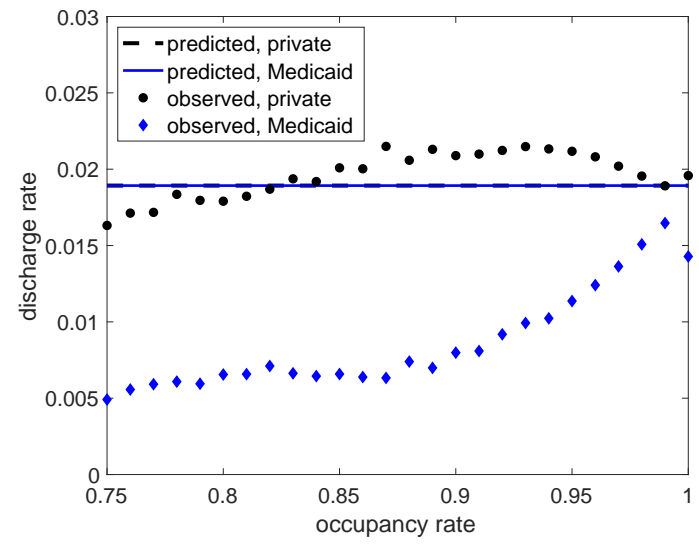

(b) Medicaid Voucher

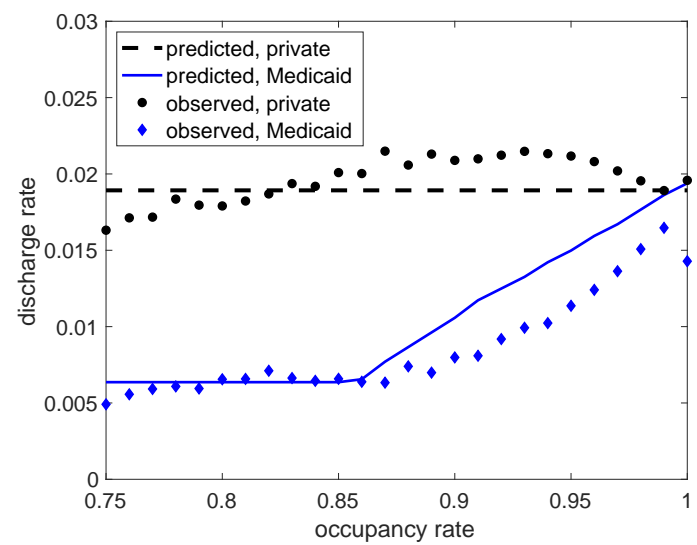

(d) Front-Loaded Medicaid Reimbursement

Figure 6: Simulated Discharge Rates Under Different Policies

\section{Model Results and Policy Counterfactuals}

In this section, we first discuss the model fit and model parameter estimates, and then use these results to conduct policy counterfactual simulations.

\subsection{Estimates of Model Parameters and Model Fit}

The model provides a very good fit to the observed community discharge rates, as shown in Figure 6a. The circles and diamonds denote the estimated discharge rates from Figure 3, and the lines denote the analogous model fit.

We estimate the marginal costs per resident and day at $\$ 77$. This estimate is smaller than marginal cost estimates from the literature. For example, Hackmann (2017) estimates an average marginal cost of $\$ 159$ using data from Pennsylvania over the entire resident population. Since we focus on a healthier marginal population of nursing home residents that are discharged back to the community, our smaller marginal cost estimate is plausible. 
Table 3: Structural Parameter Estimates

\begin{tabular}{|c|c|c|c|c|}
\hline & \multirow{2}{*}{$\begin{array}{c}\text { Full Sample } \\
\text { (1) }\end{array}$} & \multirow{2}{*}{$\begin{array}{c}\text { CA\&PA Sample } \\
(2)\end{array}$} & \multicolumn{2}{|c|}{ By SNF Incentives } \\
\hline & & & $\begin{array}{c}\text { High } \\
(3)\end{array}$ & $\begin{array}{l}\text { Low } \\
(4)\end{array}$ \\
\hline \multicolumn{5}{|l|}{ A. Estimated Outside of Model } \\
\hline Refill Probability $\Phi(o c)$ & \multicolumn{4}{|c|}{ See Figure D.6. } \\
\hline Occupancy Trans. Matrix $\Theta\left(o c, o c^{\prime}\right)$ & \multicolumn{4}{|c|}{ See Table C.1. } \\
\hline $\operatorname{Pr}[$ Transition to Medicaid] $\psi$ & $1.1 \%$ & $1.2 \%$ & $1.1 \%$ & $1.3 \%$ \\
\hline $\operatorname{Pr}[$ Private Payer at Admission] $\rho$ & $78.2 \%$ & $80.9 \%$ & $83.6 \%$ & $71.6 \%$ \\
\hline Exogenous Discharge Rate $D^{\text {exog }}$ & $2.3 \%$ & $2.8 \%$ & $2.7 \%$ & $2.8 \%$ \\
\hline Daily Private Rate $r^{P}$ & $\$ 194$ & $\$ 194$ & $\$ 213$ & $\$ 172$ \\
\hline Daily Medicaid Rate $r^{M}$ & $\$ 159$ & $\$ 159$ & $\$ 157$ & $\$ 160$ \\
\hline \multicolumn{5}{|l|}{ B. Calibrated } \\
\hline Discount Factor $\delta$ & \multicolumn{4}{|c|}{$0.95^{\frac{1}{52}}$} \\
\hline Cost of Effort Function $c(e)$ & \multicolumn{4}{|c|}{$e^{2}$} \\
\hline Utility of Nursing Home Care $u$ & \multicolumn{4}{|c|}{5} \\
\hline \multicolumn{5}{|l|}{ C. Estimated Inside of Model } \\
\hline \multirow[t]{2}{*}{ SNF Effort Parameter $\alpha$} & 0.0314 & 0.0375 & 0.0398 & 0.0672 \\
\hline & {$[0.0267,0.0326]$} & {$[0.0322,0.0395]$} & {$[0.0358,0.0463]$} & {$[0.0592,0.076]$} \\
\hline \multirow[t]{2}{*}{ Resident Effort Parameter $\beta$} & 0.372 & 0.397 & 0.387 & 0.37 \\
\hline & {$[0.34,0.504]$} & {$[0.32,0.435]$} & {$[0.297,0.458]$} & {$[0.275,0.432]$} \\
\hline \multirow[t]{2}{*}{ Resident Price Coefficient $\kappa$} & 0.116 & 0.0822 & 0.102 & 0.0519 \\
\hline & {$[0.00712,0.14]$} & {$[0.0624,0.102]$} & {$[0.0621,0.134]$} & {$[0.0161,0.0796]$} \\
\hline \multirow[t]{2}{*}{ Marginal Cost of Care $m c$} & 77 & 96 & 65 & 110 \\
\hline & {$[77,77.4]$} & {$[81,167]$} & {$[31,99]$} & {$[85,124]$} \\
\hline SNF Elasticity $\epsilon^{S N F}$ & 1.62 & 2.04 & 1.13 & 2.40 \\
\hline Resident Elasticity $\epsilon^{r e s}$ & 0.525 & 0.532 & 0.761 & 0.220 \\
\hline
\end{tabular}

Notes: The table is organized into three panels. The first panel summarizes the objects that are estimated outside of the model. The refill probability $\Phi\left(\right.$ oc) and the occupancy transition matrix $\Theta\left(o c, o c^{\prime}\right)$ are estimated separately for each sample in columns (1) to (4). The second panel presents parameters that are calibrated. The second panel presents parameters that are calibrated. The third panel presents the parameters that are estimated inside the model. Inference is conducted via a bootstrap procedure.

Both estimated discharge effort parameters are positive, $\hat{\alpha}>0$ and $\hat{\beta}>0$, implying that provider and resident discharge efforts increase the discharge probability. To assess the relative importance of provider and patient incentives, we simulate the effect of a $1 \%$ change in financial incentives on the length of stay holding discharge efforts of the opposite market side fixed. Starting with patients, we quantify the effect of a $1 \%$ increase in private rate on the discharge probability for private payers and then simulate the change in the expected length of stay holding the supply side fixed. Turning to nursing homes, we consider the effect of a $1 \%$ increase in the Medicaid reimbursement rate on provider discharge efforts and the length of stay of Medicaid beneficiaries, holding the demand side fixed.

Combining the relative changes in prices and utilization, we find a provider elasticity 
Table 4: Simulated Length of Stay and Cost Savings Under Policy Counterfactuals

\begin{tabular}{lccccc}
\hline & Actual & Voucher & Top-up & $1 \%$ Front & $10.5 \%$ Front \\
\hline Private LOS & 23.76 & 23.76 & 23.76 & 23.76 & 23.76 \\
Medicaid LOS & 31.32 & 23.64 & 35.13 & 30.28 & 23.81 \\
Average Occupancy & $91.1 \%$ & $86.9 \%$ & $93.1 \%$ & $90.5 \%$ & $87.5 \%$ \\
$\Delta$ Medicaid LOS & & -7.68 & 3.81 & -1.04 & -7.51 \\
$\Delta$ Spending per Stay in $\$$ & & 296 & 11,354 & -153 & -1104 \\
$\Delta$ Annual Spending in bn\$ & & 0.047 & 1.836 & -0.025 & -0.180 \\
\hline
\end{tabular}

Notes: The table summarizes the length of stay (LOS) in weeks, average occupancy rates, Medicaid savings per stay, and national Medicaid savings for the three counterfactual policy experiments.

of 1.6 and a patient elasticity of 0.5 . Consistent with the reduced form evidence, we find that providers respond about three times as elastically to incentives than patients. Both elasticities exceed the reduced form analogues largely because we consider a marginal change in prices in this simulation exercise. Repeating the analysis with a $100 \%$ price change for patients and a $24 \%$ change for providers, the patient elasticity falls to 0.37 and the provider elasticity falls to $1.1 .^{32}$

\subsection{Cost Savings and Policy Counterfactuals}

\subsubsection{Potential Cost Savings}

Before turning to the policy counterfactuals, we consider the scope for cost savings in this context. To this end, we start by comparing Medicaid spending on nursing home care to expenditures that would arise when discharged nursing home residents lived in the community instead. These expenditures consist of home health care and spending on other medical care but also the cost of living expenditures including housing, food, etc. We note that Medicaid will cover only a fraction of these community expenditures. Therefore, our cost savings can be interpreted as overall LTC savings and as a lower bound on Medicaid savings. Using data from the Medical Expenditure Panel Survey and the Consumer Expenditure Survey, we find mean annual expenditures of $\$ 1,018$ for formal home health care and of $\$ 12,903$ for all other expenditures in the age group 80 and over.

We also add the opportunity cost of informal care provided by family members. Using

\footnotetext{
${ }^{32}$ Mechanically, the "marginal" elasticities exceed the "non-marginal" elasticities for two reasons. First, the curvature in the effort function implies that larger changes in incentives lead to relatively smaller adjustments in effort. Second, the curvature in the patient's utility taste shocks implies that larger reductions in private rates move the taste shock threshold for a discharge preference further in the tail of distribution with a declining density (fewer marginal cases).
} 
data from the HRS, we find that the average individual receives 39 hours of informal care per month while the average conditional on receiving any informal care equals 92 hours. These values are roughly similar to previous findings in the literature. To calculate the opportunity cost of informal care, we use median weekly earnings from the Bureau of Labor Statistics for men and women for the years 2000 to 2005. Using the fact that $71 \%$ of informal caregivers of HRS respondents are women and assuming a 40-hour work week, we calculate an average hourly wage of $\$ 13.72$. This implies annual opportunity costs of informal care giving of $\$ 14 \times 39 \times 12=\$ 6,552$. Taken together, the expenditures and opportunity costs of home health care equal $\$ 1,018+\$ 12,903+\$ 6,552=\$ 20,473$ per year or $\$ 56$ per person and day. We note that our estimates on formal and informal community based care may overstate the expenditures for our sample popualation since many marginal nursing home residents do not receive home health care (see the results in Figure 5.)

Nevertheless, this estimate is considerably smaller than the average daily Medicaid reimbursement rate of $\$ 159$ and smaller than the estimated marginal cost of nursing home care of $\$ 77$. Medicaid spending could therefore be lowered by at least $(\$ 159-\$ 56) \times 7$ days $=\$ 721$ per resident if the beneficiary was discharged one week earlier. The first column of Table 4 indicates that Medicaid stays exceed the length of stay of private payers by 7.6 weeks on average. Since we find no evidence that longer stays lead to health improvements, this difference points to Medicaid overspending of $7.6 \times \$ 721=\$ 5,480$ per Medicaid stay or about $18.6 \%$ of Medicaid spending per nursing home stay. ${ }^{33}$ Multiplying this fraction with national Medicaid nursing home spending of $\$ 55 \mathrm{bn}$ in 2015 suggests annual savings of up to $\$ 10.2 \mathrm{bn}$.

To estimate the national savings more conservatively, we calculate the overall savings in our sample population and scale the estimates by a factor of 5 , since the four states in our sample account for about $20 \%$ of overall Medicaid nursing home spending. ${ }^{34}$ Combining a $18.6 \%$ reduction in Medicaid spending with a base of $1 \mathrm{~m}$ Medicaid weeks per year in our sample, we calculate annual savings of $\$ 0.2 \mathrm{bn}$ and $\$ 1.0 \mathrm{bn}$ in our sample states and at the national level, respectively.

\subsubsection{Policy Counterfactuals}

We consider three policy counterfactuals that change the discharge incentives for SNFs and nursing home residents. When simulating their effects on the length of stay and Medicaid spending, we take endogenous changes in occupancy rates into account, which in turn affect provider discharge efforts. To this end, we divide the nursing home into two wings. The additional wing allows us to incorporate admissions and discharges among residents that were excluded from the estimation sample but also affect overall occupancy. We treat these

\footnotetext{
${ }^{33}$ Overall Medicaid spending equals 31.3 weeks $\times 7$ days $\times \$ 159=\$ 34,859$.

${ }^{34}$ See http://files.kff.org/attachment/Infographic-Medicaids-Role-in-Nursing-Home-Care.
} 
admissions and discharges as exogenous. For the study population (nursing home wing) of interest, we take observed weekly admissions as exogenous, and use our structural model to predict discharge rates under alternative policy regimes. Combining admission and discharge profiles between wings allows us incorporate the effect of policy changes on occupancy rates. In the simulation analysis, we then add an outer loop to the optimization problem, which searches for a fixed point in the discharge profiles, see Appendix Section E for details.

Voucher Program: First, we consider the introduction of a voucher program, which equates the marginal prices for nursing home care between Medicaid beneficiaries and private payers, $p^{M}=p^{P}$. We compensate Medicaid beneficiaries for their expected outlays through a lump-sum transfer, which equals the average length of stay of private payers, 23.6 weeks, times the weekly private rate. This amounts to a lump-sum of $\$ 32,144$ per Medicaid stay. The program affects resident and provider incentives in opposing directions. On the one hand, Medicaid beneficiaries now have a larger incentive to end their stays early. On the other hand, SNFs are now indifferent between private and Medicaid residents because they generate identical profits. Therefore, nursing homes will reduce their discharge effort for Medicaid beneficiaries to zero.

Figure 6b shows that Medicaid beneficiaries and private payers are now discharged at the same rate (the discharge rates lie on top of each other). As indicated in the second column in Table 4, the length of stay for Medicaid beneficiaries is reduced to 23.6 weeks, which also reduces the average occupancy rate to $86.9 \%$. Medicaid saves 31.3 weeks of nursing home payments worth $\$ 34,859$ but provides transfers worth $\$ 32,144$ under this policy. We add costs for the additional 7.7 weeks spent in the community, worth 7.7 weeks $\times 7$ days $\times \$ 56=\$ 3,011$ per Medicaid stay. Hence, the overall increase in expenditures equals $\$ 32,144+\$ 3,011-$ $\$ 34,859=\$ 296$ per Medicaid stay, corresponding to a $0.85 \%$ increase in Medicaid spending. Using the conservative approach we find an increase in annual Medicaid expenditures of $0.85 \% \times 1 \mathrm{~m}$ weeks $\times 7$ days $\times \$ 159 \times 5=\$ 0.047 \mathrm{bn}$.

Top-Up Program: Next, we consider a top-up policy, where Medicaid residents pay the difference between the private fee and the Medicaid reimbursement rate, $p^{P}-p^{M}$, outof-pocket. This equates the revenues among payer types and affects provider and patient incentives in opposing directions, as discussed in the voucher counterfactual.

Figure 6c shows that home discharge rates among Medicaid residents at low occupancies are slightly higher than under current policies (compared to Figure 6a) due to residents' increased discharge incentives. In contrast to current policies, we do not observe an increase in Medicaid discharges at high occupancies under top-up payments since both payer types are equally profitable for nursing homes. The incentive effects for providers outweigh the 
incentive effects for patients, leading to an overall increase in the length of stay by 3.8 weeks and a 2 percentage point rise in the average occupancy rate, see the third column in Table 4. The effect on spending is twofold. First, Medicaid has to cover 3.8 extra weeks of nursing home care leading to additional expenses of $(\$ 159-\$ 56) \times 7$ days $\times 3.8$ weeks $=\$ 2,747$. On top of that, Medicaid may have to reimburse the patient expenditures stemming from the difference between the private and the Medicaid rate. This adds another $(\$ 194-\$ 159) \times$ 7 days $\times 35.1$ weeks $=\$ 8,607$. Taken together, this policy may increase spending by $\$ 11,354$ per Medicaid enrollee, or $\$ 1.84$ bn overall.

Episode-Based Reimbursements: Finally, we simulate the effects of a small shift from per-diem reimbursement to an episode-based reimbursement approach. In this counterfactual, we reduce the daily Medicaid reimbursement rate by $1 \%$ and compensate the provider for the forgone variable profits with an up-front payment. This compensation maintains the profitability of Medicaid beneficiaries and counteracts a provider's incentive to respond along other margins such as changing the quality of care, see Hackmann (2017). Specifically, the change in variable profits combines two components, described below:

$$
\begin{aligned}
& \Delta_{1}=(\$ 159-\$ 56) \times 7 \times \Delta \text { weeks } \\
& \Delta_{2}=1 \% \times \text { weeks }^{\text {new }} \times \$ 159
\end{aligned}
$$

The first component, $\Delta_{1}$, denotes the Medicaid rate markup over marginal costs times the change in the length of stay. The second component captures the lower Medicaid rates for the counterfactual length of stay. Finally, the overall compensation, $\Delta^{1 \%}$, is simply the sum of the two components. We then replace $V\left(M, o c^{\prime}\right)$ by $V\left(M, o c^{\prime}\right)+\Delta^{1 \%}$ in equations (11) and (13). Intuitively, the provider receives the up-front compensation whenever a new Medicaid beneficiary arrives or a private payer transitions into Medicaid.

The simulated discharge rates in Figure 6d suggest a large increase in provider discharge efforts. The new kink point is at $86 \%$ occupancy instead of $89 \%$ under the status quo. We find a reduction in length of stay of Medicaid beneficiaries of about 1.04 weeks and a corresponding decline in average occupancy to $90.5 \%$, as indicated in the last column of Table 4. Taking the compensation payments into account, changes in Medicaid spending per nursing home stay are given by the difference between the marginal cost of nursing home care and the cost of alternative community care scaled by the 1.04 week change in the length of stay. This suggest savings of about $(\$ 77-\$ 56) \times 7$ days $\times 1.04$ weeks $=\$ 153$ under this policy, and total annual spending is reduced by at least $\$ 0.025$ billion. ${ }^{35}$ This policy, which

\footnotetext{
${ }^{35}$ More precisely, Medicaid savings equal $\$ 159-\$ 56$ per reduced day of nursing home care plus the $1 \%$ reimbursement savings on remaining weeks of nursing home care, $\Delta_{2}$. Subtracting the compensation
} 
increases financial discharge incentives among providers, therefore achieves the largest cost saving among the counterfactuals considered here.

Furthermore, we find that transitioning $10.5 \%$ of per-diem payments to an up front episode-based reimbursement is as effective as the voucher program in reducing the average length of a Medicaid stay to the average length of a private stay, see the last column in Table 4. However, the implied cost savings are substantially larger. In this case we find cost savings worth $\$ 1,104$ per stay or about $\$ 0.18$ billion in total annual savings. Therefore, we conclude that transitioning to an episode-based reimbursement model is more effective than increasing resident cost-sharing in shortening the length of Medicaid stays. Note that none of the policy counterfactual considered can reach the full cost savings potential of $\$ 1.0 \mathrm{bn}$ because we compensate patients and providers for increased prices and lower reimbursement rates.

\subsection{Discussion}

Our structural analysis places strong assumptions on the data generating process, which affect the quantitative findings in counterfactual exercises. In this section, we revisit the robustness of our main findings with respect to the main identification concerns raised in Sections 6.2 and 6.3 .

Unobserved patient heterogeneity: With respect to unobserved patient heterogeneity, we note that most concerns point to better access to community care for private payers. In this case, our baseline findings overstate the role of patient incentives in the counterfactual analysis. We revisit the role of patient incentives in additional robustness exercises that build on estimated elasticities from the literature. Following, Manning et al. (1987), Finkelstein et al. (2012), and Shigeoka (2014), we assume a patient elasticity of 0.2 following a 100\% reduction in private rates. Specifically, we allow for differences in patient utility over nursing home care $u^{P}<u^{M}$ in equation (6), which can at least partially reconcile observed differences in discharge profiles at low occupancies. We continue to normalize $u^{M}=5$ as outlined above and estimate $u^{P}$ by adding an outer loop to the estimation routine that approximates the implied patient elasticity to 0.2 .

We find that $u^{P}$ falls short of $u^{M}$ by $30 \%$, see Table F.1 in Appendix Section F.1 for the estimated parameters and elasticities. To reconcile the unchanged differences in discharge rates, we also find that the price coefficient scaled by the effort coefficient, $\beta \times \kappa$ drops by $55 \%$. This suggests that $25 \%$ of the observed differences in discharge rates can now be attributed to unobserved patient heterogeneity. As expected, these findings point to

payments to nursing homes $\Delta^{1 \%}=\Delta_{1}+\Delta_{2}$ yields net savings of $\$ 77-\$ 56$ per reduced day of nursing home care. 
an even more important role of provider incentives when compared to patient incentives. The provider elasticity now exceeds the patient elasticity by a factor of 6 and we find that a voucher program leads to an increase in the length of Medicaid stays whereas shifting provider reimbursement towards an episode-based payment system is more than twice as effective in shortening Medicaid stays than under the baseline, see Table F.2 in Appendix Section F.1.

Provider heterogeneity: Our structural model abstracts away from provider heterogeneity and considers the trade-offs for a representative nursing home instead. To this end, we purge the data off provider (and patient) heterogeneity by controlling for nursing home year fixed effects, as well as week of stay and other patient characteristics, and fit the model to the residualized data. Following a Jensen's inequality argument, a potential concern is that heterogeneity in provider responses to occupancy variation may bias the "mean" estimates for the representative firm, when fitting the nonlinear provider problem to the "average" discharge profiles. To investigate this possibility, we use the price data from California and Pennsylvania and split the sample into nursing homes with higher and lower than median private rate markups over Medicaid rates, as described in Section 6.3. We also revisit the primitives estimated outside the model for each subsample and estimate the structural model for each subsample.

The structural parameter estimates are presented in columns (3) and (4) of Table 3. We benchmark these estimates to the estimates presented in column (2), which presents the structural parameters for the entire sample of nursing homes in Pennsylvania and California only. Averaging the estimates from columns (3) and (4), we find very similar parameter estimates to those in column (2) deviating by at most $10 \%$. One exception is the SNF effort parameter from column (2), which falls short of the averages between samples by $42 \%$. This difference is partially offset by differences in marginal costs. Most importantly, we find very similar patient and provider elasticities in this comparison.

Finally, we also revisit the robustness exercises discussed in the preliminary analysis using the structural model, see Appendix Section F.1 for details.

\section{Conclusion}

How do patient and provider incentives affect long term care utilization and health care spending? We answer this question in the context of the U.S. nursing home industry. Our findings indicate that resident cost-sharing incentives and profit incentives of nursing homes affect the length of nursing home stays among relatively healthy residents who can return to the community.

We find no evidence for improved health outcomes from longer stays. If anything our 
findings point in the opposite direction, suggesting that a substantial part of the Medicaid expenditures that burden state budgets may be wasteful. Our evidence on provider incentives suggests that excess capacity of nursing homes may contribute towards excessive nursing home utilization, providing an argument for Certificate of Need laws, which restrict entry and capacity investments of nursing homes. This observation is consistent with an older claim made by Roemer (1961) on provider financial incentives in hospitals: "in an insured population, a hospital bed built is a filled bed."

Many states have recognized that it is more cost-effective to reimburse home health care for Medicaid-eligible seniors instead of paying for long nursing home stays. Programs that are authorized under section 1915(c) waivers provide funding for community-based LTC. Most of these programs were started after our sample period that ends in 2005, but our results can nevertheless speak to the likely success of these new policies. While these programs largely affect patient incentives, our findings indicate that incentivizing providers may be more cost-effective in reducing nursing home utilization. Specifically, our findings suggest that transitioning from per-diem to episode-based provider reimbursement is more effective than increasing resident cost-sharing in shortening Medicaid stays. To investigate the effects of recent policy changes, we intend to expand our analysis using more recent data in future research and to account for different implementations of LTC vouchers.

\section{References}

Arling, Greg, Kathleen A. Abrahamson, Valerie Cooke, Robert L. Kane, and Teresa Lewis. 2011. "Facility and Market Factors Affecting Transitions From Nursing Home to Community." Medical Care 49 (9):790-796.

Aron-Dine, Aviva, Liran Einav, and Amy Finkelstein. 2013. "The RAND Health Insurance Experiment, Three Decades Later." Journal of Economic Perspectives 27 (1):197-222.

Borella, Margherita, Mariacristina De Nardi, and Eric French. 2017. "Who Receives Medicaid in Old Age? Rules and Reality." Unpublished manuscript.

Busso, Matias, John DiNardo, and Justin McCrary. 2014. "New Evidence on the Finite Sample Properties of Propensity Score Reweighting and Matching Estimators." Review of Economics and Statistics 96 (5):885-897.

Cai, Shubing, Dana B Mukamel, Peter Veazie, and Helena Temkin-Greener. 2011. "Validation of the Minimum Data Set in identifying hospitalization events and payment source." Journal of the American Medical Directors Association 12 (1):38-43.

Chapin, Rosemary, Deborah Schild Wilkinson, Roxanne Rachlin, Michelle Levy, and Rachel Lindbloom. 1998. "Going Home: Community Reentry of Light Care Nursing Facility Residents Age 65 and Over." Journal of Health Care for the Poor and Underserved 25 (2):35- 
48.

Ching, Andrew T, Fumiko Hayashi, and Hui Wang. 2015. "Quantifying the impacts of limited supply: The case of nursing homes." International Economic Review 56 (4):1291-1322.

Clemens, Jeffrey and Joshua D Gottlieb. 2014. "Do physicians' financial incentives affect medical treatment and patient health?" American Economic Review 104 (4):1320-49.

Congressional Budget Office. 2013. "Rising demand for long-term services and supports for elderly people." .

Cutler, David M. 1995. "The incidence of adverse medical outcomes under prospective payments." Econometrica 63:29-50.

Cutler, David M and Richard J Zeckhauser. 2000. "The anatomy of health insurance." 1:563-643.

Dickstein, Michael. 2015. "Physician vs. Patient Incentives in Prescription Drug Choice." Unpublished manuscript.

Doyle, Joseph J., John A. Graves, and Jonathan Gruber. 2017. "Uncovering Waste in US Healthcare." Journal of Health Economics 54:25-39.

Dummit, Laura, Grecia Marrufo, Jaclyn Marshall, Ellen Tan, Aylin Bradley, Cornelia Hall, Younyoung Lee et al. 2018. "CMS bundled payments for Care Improvement Initiative models 2-4: Year 5 evaluation \& monitoring annual report." https://downloads.cms.gov/files/cmmi/bpci-models2-4-yr5evalrpt.pdf .

Einav, Liran, Amy Finkelstein, and Neale Mahoney. forthcoming. "Provider Incentives and Healthcare Costs: Evidence from Long-Term Care Hospitals." Econometrica .

Eliason, Paul J., Paul L. E. Grieco, Ryan C. McDevitt, and James W. Roberts. forthcoming. "Strategic Patient Discharge: The Case of Long-Term Care Hospitals." American Economic Review .

Ellis, Randall P and Thomas G McGuire. 1993. "Supply-side and demand-side cost sharing in health care." Journal of Economic Perspectives 7 (4):135-151.

Finkelstein, Amy, Sarah Taubman, Bill Wright, Mira Bernstein, Jonathan Gruber, Joseph P Newhouse, Heidi Allen, Katherine Baicker, and Oregon Health Study Group. 2012. "The Oregon health insurance experiment: evidence from the first year." The Quarterly Journal of Economics 127 (3):1057-1106.

Gassoumis, Zachary D., Kathryn T. Fike, Anna N. Rahman, Susan M. Enguidanos, and Kathleen H. Wilber. 2013. "Who Transitions to the Community From Nursing Homes? Comparing Patterns and Predictors for Short-Stay and Long-Stay Residents." Home Health Care Services Quarterly 32 (2):75-91.

Grabowski, David C. 2001. "Medicaid Reimbursement and the Quality of Nursing Home Care." Journal of Health Economics 20 (4):549-569. 
Grabowski, David C. and Jonathan Gruber. 2007. "Moral Hazard in Nursing Home Use." Journal of Health Economics 26 (3):560-577.

Grabowski, David C., Jonathan Gruber, and Joseph J. Angelelli. 2008. "Nursing Home Quality as a Common Good." Review of Economics and Statistics 90 (4):754-764.

Hackmann, Martin B. 2017. "Incentivizing Better Quality of Care: The Role of Medicaid and Competition in the Nursing Home Industry." NBER Working Paper 24133.

Hansen, Bruce E. 2017. "Regression kink with an unknown threshold." Journal of Business E3 Economic Statistics 35 (2):228-240.

Ho, Kate and Ariel Pakes. 2014. "Hospital choices, hospital prices, and financial incentives to physicians." American Economic Review 104 (12):3841-84.

Holup, Amanda A., Zachary D. Gassoumis, Kathleen H. Wilber, and Kathryn Hyer. 2016. "Community Discharge of Nursing Home Residents: The Role of Facility Characteristics." Health Services Research 51 (2):651-666.

Intrator, Orna, David C. Grabowski, Jacqueline Zinn, Mark Schleinitz, Zhanlian Feng, Susan Miller, and Vincent Mor. 2007. "Hospitalization of Nursing Home Residents: The Effects of States' Medicaid Payment and Bed-Hold Policies." Health Services Research 42:16511671.

Jin, Ginger Zhe, Ajin Lee, and Susan Feng Lu. 2018. "Medicare Payment to Skilled Nursing Facilities: The Consequences of the Three-Day Rule." NBER Working Paper 25017.

Kaiser Family Foundation. 2015. "Medicaid Home and Community-Based Services Programs: 2012 Data Update." .

Konetzka, R. Tamara, Daifeng He, Jing Guo, and John A. Nyman. 2014. "Moral Hazard and Long-Term Care Insurance." Unpublished manuscript.

Manning, Willard G, Joseph P Newhouse, Naihua Duan, Emmett B Keeler, and Arleen Leibowitz. 1987. "Health insurance and the demand for medical care: evidence from a randomized experiment." The American economic review :251-277.

McGuire, Thomas G. 2011. "Demand for health insurance." In Handbook of health economics, vol. 2. Elsevier, 317-396.

McKnight, Robin. 2006. "Home care reimbursement, long-term care utilization, and health outcomes." Journal of Public Economics 90 (1-2):293-323.

Meador, Rhoda, Emily Chen, Leslie Schultz, Amanda Norton, Charles Henderson Jr., and Karl Pillemer. 2011. "Going Home: Identifying and Overcoming Barriers to Nursing Home Discharge." Care Management Journals 12 (1):2-11.

Mommaerts, Corina. 2017. "Are Coresidence and Nursing Homes Substitutes? Evidence from Medicaid Spend-Down Provisions." Unpublished manuscript.

Mor, Vincent, Jacqueline Zinn, Pedro Gozalo, Zhanlian Feng, Orna Intrator, and David C. 
Grabowski. 2007. "Prospects For Transferring Nursing Home Residents To The Community." Health Affairs 26:1762-1771.

O'Keeffe, Janet. 1999. People With Dementia: Can They Meet Medicaid Level-of-Care Criteria for Admission to Nursing Homes and Home and Community-Based Waiver Programs? AARP Public Policy Institute.

Pipal, William. 2012. "You Don't Have to Go Home But You Can’t Stay Here: The Current State of Federal Nursing Home Involuntary Discharge Laws." Elder Law Journal $20(1): 235-268$.

Rabiner, Donna J, Sally C Stearns, and Elizabeth Mutran. 1994. "The effect of Channeling on in-home utilization and subsequent nursing home care: a simultaneous equation perspective." Health services research 29 (5):605.

Roemer, Milton I. 1961. "Bed supply and hospital utilization: a natural experiment." Hospitals 35 (1):36-42.

Shigeoka, Hitoshi. 2014. "The effect of patient cost sharing on utilization, health, and risk protection." American Economic Review 104 (7):2152-84.

Siegel Bernard, Tara and Robert Pear. 2018. "Nursing Home Evictions Draw U.S. Scrutiny." New York Times Feb 23:B1.

Trottmann, Maria, Peter Zweifel, and Konstantin Beck. 2012. "Supply-side and demand-side cost sharing in deregulated social health insurance: Which is more effective?" Journal of Health Economics 31 (1):231-242.

Weissert, William G. and William J. Scanlon. 1985. "Determinants of Nursing Home Discharge Status." Medical Care 23 (4):333-343. 


\section{For Online Publication}

\section{A Home Health Care Programs}

Home and community based services (HCBS) range from intensive LTC such as round-theclock services and private duty nursing to temporary services including home health aides and help with housekeeping tasks, but may also encompass home-delivered meals, support of informal caregivers, and home accessibility adaptations. States offer various programs covering HCBS in order to reduce their Medicaid LTC spending and to offer eligible seniors more flexibility in meeting their LTC needs. Under waivers based on Section 1915(c) of the Social Security Act, states have developed programs that cover services such as adult day care, assisted living, home modifications, home delivered meals, and respite for informal caregivers. ${ }^{36}$ 1915(c) waivers explicitly require states to demonstrate that the covered HCBS are cheaper to provide than institutional LTC.

In addition, the Money Follows the Person (MFP) demonstration program makes grants available to states to pursue programs that pay for HCBS of previous nursing home residents. ${ }^{37} \mathrm{MFP}$ is therefore closely related to the discharge incentives of existing nursing home residents. By subsidizing HCBS, it makes prolonging a nursing home stay relatively less attractive from the resident's perspective.

Overall, MFP and programs authorized under 1915(c) waivers increase seniors' freedom of choice of LTC arrangements and therefore approximate a policy that provides LTC vouchers. Under a typical voucher program, Medicaid beneficiaries would receive a lump-sum payment that they can spend on their preferred LTC type while incurring the price that a private payer may pay. In practice, seniors covered by Medicaid do not face private fees, but otherwise the existing HCBS programs resemble to a policy that provides LTC vouchers. We simulate nursing home utilization under a voucher program in Section 8.2.

\section{B Details for Theoretical Discussion}

In this section, we describe the key predictions of our model in greater detail. See Section 3 for the model set-up. Specifically, we assume that nursing homes and residents can exert positive levels of effort, $e^{S N F}, e^{r e s} \geq 0$, to increase the probability of a discharge, $D=1$ :

$$
\operatorname{Pr}\left[D=1 \mid e^{S N F}, e^{r e s}\right]=D^{e x o g}+\alpha e^{S N F}+\beta e^{r e s},
$$

\footnotetext{
${ }^{36} \mathrm{See} \quad$ https://www.medicaid.gov/medicaid/hcbs/authorities/1915-c/index.html for details and https://www.cms.gov/Outreach-and-Education/American-Indian-Alaska-Native/AIAN/LTSS-TA-Center/ info/1915-c-waivers-by-state.html for a list of waivers by state.

${ }^{37}$ See https://www.medicaid.gov/medicaid/ltss/money-follows-the-person/index.html for details. We cannot study the effects of MFP because it started in 2009, i.e. after our sample period.
} 
where $D^{\text {exog }}$ denotes an exogenous discharge rate in the absence of any positive effort. We assume that efforts weakly increase the discharge probability: $\alpha, \beta \geq 0$.

\section{B.1 Resident Effort}

Residents trade off the utility from staying an additional week in the nursing home versus returning to the community. We assume the following indirect conditional utility:

$$
W(\tau)= \begin{cases}u-\kappa p^{\tau}+\eta^{S N F} & \text { if stay } \\ \eta^{\text {home }} & \text { otherwise }\end{cases}
$$

where $u$ is the resident's gross utility from nursing home care and we normalize utility from home health care to zero. $\kappa$ is a price coefficient and $p^{\tau}$ is the per-period price paid by the resident (i.e. $p^{M}=0$ ). $\eta^{S N F}$ and $\eta^{\text {home }}$ are type I extreme value taste shocks. Residents prefer to be discharged and hence exert strictly positive effort if $u-\kappa p^{\tau} \leq \eta^{h o m e}-\eta^{S N F}$.

\section{B.2 Provider Effort}

Here, we show that the Medicaid discharge rate increases in the occupancy rate above some occupancy threshold $o c^{*}$ - as shown in Figure 1 in the main text - under simplifying assumptions that yield a closed-form solution. Specifically, we assume that the occupancy rate is fixed, newly admitted residents are private payers, there are no payer type transitions, and the exogenous discharge rate as well as the resident's discharge effort are equal to zero. Hence, a resident is only discharged if the nursing home provides strictly positive effort. The focal bed can either be empty, $\tau=0$, or filled with a private payer or Medicaid beneficiary: $\tau=P, M$. We assume that providers exert discharge effort during the period, but that discharges continue to be stochastic and are realized at the time as new arrivals at the end of the period. We can then define the following Bellman equation:

$$
V(\tau, o c)= \begin{cases}\frac{\Pi(P)}{1-\delta} & \text { if } \tau=P \\ \max _{e \geq 0}\{\Pi(M)-c(e)+D(e) V(0, o c)+(1-D(e)) \delta V(M)\} & \text { if } \tau=M \\ \delta[\phi(o c) V(P, o c)+(1-\phi(o c)) V(0, o c)] & \text { if } \tau=0\end{cases}
$$

where $\Pi(\tau)$ is the payer type-specific per-period profit, $c(e)$ denotes the provider's cost of effort, $D(e)$ is the discharge probability as a function of the SNF's effort, $\phi(o c)$ is the probability of refilling a vacant bed, and $\delta$ is the discount factor. Note that the nursing never has an incentive to discharge a private payer in this model, which leads to the functional form of $V(P, o c)$.

For Medicaid-covered residents, the nursing home has no incentive to exert strictly pos- 
itive effort below occupancy level $o c<o c^{*}$ because the refill probability is too low and the option value of vacating a bed does not compensate for forgone Medicaid profits. Hence, $V(M, o c)=\frac{\Pi(M)}{1-\delta}$ for $o c<o c^{*}$. For $o c \geq o c^{*}$, we have the first order condition:

$$
c^{\prime}(e)=D^{\prime}(e)[V(0, o c)-\delta V(M, o c)]
$$

Assuming $c(e)=e^{2}$ and with $D^{\prime}(e)=\beta$, see equation (B.1), we have

$$
e^{*}=\frac{\beta}{2}[V(0, o c)-\delta V(M, o c)]
$$

and

$$
V(M, o c)=\frac{\Pi(M)-c\left(e^{*}\right)}{1-\delta\left(1-D\left(e^{*}\right)\right)}+\frac{D\left(e^{*}\right)}{1-\delta\left(1-D\left(e^{*}\right)\right)} V(0, o c)
$$

Defining:

$$
F=e^{*}-\frac{\beta}{2}[V(0, o c)-\delta V(M, o c)]=0,
$$

we have $d F / d e^{*}=1$ as $V(M, o c) / d e^{*}=0$ because of the first order condition. We also have

$$
\frac{d F}{d o c}=-\frac{\beta}{2}\left[1-\frac{\delta D\left(e^{*}\right)}{1-\delta\left(1-D\left(e^{*}\right)\right)}\right] \frac{d V(0, o c)}{d o c} .
$$

Since we have $d V(0, o c) / d o c>0$ and $\left[1-\frac{\delta \mu}{1-\delta(1-\mu)}\right]>0$, we get $d F / d o c<0$. This implies $d e^{*} / d o c>0$ based on the implicit function theorem. Hence, provider efforts and consequently Medicaid discharge rates increase in the occupancy rate for $o c \geq o c^{*}$.

\section{Data Appendix}

In this Section, we provide additional information about our data sources and describe the sample in more detail.

The quarterly or more frequent nursing home resident assessment in the MDS provide information on a large number of health measures that cover a variety of cognitive, physical functional, behavioral, communication, and disease-related conditions. We reduce the wealth of health measures to a few key statistics that are commonly used in Medicaid and Medicare reimbursement methodologies. Most importantly, these include the resident's CMI, which is normalized to one and summarizes the expected resource utilization relative to the average resident. We also consider five other health measures that enter into the calculation of the CMI: (i) physical disabilities that are measured by the amount of required help with activities of daily living (ADL) such as toileting or assistance with eating, bed mobility, and transferring, (ii) whether the resident requires clinically complex treatments such as 


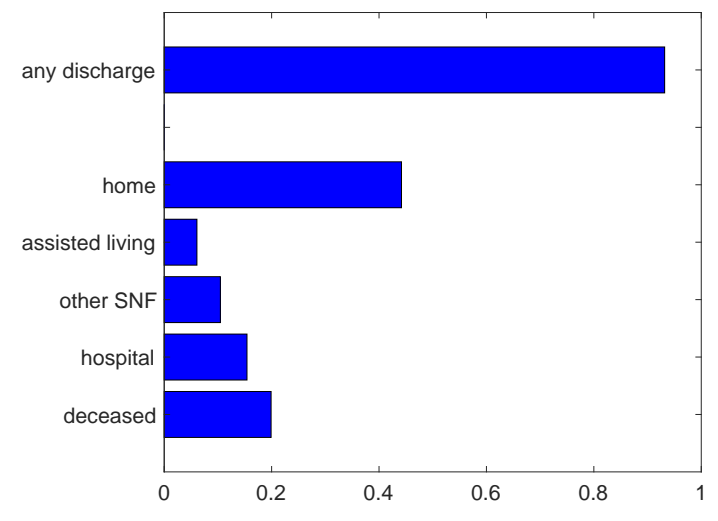

(a) Discharge Probabilties on the Stay Level

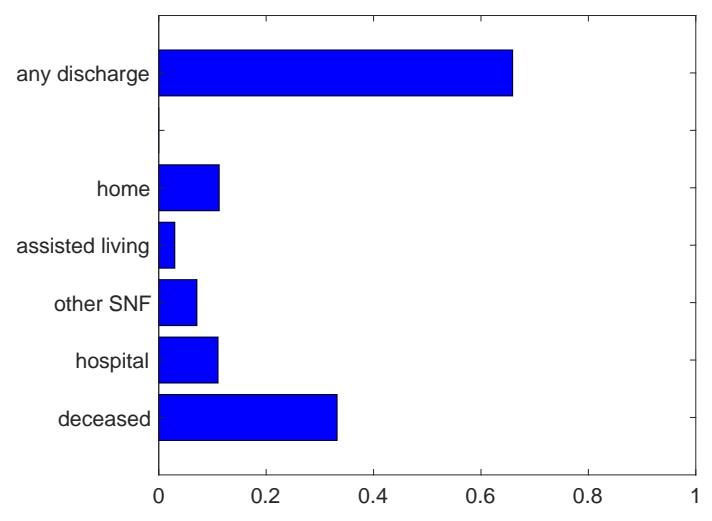

(b) Discharge Probabilities Weighted by Length of Stay

Figure C.1: Discharge Probabilities by Destination

Notes: The figure summarizes the propensity of different discharge reasons. Any discharge is an indicator that turns on if the stay ends with a discharge. The remaining stays are censored. "Home", "Assisted Living", "Other SNF", "Hospital", and "Deceased" are indictor variables that turn on if the stay ends with a discharge to the community, to an assisted living facility, a different SNF, a hospitals or if the resident deceased, respectively. The figures shows the fraction of stays that end accordingly. In the left figure, the unit of observation is the nursing home stay. In the right figure, the unit of observation is week of the nursing home stay.

chemotherapy, oxygen therapy, or dialysis, (iii) depression, (iv) impaired cognition, and (v) behavioral problems.

The MDS also indicates the admission and discharge dates for each resident, which allows us to construct the exact length of each nursing home stay. We observe a discharge code, which provides information on the reason of discharge and the institution the resident was discharged to. Figure C.1a displays stay-level discharge probabilities overall and by destination. In our sample, $93 \%$ of nursing home residents have been discharged by the end of the sample period. Almost half of them return to the community, $20 \%$ of residents pass away in the nursing home, and the remaining residents enter an assisted living facility, another nursing home, or a hospital upon being discharged. When weighting the discharge probability by length of stay, i.e. using week-level discharges, Figure C.1b shows a different pattern. Specifically, we find that only $65 \%$ of residents have been discharged by the end of the sample period. Since residents' health status deteriorates over time, fewer SNF stays end in a home discharge (11\%) and more stays end through the resident's death (33\%) when weighting by the length of stay, thereby explaining the differences between Figures C.1a and C.1b.

To investigate differences in discharge patterns between residents with different payer sources, we combine the MDS with administrative Medicare and Medicaid claims data from 
the MedPAR and MAX databases, respectively. We link the information at the nursing home-stay level and are thus able to quantify the days of each stay covered by Medicare and Medicaid. We assume that the remaining days are paid out-of-pocket, given that only a small fraction of residents have private LTC insurance. Importantly, we observe the exact transition date between private pay and Medicaid eligibility, which is key for our empirical analysis.

To quantify the occupancy rate of each nursing home at any point in time, we combine admission and discharge date information from the MDS with information on the number of licensed beds from the On-Line Survey, Certification, and Reporting system (OSCAR). This survey provides information from state surveys on all federally-certified Medicaid and Medicare nursing homes in the U.S. (see, e.g., Grabowski, 2001). Figure C.2 presents a histogram for the number of licensed beds. While about 30\% of all SNFs have between 100 and 120 beds, there is substantial variation in facility size. Table C.1 summarizes within nursing home week-to-week variation in the occupancy rate. The cells document the relative frequency compared to any transition between $90 \%$ and $100 \%$ occupancy. The cells on the main diagonal sum to $58 \%$ suggesting that occupancy rate changes from week-to-week of one or more percentage points occur $42 \%$ of the time.s

We also use data from distinct nursing home surveys from California and Pennsylvania, which collect information on private and Medicaid rates not included in OSCAR (see Hackmann, 2017). ${ }^{38}$ For California SNFs, we infer daily private and Medicaid rates by dividing annual revenue by the number of resident-days for each payer type. The average daily private rate amounts to $\$ 218$ and $\$ 158$ in Pennsylvania and California, respectively, and the corresponding average Medicaid rates are $\$ 188$ and $\$ 127$. These differences indicate that the profitability varies by payer types, a fact that we leverage in some of our empirical analyses.

To investigate potential differences in access to community-based care further, we supplement Table 1 with data from two surveys. First, we select respondents from the HRS who have spent at least one night in a nursing home in the two years prior to a given survey wave. Table C.2 shows selected summary statistics by payer type. ${ }^{39}$ We first consider the role of respondents' children as potential informal caregivers. The fractions of respondents who receive help with ADL or IADL are roughly similar across payer types. Private payers expect more help in the future, but Medicaid beneficiaries are more likely to receive financial

\footnotetext{
${ }^{38}$ The Pennsylvania survey data were provided by the Bureau of Health Statistics and Research of the Pennsylvania Department of Health. California data come from the Office of Statewide Health Planning and Development (see http://www.oshpd.ca.gov/HID/Products/LTC/AnnFinanclData/PivotProfls/default.asp).

${ }^{39} \mathrm{HRS}$ respondents are designated as Medicaid beneficiaries if they report being covered by a Medicaid plan. We define them as private payers if their nursing home stay was not covered any type of government plan (Medicaid or Medicare) or if they hold private long term care insurance.
} 


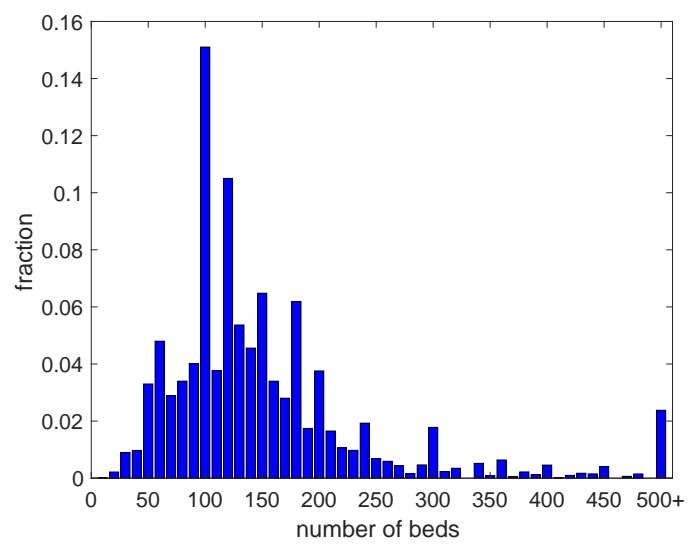

Notes: The figure presents a histogram of the overall number of licensed beds. The unit of observation is the week of the nursing home stay.

Figure C.2: Number of Licensed Beds

Table C.1: Week-to-Week Transitions in Occupancy 90\%-100\%

\begin{tabular}{|c|ccccccccccc|}
\hline & $90 \%$ & $91 \%$ & $92 \%$ & $93 \%$ & $94 \%$ & $95 \%$ & $96 \%$ & $97 \%$ & $98 \%$ & $99 \%$ & $100 \%$ \\
\hline $90 \%$ & $3.51 \%$ & $0.93 \%$ & $0.63 \%$ & $0.36 \%$ & $0.16 \%$ & $0.08 \%$ & $0.04 \%$ & $0.02 \%$ & $0.01 \%$ & $0.00 \%$ & $0.00 \%$ \\
$91 \%$ & $0.77 \%$ & $3.75 \%$ & $1.05 \%$ & $0.73 \%$ & $0.37 \%$ & $0.18 \%$ & $0.07 \%$ & $0.03 \%$ & $0.01 \%$ & $0.00 \%$ & $0.00 \%$ \\
$92 \%$ & $0.49 \%$ & $0.83 \%$ & $4.55 \%$ & $1.24 \%$ & $0.71 \%$ & $0.40 \%$ & $0.17 \%$ & $0.08 \%$ & $0.03 \%$ & $0.01 \%$ & $0.00 \%$ \\
$93 \%$ & $0.31 \%$ & $0.57 \%$ & $1.02 \%$ & $5.48 \%$ & $1.32 \%$ & $0.86 \%$ & $0.39 \%$ & $0.19 \%$ & $0.06 \%$ & $0.02 \%$ & $0.00 \%$ \\
$94 \%$ & $0.17 \%$ & $0.34 \%$ & $0.59 \%$ & $1.08 \%$ & $5.66 \%$ & $1.46 \%$ & $0.84 \%$ & $0.36 \%$ & $0.14 \%$ & $0.03 \%$ & $0.01 \%$ \\
$95 \%$ & $0.11 \%$ & $0.19 \%$ & $0.35 \%$ & $0.70 \%$ & $1.22 \%$ & $6.35 \%$ & $1.55 \%$ & $0.89 \%$ & $0.34 \%$ & $0.08 \%$ & $0.02 \%$ \\
$96 \%$ & $0.05 \%$ & $0.09 \%$ & $0.18 \%$ & $0.36 \%$ & $0.70 \%$ & $1.33 \%$ & $7.18 \%$ & $1.54 \%$ & $0.79 \%$ & $0.21 \%$ & $0.03 \%$ \\
$97 \%$ & $0.03 \%$ & $0.04 \%$ & $0.09 \%$ & $0.21 \%$ & $0.37 \%$ & $0.74 \%$ & $1.29 \%$ & $7.47 \%$ & $1.61 \%$ & $0.47 \%$ & $0.07 \%$ \\
$98 \%$ & $0.02 \%$ & $0.02 \%$ & $0.05 \%$ & $0.10 \%$ & $0.17 \%$ & $0.34 \%$ & $0.72 \%$ & $1.35 \%$ & $8.45 \%$ & $1.13 \%$ & $0.14 \%$ \\
$99 \%$ & $0.00 \%$ & $0.01 \%$ & $0.01 \%$ & $0.03 \%$ & $0.04 \%$ & $0.10 \%$ & $0.22 \%$ & $0.46 \%$ & $1.03 \%$ & $5.14 \%$ & $0.28 \%$ \\
$100 \%$ & $0.00 \%$ & $0.00 \%$ & $0.00 \%$ & $0.01 \%$ & $0.01 \%$ & $0.02 \%$ & $0.04 \%$ & $0.06 \%$ & $0.14 \%$ & $0.26 \%$ & $0.87 \%$ \\
\hline
\end{tabular}

Notes: The table summarizes within nursing home week-to-week variation in the occupancy rate. Current and next week's occupancy rate are displayed in rows and columns, respectively. Each cell documents the relative frequency, compared to any transition between 90 and 100\% occupacny. For expositional reasons, occupancy variation is only shown between $90 \%$ and $100 \%$. 
Table C.2: Summary Statistics: Informal Caregivers, Income, and Asset (HRS)

\begin{tabular}{|c|c|c|c|c|}
\hline & \multicolumn{2}{|c|}{ Medicaid } & \multicolumn{2}{|c|}{ Private } \\
\hline & Mean & SD & Mean & SD \\
\hline Kids help with ADL & 0.203 & $(0.402)$ & 0.131 & $(0.338)$ \\
\hline Kids help with IADL & 0.157 & $(0.364)$ & 0.144 & $(0.351)$ \\
\hline Kids will help in future & 0.0433 & $(0.204)$ & 0.141 & $(0.348)$ \\
\hline Kids help with health care cost & 0.0777 & $(0.268)$ & 0.0279 & $(0.165)$ \\
\hline Hours per month kids helping & 30.11 & (99.79) & 21.67 & (80.95) \\
\hline \multirow[t]{2}{*}{ Home ownership } & 0.274 & $(0.446)$ & 0.597 & $(0.491)$ \\
\hline & \multicolumn{2}{|c|}{$\begin{array}{l}\text { Mean } \\
\quad[\mathrm{p} 25, \mathrm{p} 50, \mathrm{p} 75]\end{array}$} & \multicolumn{2}{|c|}{$[\mathrm{p} 25, \mathrm{p} 50, \mathrm{p} 75]$} \\
\hline Social security income & \multicolumn{2}{|c|}{$[4704.8,6891.8,9508.0]$} & \multicolumn{2}{|c|}{$[2861.1,6528.9,9199.1]$} \\
\hline Total household income & \multicolumn{2}{|c|}{$\begin{array}{l}11933.6 \quad(11235.4) \\
{[6718.3,9235.1,13703.3]}\end{array}$} & \multicolumn{2}{|c|}{$\begin{array}{cc}19214.1 & (10292.3) \\
{[11271.1,} & 17718.8,26515.2]\end{array}$} \\
\hline Net financial assets & \multicolumn{2}{|c|}{$\begin{array}{c}5495.2 \quad(51800.3) \\
{[0,0,854.2]}\end{array}$} & \multicolumn{2}{|c|}{$\begin{array}{cc}27845.8 & (45702.0) \\
{[0,4750.8,50015.1]}\end{array}$} \\
\hline Total wealth & \multicolumn{2}{|c|}{$\begin{array}{c}32989.8 \quad(119989.1) \\
{[0,394.9,15846.6]}\end{array}$} & \multicolumn{2}{|c|}{$\begin{array}{l}130945.6 \quad(142362.5) \\
{[3316.8,81361.9,237699.4]}\end{array}$} \\
\hline Observations & \multicolumn{2}{|c|}{1,693} & \multicolumn{2}{|c|}{526} \\
\hline
\end{tabular}

Note: All income and asset amounts in 2000 dollars.

Source: Health and Retirement Study 1998 to 2010. 
Table C.3: Summary Statistics: Monthly Income and Assets (NLTCS)

\begin{tabular}{|c|c|c|c|c|c|c|c|}
\hline & \multicolumn{2}{|c|}{$\begin{array}{c}(1) \\
\text { Mcd/Mcd }\end{array}$} & \multicolumn{2}{|c|}{$\begin{array}{c}(2) \\
\text { Prv/Mcd }\end{array}$} & \multicolumn{2}{|c|}{$\begin{array}{c}(3) \\
\operatorname{Prv} / \operatorname{Prv}\end{array}$} & \multirow{2}{*}{$\begin{array}{c}(4) \\
p \text {-value }\end{array}$} \\
\hline & Mean & $\mathrm{SD}$ & Mean & $\mathrm{SD}$ & Mean & $\mathrm{SD}$ & \\
\hline Total income & 622.6 & $(582.6)$ & 792.2 & $(585.7)$ & 1117.3 & $(1516.3)$ & 0.0518 \\
\hline Social Security benefits & 620.8 & $(367.3)$ & 770.7 & $(319.6)$ & 872.0 & (545.1) & 0.1190 \\
\hline Other retirement income & 60.33 & $(203.1)$ & 153.0 & $(361.0)$ & 337.9 & $(875.8)$ & 0.0636 \\
\hline SSI & 10.94 & $(125.7)$ & 0 & (0) & 10.88 & $(101.0)$ & 0.4396 \\
\hline Home ownership & 0.0819 & $(0.280)$ & 0.0899 & $(0.295)$ & 0.160 & $(0.376)$ & 0.2933 \\
\hline Net home value & 5264.2 & $(25189.0)$ & 3995.2 & $(18530.6)$ & 23287.9 & $(115402.0)$ & 0.2368 \\
\hline Observations & & 203 & & 48 & & 66 & \\
\hline
\end{tabular}

Notes: p-value from the $F$-test of the null hypothesis $H_{0}: \beta_{P}=\beta_{M}$ in the regression $Y_{i}=\beta_{0}+\beta_{P}$ Prv $v_{i}+$ $\beta_{M} M c d_{i}+\sum_{t} \delta_{t} \mathbf{1}\left\{L O S_{i}=t\right\}+\epsilon_{i}$, where Prv $v_{i}$ and $M c d_{i}$ are indicators for payer type at the interview, given that payer type at admission is private (excluded category is Medicaid at admission). All amounts in 2004 dollars.

Source: National Long Term Care Survey 1999 and 2004.

support to pay for medical bills and receive more help at the intensive margin. Overall, there are no systematic differences in informal care.

Second, we consider nursing home residents' income and assets. Using data from the HRS again, the bottom part of Table C.2 indicates that private payers have higher income and asset levels than Medicaid beneficiaries and are more likely to own a home. These differences are less substantial, however, when taking into account the cost of nursing home care. The income difference at the median would pay for a nursing home stay of about one month. In practice, most private payers would pay down their assets. At median asset levels, they could afford about one year of SNF care before qualifying for Medicaid. The comparison between private and Medicaid residents in Table C.2 does not reflect the income and asset distributions in our MDS sample because the HRS sample includes SNF residents who qualify for Medicaid at the beginning of their stay, thereby biasing the income and asset measures downward. In our MDS sample, Medicaid and private resident are therefore more homogenous with respect to income and assets than in the HRS.

Using data from the NLTCS of 1999 and 2004, we can construct a sample that is more comparable to the MDS. In contrast to the HRS, the NLTCS samples individuals who are currently residing in a nursing home. Moreover, the NLTCS contains information on the payer type at admission and at the time of the interview, so it allows us to observe payer type switches. Table C.3 shows nursing home residents' average income and assets by payer type including residents who switch from private to Medicaid during their nursing home 
stays. Since we drop residents who are covered by Medicaid at admission in our main analysis, we are particularly interested in any differences between column (2) and (3) in Table C.3. The length of stay adjusted $p$-values in column (4) show that none of the income and asset measures are statistically significantly different at the $95 \%$ level between payer types. However, we note that the averages point to higher home ownership rates among private payers. We revisit the effect of remaining differences between payer types on our main findings in Section 6.2.

\section{Robustness and Additional Empirical Results}

\section{D.1 Kink Point Estimation}

We follow the method proposed by Hansen (2017) to estimate the kink points, i.e. the occupancy rate where nursing homes exert strictly positive effort to discharge Medicaid residents, displayed in Table 2. Specifically, we use the estimated Medicaid discharge profile shown in Figure $3, D_{k}^{M}, k=75, \ldots, 100$, as a our data and estimate piecewise linear regressions of the form

$$
D_{k}^{M}=\beta_{1} \min \left\{k-o c c^{*}, 0\right\}+\beta_{2} \max \left\{k-o c c^{*}, 0\right\}+e_{k},
$$

where $k$ denotes the occupancy rate, occ* is the kink point where the slope of the Medicaid discharge profile changes from $\beta_{1}$ to $\beta_{2}$, and $e_{k}$ is an i.i.d. error term. We estimate occ ${ }^{*}$ by running regression (D.1) for all candidate $o c c^{*}=76, \ldots, 99$ and, for each one, calculating the least squares criterion

$$
\begin{aligned}
& S\left(\hat{\beta}_{1}\left(o c c^{*}\right), \hat{\beta}_{2}\left(o c c^{*}\right), o c c^{*}\right)= \\
& \frac{1}{26} \sum_{k=75}^{100}\left(D_{k}^{M}-\hat{\beta}_{1}\left(o c c^{*}\right) \min \left\{k-o c c^{*}, 0\right\}-\hat{\beta}_{2}\left(o c c^{*}\right) \max \left\{k-o c c^{*}, 0\right\}\right)^{2}
\end{aligned}
$$

where $\hat{\beta}_{1}\left(o c c^{*}\right)$ and $\hat{\beta}_{2}\left(o c c^{*}\right)$ are OLS coefficients estimated conditional on $o c c^{*}$. We then obtain the estimated kink point $\widehat{o c c}^{*}$ as the $o c c^{*}$ that minimizes the least squares criterion (D.2).

\section{D.2 Discharge Patterns to Other Destinations}

We have argued above that financial discharge incentives of providers and patients are most likely to operate in the case of residents who ultimately return to the community. The results shown in Figure 3 confirm this hypothesis. Here, we estimate regression (2) with indicators for different types of discharges as the dependent variable to verify that discharges that are less likely to be affected by financial incentives do not exhibit the same pattern. Figure D.1a 


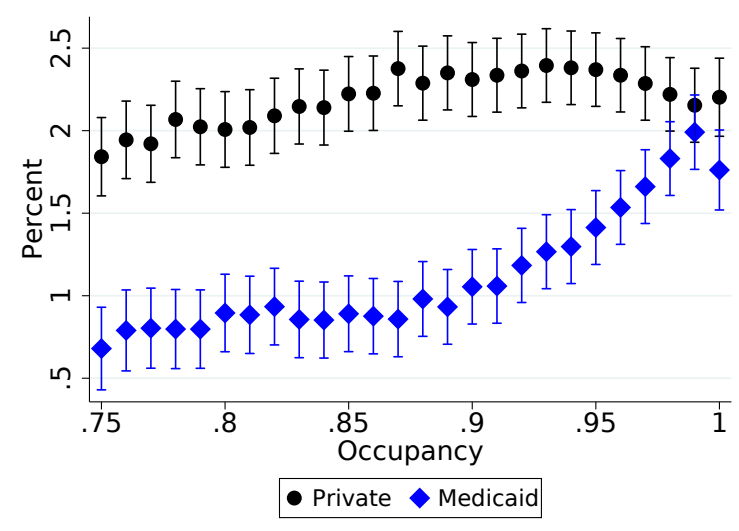

(a) Discharged Home

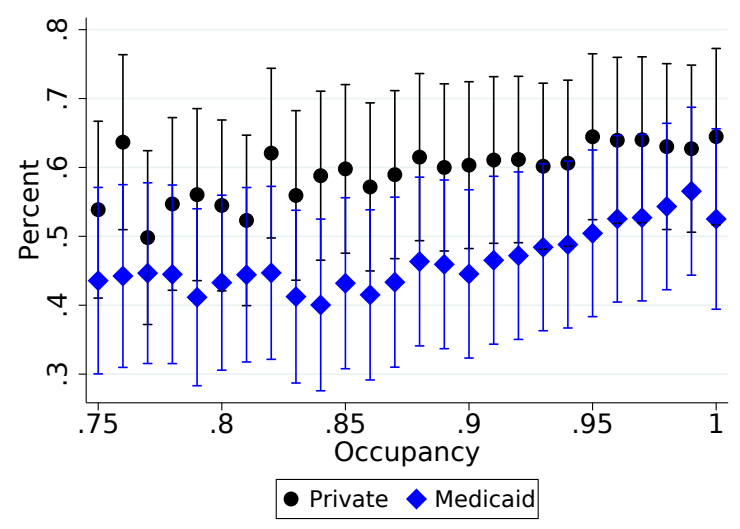

(c) Discharged to Another Nursing Home

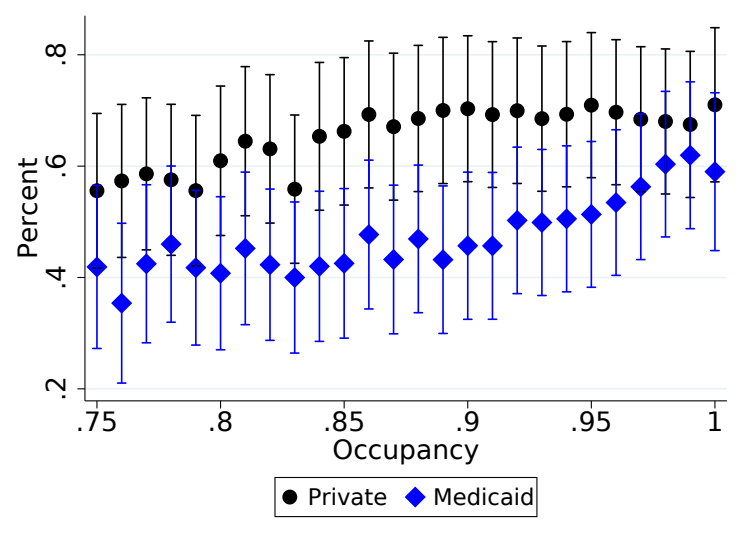

(b) Hospitalized

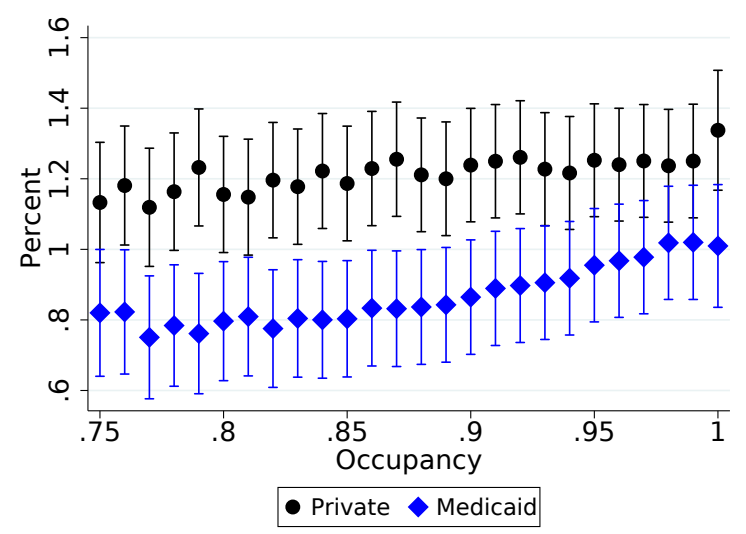

(d) Deceased

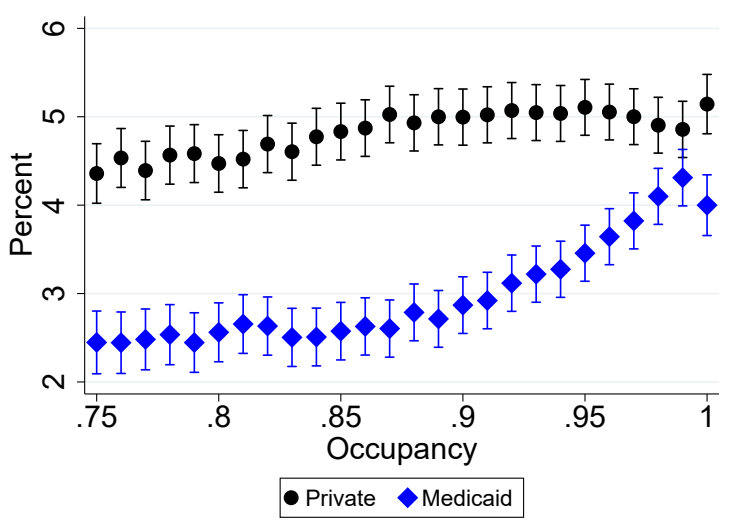

(e) Discharged to Any Destination

Notes: See notes for Figure 3. The dependent variables are indicator variables that equal one if a resident was discharged to the community (Figure D.1a), to a hospital (Figure D.1b), to a different nursing home (Figure D.1c), deceased (Figure D.1d), or discharged overall (any of the above, Figure D.1e) in a given week. The vertical bars indicate $90 \%$ confidence intervals.

Figure D.1: Discharge Rates to Different Destinations by Occupancy and Payer Type 
repeats the results from Figure 3.

Compared to these home discharge patterns, we observe smaller differences between private and Medicaid residents in discharges to a hospital, another nursing home, and due to the resident's death in Figures D.1b, D.1c, and D.1d, respectively. While private payers are discharged to all destinations more frequently, we find that the difference to Medicaid discharge rates amounts to less than half a percentage point. In contrast, home discharge rates differ by over one percentage point at low occupancy rates. We also note that the patterns for discharges to a hospital, another nursing home, and due to the resident's death exhibit a kink for Medicaid residents around an occupancy rate of 90\%. These kinks are much less pronounced than for home discharge rates, however, and are likely due to advantageous selection. Since mostly healthy Medicaid beneficiaries are discharged back to the community at high discharge rates, the Medicaid residents who remain in the nursing home are less healthy and therefore more likely to be hospitalized and to die, see also Section 6.4 and Section D.3 below. The overall discharge patterns in Figure D.1e are similar to home discharge rates, but from Figures D.1a to D.1d it is clear that the sharp increase in Medicaid discharges above $89 \%$ occupancy is primarily driven by discharges to the community.

\section{D.3 Discharge Rates Among Low-Care Residents}

To further verify that Medicaid residents with high LTC needs are not the ones who are discharged at high occupancies, we consider a homogenous subsample of nursing home residents with very little LTC needs. Thereby, we can further isolate the effect financial incentives on nursing home utilization. To identify nursing home residents who have low LTC needs, we follow the methodology developed by Mor et al. (2007) who define residents belonging to a broad category of low care need as those who do not require physical assistance in four ADLs (bed mobility, transferring, using the toilet, and eating) and are not classified as "special rehabilitation" or "clinically complex" residents. Residents who fall into this category have very low care needs and should not receive care in a SNF from a medical point of view but rather use home health care. If we nevertheless observe these patients in a nursing home, they should have very short lengths of stay and therefore high discharge rates. Hence, low discharge rates among low-care Medicaid residents point to overuse of LTC.

Figure D.2 shows three key discharge outcomes for the low-care sample on the left and for comparison purposes for the whole sample on the right: discharges overall and community discharges with and without home health care. First, overall discharge rates among lowcare residents are almost three times as high as for the whole sample reflecting the fact

that the low-care residents have shorter stays, as indicated in the first row. Second, the community discharge rates for private payers in the low-care sample are substantially higher 

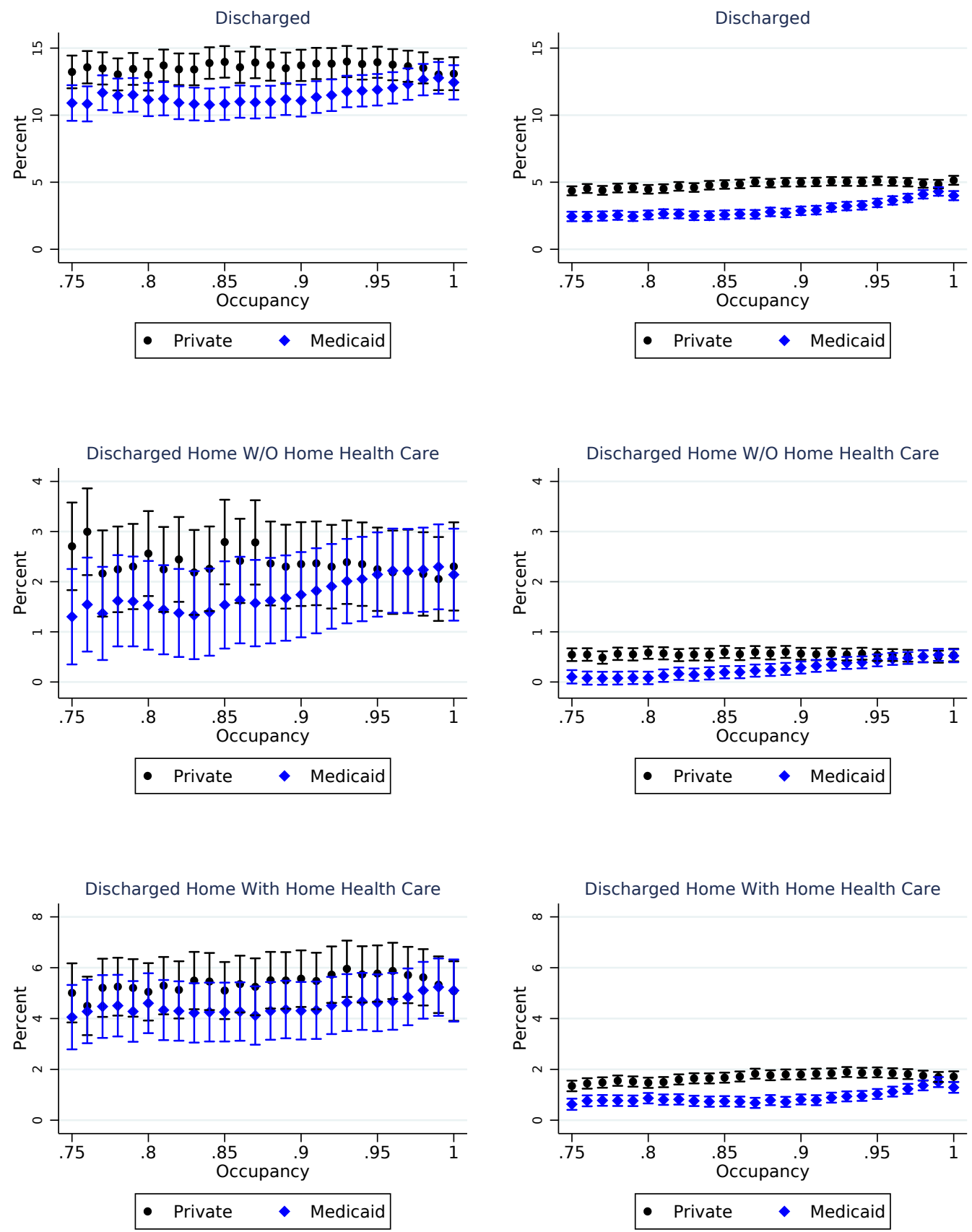

Notes: See notes for Figure 3. This figure compares overall discharges (first row), home discharges without home health care (middle row), home discharges with home health care (bottom row) between resident groups. In the left figures, we focus on residents with low care needs and the right graphs we present the evidence for the baseline sample. The vertical bars indicate $90 \%$ confidence intervals.

Figure D.2: Discharge Rates Among Low Care Need Residents and All Residents 
than for Medicaid beneficiaries at low occupancy rates, see the middle and the bottom left figures. This is particularly the case for residents who do not need LTC in the community, see the middle graph. This result provides further evidence that longer stays of Medicaid beneficiaries contribute to LTC overspending since differences in unobserved health status correlated with payer type are unlikely to explain the observed difference in discharge rates given a highly selected sample with homogenous health profiles. Compared to the discharge differences at low occupancy rates, we find an increase in Medicaid discharge rates at higher occupancies, suggesting again that both consumer and provider profit incentives contribute to longer stays among residents who have very low care needs and should not receive care in a SNF from a medical point of view.

\section{D.4 Effect of Occupancy Rates and Payer Type on Health Out- comes}

Next, we provide evidence on selection on health types by analyzing how current health profiles vary with occupancy. We can thereby test if systematic differences in health between payer types and across occupancies may lead to the observed discharge patterns in Figure 3. Specifically, if Medicaid residents are healthier at higher occupancy rates they may be discharged disproportionately not due to financial incentives of the SNF, but rather because they have lower LTC needs. To test for selection based on health, we estimate regression (2) with the respective health outcome as the dependent variable. The main difference to the results from the analysis shown in Figure 3 is that we do not control for the health profile at admission in this analysis. We consider six health outcomes from residents' most recent assessment: the CMI, ADL limitations, and indicators for clinical complexity, depression, impaired cognition, and behavioral problems. For all health outcomes, a higher value implies that the resident is less healthy.

The top-left panel in Figure D.3 shows that private payers are sicker than Medicaid beneficiaries across the entire occupancy range according to the CMI, a summary measure, conditional on nursing home-year and week-of-stay fixed effects. The CMI differs by less than one tenth of a standard deviation between payer types and across all occupancy rates (see Table 1 for summary statistics of the CMI and other health measures). The graph also indicates that the CMI is slightly decreasing in occupancy for both private and Medicaid residents, i.e. residents are on average slightly healthier at higher occupancy rates. Overall, the CMI trends look very similar between payer types. The downward trend flattens out for Medicaid payers at about $90 \%$ occupancy, which indicates that disproportionately healthier Medicaid residents remain in the nursing home, when compared to private payers, at high occupancies. 

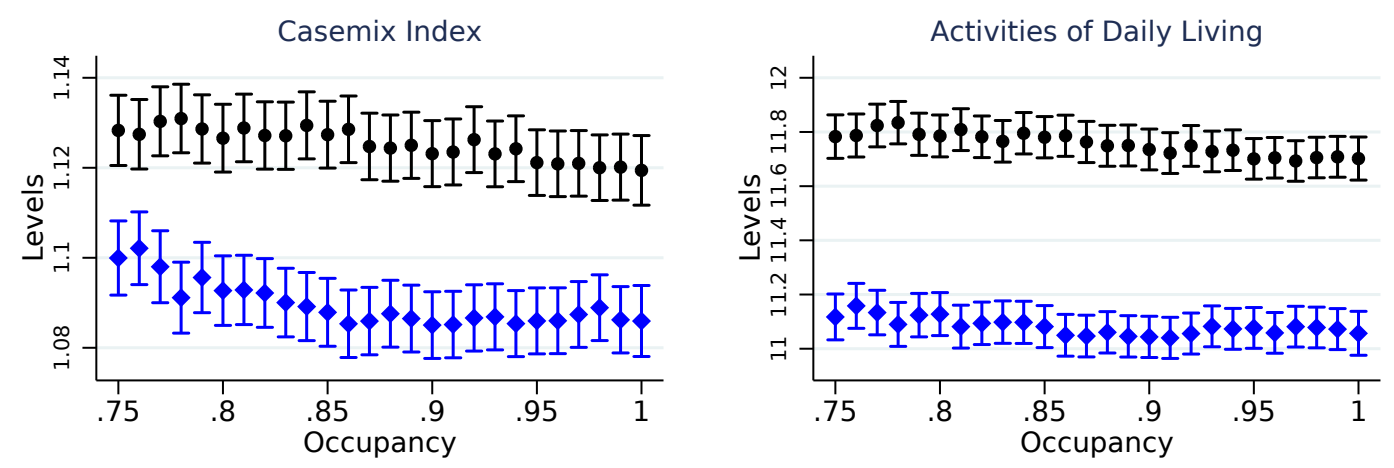

- Private $\diamond$ Medicaid

- Private $\bullet$ Medicaid
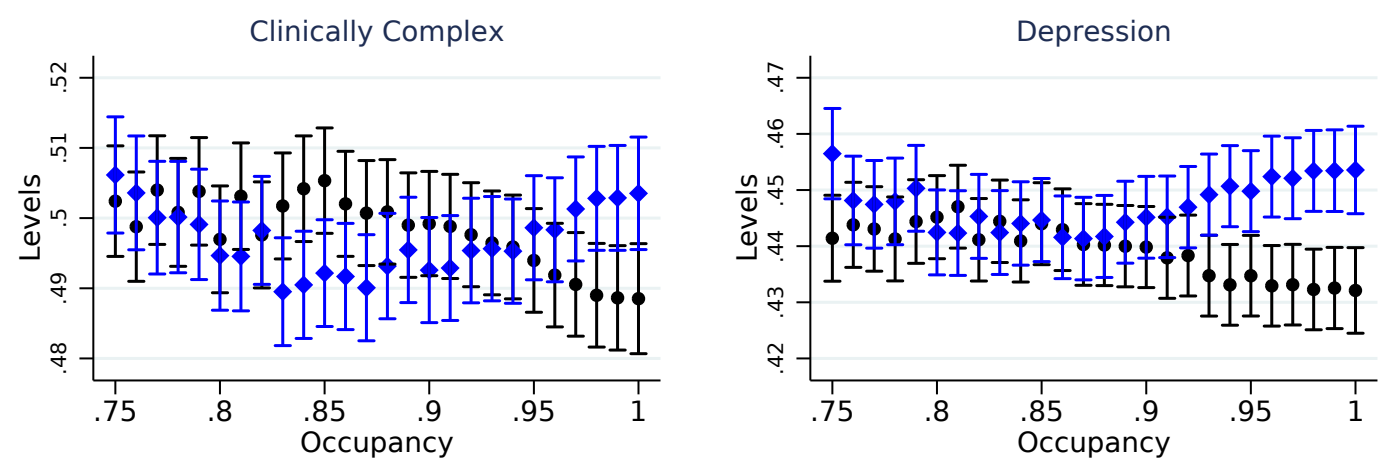

- Private $\diamond$ Medicaid

- Private $\diamond$ Medicaid

Impaired Cognition
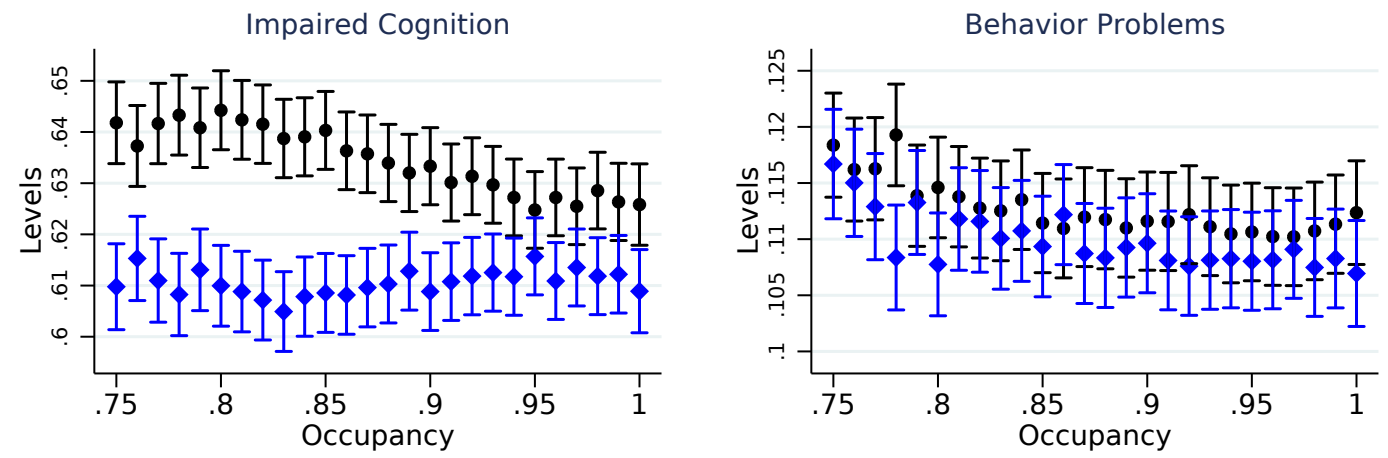

- Private $\bullet$ Medicaid

- Private $\bullet$ Medicaid

Notes: See notes for Figure 3. This figure compares the most recent health profile at the nursing home week level between payer types and occupancy rates. The resident's health status is decreasing in each health measure. The CMI is a summary measure of long term care needs, calculated based on methodology 5.01, and normalized to 1 . The remaining health measures are direct inputs to the CMI formula and provide more granular information on cognitive and physical disabilities. The vertical bars indicate $90 \%$ confidence intervals.

Figure D.3: Most Recent Health Profiles by Payer Type and Occupancy 
In addition to the CMI as a summary measure, we test for selection based on health type for five specific health outcomes. The remaining panels in Figure D.3 do not show many systematic changes in health outcomes as occupancy rates increase that would indicate selection based on health. For clinical complexity, depression, and impaired cognition, the health difference between private and Medicaid residents declines at higher occupancy rates, and Medicaid beneficiaries are less healthy at the highest occupancy rates when it comes to clinical complexity and depression. The health status differences do not exceed $5 \%$ of a standard deviation, however. Overall, we find some evidence for selective discharges based on health types but, the evidence is not as striking as the results on selection on payer type presented in Section 6.1, which suggests that mostly healthy Medicaid beneficiaries are discharged to the community.

\section{D.5 Patient Incentives: Propensity Score Analysis}

In this section, we revisit the baseline estimates in a population of private payers and Medicaid beneficiaries that is even more homogenous than in our baseline results. To this end, we a adopt a propensity score weighting approach. We first estimate the probability of being Medicaid eligible in the given week of the nursing home stay using a flexible logit model that conditions on the highest education level, race, and zip code of former residence fixed effects. All right hand side characteristics remain unchanged during the nursing home stay. Therefore, the predicted probability $\hat{p}_{i}$ does not vary across weeks of the stay. The logit model yields delivers an $R^{2}$ of $7.2 \%$. For Medicaid beneficiaries, we find the following percentiles for the predicted probabilities. At the 10th percentile, we find a Medicaid probability of $29 \%$. This probability increases to $45 \%$ and $61 \%$ at the 50 th and the 90 th percentile, respectively. For private payers, the Medicaid probabilities increase from $20 \%$ to $39 \%$ and $54 \%$ at the 10th, the 50th, and the 90th percentile, respectively. The overlap between the two predicted discharge distributions is therefore very good, suggesting that an inverse propensity score weighting approach yields a relatively small bias compared to other matching algorithms (Busso, DiNardo, and McCrary, 2014).

We then weight Medicaid observations by 1 and assign a weight of $\frac{\hat{p}_{i}}{1-\hat{p}_{i}}$ to private payer observations. Intuitively, we assign private payers a higher weight if they are more likely to transition into Medicaid conditional on observables. We then estimate equation (2) using these regression weights. Our findings are summarized in Figure D.4, which are very similar to our baseline findings presented in Figure 3. Since Medicaid-covered and private residents are balanced based on observables in this result, we can further isolate the role of residents' financial incentives in determining discharge rates. Specifically, we find a similar gap between discharge rates at low occupancies as in Figure 3, suggesting that this difference is indeed 


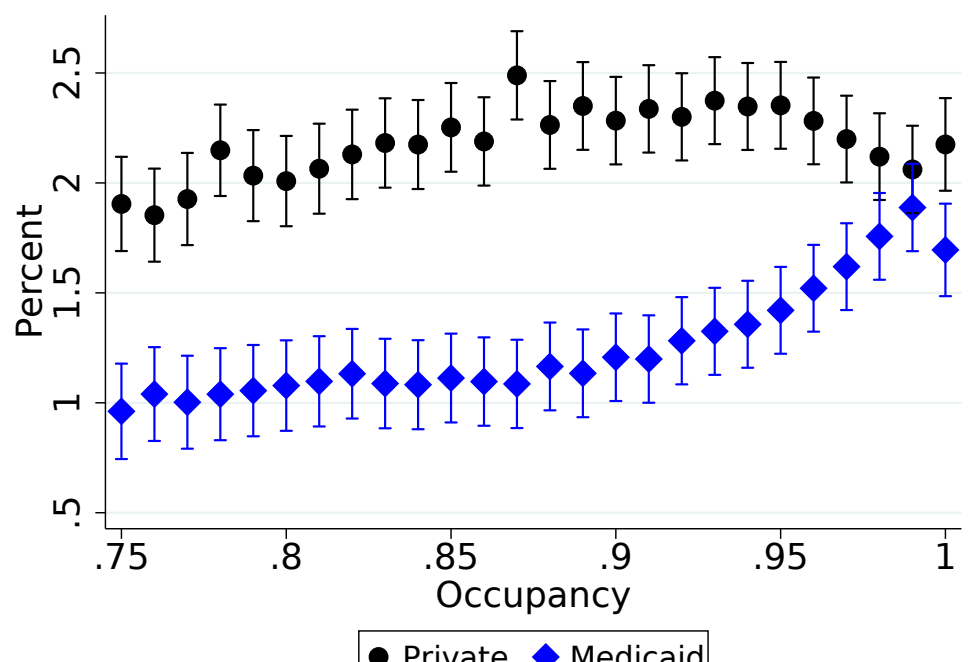

- Private Medicaid

Notes: See notes for Figure 3. The dependent variables are indicator variables that equal one if a resident was discharged to the community. The underlying regression model, see equation (2), is weighted by a propensity score of Medicaid eligibility. The vertical bars indicate 90\% confidence intervals.

Figure D.4: Home Discharges: Propensity Score Weighted

driven by patient moral hazard.

\section{D.6 Patient Incentives: Medicaid Applications}

In this section, we revisit the effect of resident financial incentives on the home discharge probability among Medicaid applicants. In the MDS assessment data, we are able to infer whether a resident has applied for Medicaid coverage and is waiting on a final decision. We restrict the sample to this subsample of residents and focus on the weeks following the assessment date when a pending Medicaid application was first indicated. This sample refinement mitigates remaining differences between private payers and Medicaid. By comparing residents who have applied for Medicaid and whose application has or has not yet been approved, we effectively condition on the residents' level of assets since only residents with resources close to the Medicaid eligibility threshold would consider applying for benefits. Hence, the application outcome only affects home discharge rates through changes in financial incentives.

Estimating regression (D.3) on the subsample of Medicaid applicants, we find a reduction in the home discharge rates among Medicaid beneficiaries of 0.5 percentage points, $\hat{\delta}=$ -0.005 (S.E. 0.01 percentage points). This estimate is slightly smaller in magnitude than the average difference in home discharge rates presented in Figure 3. Nevertheless, the point estimate confirms that financial resident incentives affect the length of stay. 


\section{D.7 Patient Incentives: Border Analysis}

In this section, we exploit differences in Medicaid eligibility criteria between states in a border analysis. In Pennsylvania, Medicaid beneficiaries can retain higher asset levels $(\$ 2,400$ to $\$ 8,000$ ) when compared to Ohio (a most \$1,500). ${ }^{40}$ Furthermore, Pennsylvania permits the spouse living in the community to retain a larger set of the couple's assets when considering the eligibility for Medicaid. In particular, 401k and other retirement plans of the community spouse are not counted as resources for purposes of the institutionalized spouse qualifying for Medicaid in Pennsylvania, but they are in Ohio and New Jersey. Finally, Ohio has a more aggressive estate recovery program which looks to the Medicaid recipient's assets to reimburse the state for the money spent on the individual's care provided in the nursing home.

These rules indicate that Medicaid eligibility standards are more generous in Pennsylvania than in the neighboring states of Ohio and New Jersey. All else equal, we therefore expect a larger fraction of nursing home residents in Pennsylvania to be on Medicaid. To isolate this source of variation, we conduct a state border analysis and restrict the sample to seniors who resided in counties on the Ohio-Pennsylvania border or the Pennsylvania-New Jersey border prior to their nursing home stay. We then revise the baseline regression as follows:

$$
Y_{i j s t}=\delta \times \text { Mcaid }_{i s}+\sum_{k=75}^{100} \gamma^{k} \text { occ }_{j t-1}^{k}+\text { border }_{i}+\alpha_{c}+\alpha_{s}+X_{i}^{\prime} \beta+\epsilon_{i j s t}
$$

For simplicity, we have omitted the full sequence of interaction terms between the occupancy rate fixed effects and Medicaid eligibility. Instead, we include only the Medicaid indicator, whereby $\delta$ denotes the average difference in weekly home discharge rate between payer types. We also drop nursing-home year fixed effects as we exploit cross-sectional variation between counties on either side of the border. Instead, we only control for a state-border fixed effect, $\operatorname{border}_{i}$. To isolate the variation in Medicaid eligibility between states, we instrument for Mcaid $_{\text {is }}$ using a Pennsylvania and a New Jersey state indicator variable (Ohio is the omitted category).

Our first stage parameters of interest equal 0.043 (S.E. 0.0013) for the Pennsylvania indicator and -0.023 (S.E. 0.003) for the New Jersey indicator. These estimates suggests that residents residing in Pennsylvania are more likely to transition into Medicaid coverage when compared to residents from New Jersey or Ohio. Specifically, the probability of transitioning into Medicaid in Pennsylvania's bordering counties, bordering Ohio, exceeds the probability

\footnotetext{
${ }^{40}$ See http://hartleelderlaw.com/download/i/mark_dl/u/1038829/15106695/Ohio and PA Medicaid Differences.pdf and http://www.state.nj.us/humanservices/dmahs/clients/medicaid/medicaid_program_eligibility.pdf.
} 
in Ohio's bordering counties by 4.3 percentage points. The negative coefficient on the New Jersey indicator suggests that this difference is more pronounced at the Pennsylvania-New Jersey border. The IV estimate for $\delta$ in regression (D.3) equals -0.009 (S.E. 0.006). This estimate suggests that the discharge rate falls by 0.9 percentage points as seniors transition into Medicaid. This difference is similar to the average difference in discharge profiles presented in Figure 3. However, due to the additional variation introduced by the IV, the point estimate is not significant at the $10 \%$ level with a $t$-statistic of 1.5 . Nevertheless, this result supports the conclusion that differences in discharge rates are partly driven by patient incentives.

\section{D.8 Medicare Bunching}

In this section, we exploit a sharp discontinuity in co-pay for Medicare beneficiaries to provide further evidence that patient incentives affect the length of nursing home stays. Starting on the 21st day of a Medicare benefit episode, residents are required to pay a daily co-pay rate of about $\$ 150$ in the relevant sample period. ${ }^{41}$ The benefit period can encompass multiple nursing home stays as long as the break between stays is less than 60 calendar days in which case the co-pay discontinuity applies to the cumulative number of days across stays. For example, suppose a Medicare beneficiary is discharged on the 10th day and then returning to a nursing home 40 days later. Then the senior faces the daily co-pay starting on the 11th day of this nursing home stay.

We exploit the cumulative nature of Medicare coverage between stays in our empirical analysis, which allows us to control for day of stay fixed effects. Specifically, we again convert the data to a long format and analyse nursing home disccharges on a daily basis. We then test for bunching in the discharge timing aournd the discontinuity using the following event study regression framework:

$$
Y_{i j d t}=\sum_{k=-19}^{19} \gamma^{k} \times \mathbf{1}\{\text { Days until Copay }=k\}+\alpha_{d}+\alpha_{d o w}+\epsilon_{i j d t} .
$$

Here, $Y_{i j d t}$ is an indicator that turns on if senior $i$ was discharged from nursing home $j$ on day $d$ of her stay. The key parameters of interest are $\gamma_{k}$ which denote the partial effects for a series of indicator variables that turn on $k$ days from the onset of co-pay. We control for day of stay and day of week fixed effects, captured by $\alpha_{d}$ and $\alpha_{\text {dow }}$, respectively.

Figure D.5 presents the estimated $\gamma_{k}$ parameters normalized to average discharge probabilities observed around day 0. The graph presents clear evidence of bunching around the day seniors start facing a daily co-pay.

\footnotetext{
${ }^{41}$ See https://www.medicare.gov/coverage/skilled-nursing-facility-snf-care, last accessed September 2018.
} 


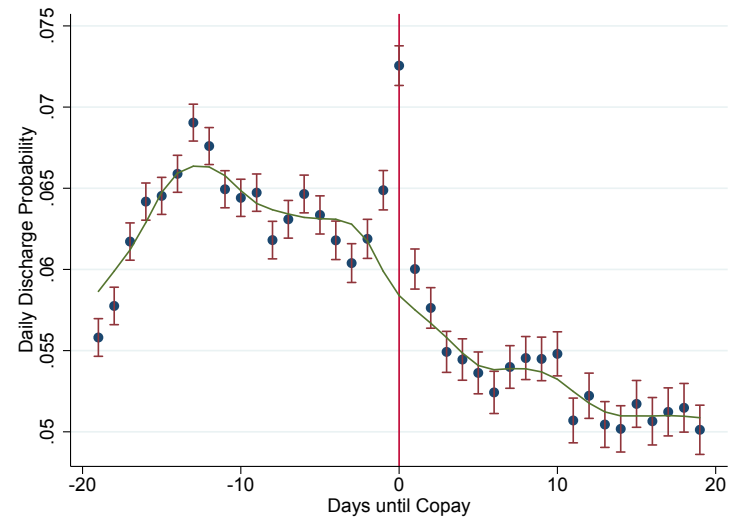

Notes: This figure plots the daily discharge probability around the day (normalized to zero) at which Medicare beneficiaries start paying a co-pay of $\$ 150$.

Figure D.5: Medicare Bunching and Co-Pay

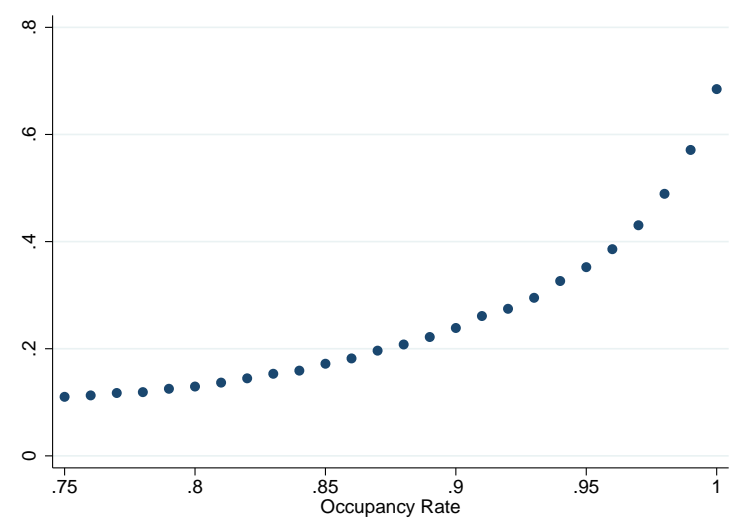

Notes: This figure plots the average weekly refill probability of an empty bed against the nursing home's occupancy rate, see equation (4) in Section 6.3 in the main text for details.

Figure D.6: Weekly Refill Probability by Occupancy Rate

\section{D.9 Provider Incentives}

Based on the discussion in Section 6.3 in the main text, Figure D.6 plots the average weekly refill probability of an empty bed on the vertical axis against the weekly occupancy rate on the horizontal axis. The figure documents a highly convex relationship highlighting an increasing option value of an empty bed at occupancies exceeding 90\%. This relationship further supports the conclusion that the increasing Medicaid discharge rates at high occupancy rates are driven by provider incentives.

As an additional test of financial incentives, we compare the discharge rates across payer types between facilities that differ in how much they benefit from replacing a Medicaid beneficiary with a private payer, which is a function of the difference between the daily rate 
that private residents pay and the average Medicaid reimbursement rate. Specifically, we calculate the average percentage difference between the two prices on the facility-year level using information on private and Medicaid rates from Pennsylvania and California (see Section 4 for details). Then we designate the quarter of nursing homes with the highest relative differences in daily rates as the high-incentive group, and the bottom quarter constitutes the low-incentive group. We expect that the relationship between occupancy and discharge rates of Medicaid residents is more pronounced in the high incentive group. The differences between private and Medicaid rates in the high-incentive group are larger than $28 \%$, and they are below $6 \%$ in the low-incentive group. Since nursing homes set their private rates freely, there is a possible concern that they may be correlated with unobserved resident characteristics, leading to an endogenous sample split. However, it is unlikely that SNFs frequently change their prices due to changes in occupancy or unobservables, and we control for facility-year effects.

Using this sample split, Figure D.7 displays estimated discharges rates by payer type and occupancy for high-incentive nursing homes on the left and low-incentive facilities on the right. Compared to the results for the whole sample in Figure 3, we find a steeper relationship between occupancy and discharge rates of Medicaid residents for the high-incentive group (left plots in Figure D.7) and a flatter relationship in the low-incentive group (right plots). The baseline discharge rates below occupancy levels of $80 \%$ are similar in the two subsamples. Hence, we find clear evidence that SNFs who have more to gain from discharging Medicaid residents are more likely to do so at high occupancy rates.

\section{D.10 Additional Health Benefits From Longer Nursing Home Stays}

In this section, we provide further details on the health effects of longer nursing home stays. Here we use the variation in financial provider incentives as a source of exogenous variation in the remaining length of stay. Specifically, a higher Medicaid discharge rate at a high occupancy rate suggests that Medicaid residents face a shorter expected remaining length of stay when the current occupancy rate is high. Building on this observation, we use regression (2) to quantify differences in health outcomes between payer types and occupancies. For example, if longer nursing home stays lead to better health outcomes, then we expect the best health outcomes for Medicaid beneficiaries at low occupancy rates because of a longer expected remaining length of stay.

One-Year Hospitalization and Mortality: We start with an analysis of the one year hospitalization rate, which is commonly viewed as a negative health outcome measure. Using the Medicare claims data, we construct an indicator variable that turns on if the resident was hospitalized within one year from the current week of stay. Figure D.8a presents the 

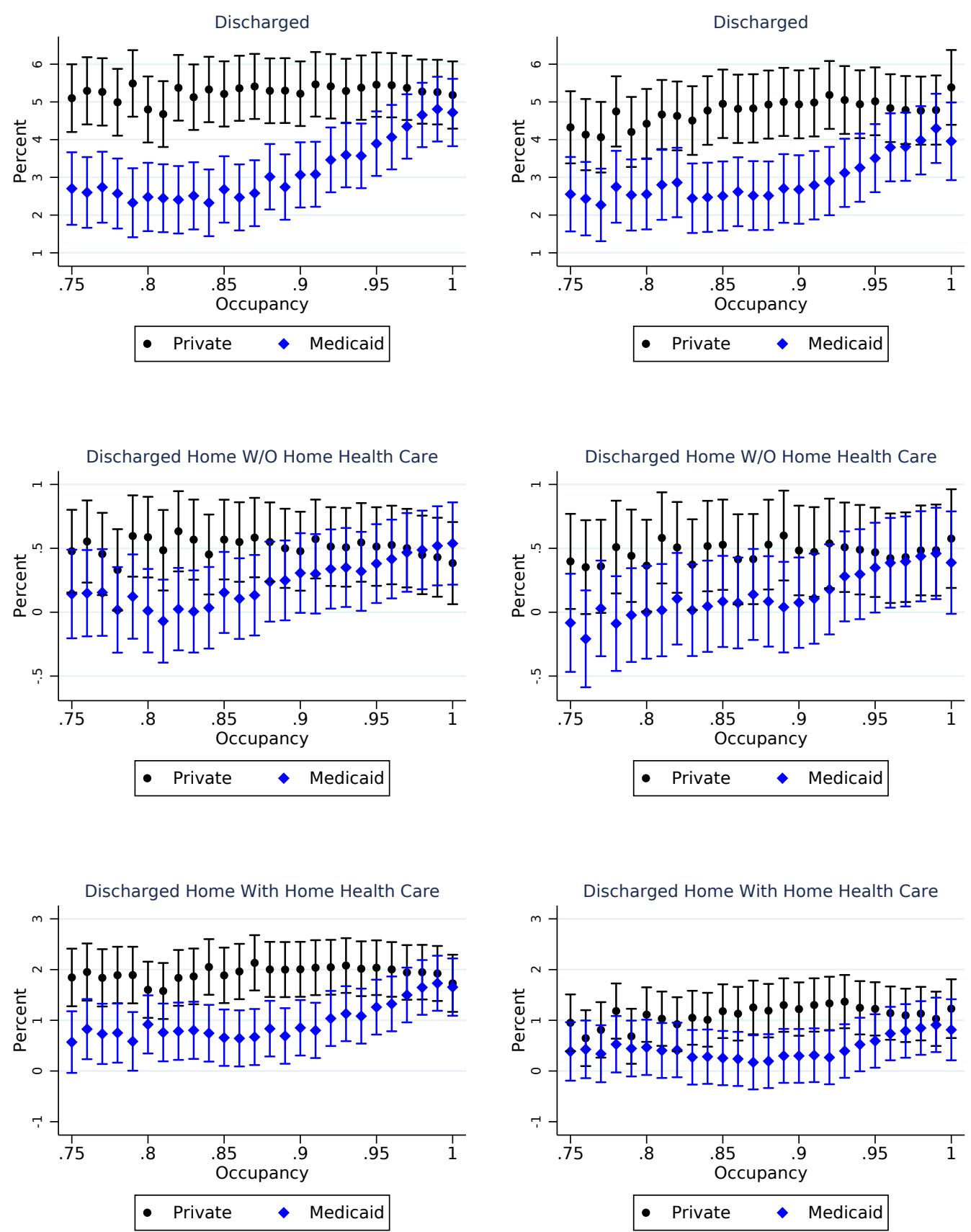

Notes: See notes for Figure 3. This figure compares overall discharges (first row), home discharges without home health care (middle row), home discharges with home health care (bottom row) between resident groups. In the left figures, we focus on SNFs with high incentives (large differences between private and Medicaid rates). In the right graphs we present the evidence for SNFs with low incentives (small differences between private and Medicaid rates). The vertical bars indicate $90 \%$ confidence intervals.

Figure D.7: Discharge Rates by Nursing Home Incentives (California and Pennsylvania) High Incentives SNFs on the Left and Low Incentive SNFs on the Right 


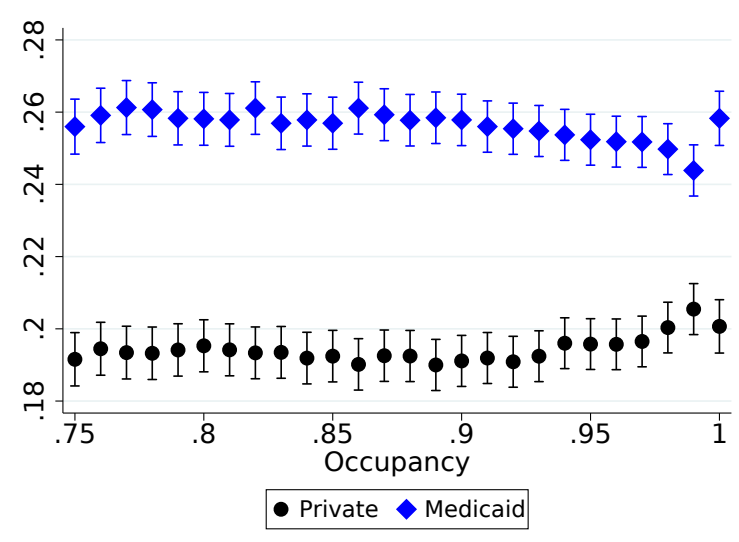

(a) 1 Year Hospitalization Rate

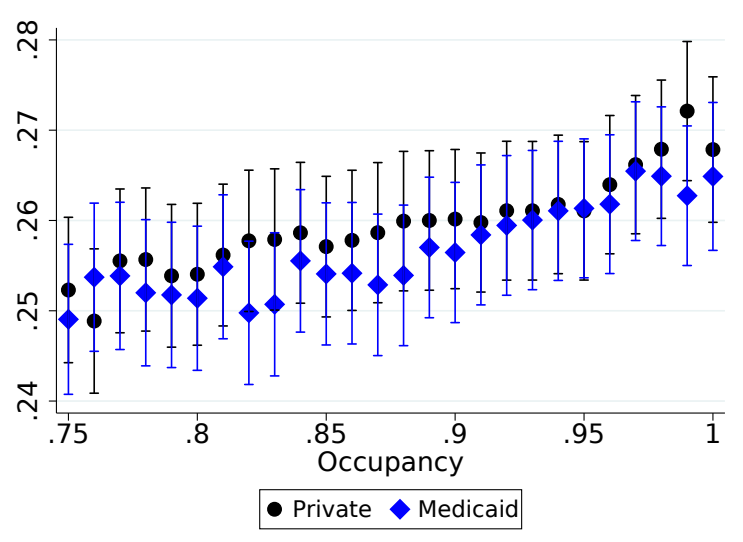

(b) 1 Year Mortality Rate

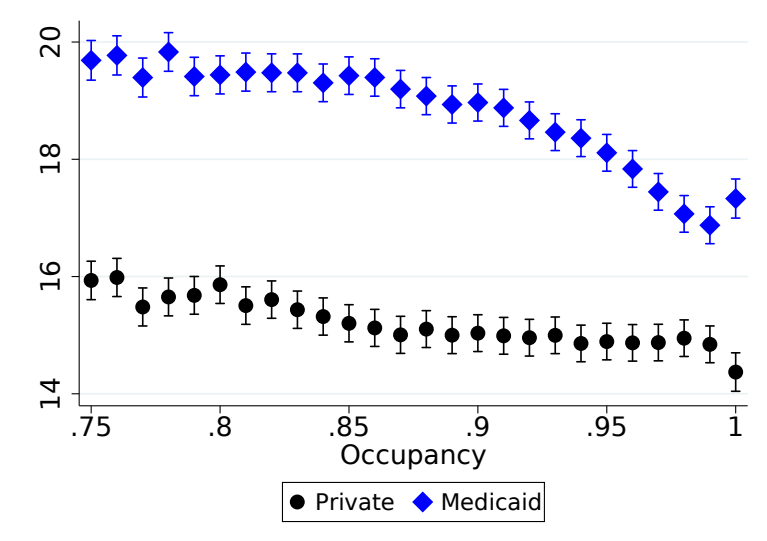

(c) Residual NH Weeks in Forthcoming Year

Notes: See notes for Figure 3. Figures D.8a, Figure D.8b, and Figure D.8c are "conceptually" analogues to 3 and plot the effects on one-year hospitalization and mortality, and the residual nursing home weeks in the upcoming 52 weeks, respectively. The vertical bars indicate $90 \%$ confidence intervals.

Figure D.8: Home Discharge Rates and Health Outcomes by Payer Type and Occupancy estimated effects of occupancy interacted with payer type. The figure points to a decline in the Medicaid one-year hospitalization rate at higher occupancy rates. In contrast, the evidence for private payers points, if anything to a small increase.

The third panel of Table D.1 summarizes the average predicted hospitalization rates between payer types at low $(<80 \%)$ and high $(>90 \%)$ occupancy rates, see Figure D. 8 for more details. At low occupancies, the one year hospitalization rate equals $19.3 \%$ for private payers, which falls short of the corresponding Medicaid rate by about 6.6 percentage points, see the first column. To reconcile the difference in hospitalization rates, we first turn to the average weekly hospitalization rate conditional on living in a nursing home. Interestingly, we find a higher weekly hospitalization rate among private residents in our sample population which stands in contrast the smaller cumulative hospitalization rates. Therefore, we consider 
Table D.1: Hospitalization Rates and Residual Length of Stay

\begin{tabular}{lcccccc}
\hline & 1Year Low & 1Year High & ResWeeks Low & ResWeeks High & Weekly NH & Weekly Comm \\
& $(1)$ & $(2)$ & $(3)$ & $(4)$ & $(5)$ & $(6)$ \\
\hline Private & $19.3 \%$ & $19.6 \%$ & 34.7 & 33.9 & $1.27 \%$ & $0.41 \%$ \\
Medicaid & $25.9 \%$ & $25.2 \%$ & 38.6 & 37.0 & $0.89 \%$ & $0.90 \%$ \\
\hline
\end{tabular}

Notes: The table hospitalization rates and the residual length of stay between payer types. Columns (1) and (2) present the one-year hospitalization rate at occupancies below $80 \%$ and above $90 \%$, respectively. Columns (3) and (4) display the residual weeks (within the next 52 weeks) spent in nursing home care at occupancies below $80 \%$ and above $90 \%$, respectively. Columns (5) and (6) display the weekly hospitalization rate conditional on living in nursing home and community care, respectively.

two alternative explanations for the difference in the cumulative hospitalization rate.

First, shorter nursing home stays among private payers may lead to lower cumulative hospitalization rates if longer nursing home stays have an adverse effect on patient health. Second, differences in weekly hospitalization rates among seniors in home care may reconcile differences in cumulative hospitalization rates which may span a significant period outside of nursing home care. This possibility is supported by the observed differences in weekly hospitalization rates among seniors who were just discharged to the community, see the sixth column. We reconcile the lower hospitalization rates for private payers in the community with better access to alternative health care providers.

To test for the first explanation and to assess the relative importance in explaining the different cumulative hospitalization rates, we exploit the variation in the length of nursing home stays due to changes in provider incentives. Columns 3 and 4 summarize the point estimates from Figure D.8c into average residual length of stay between private payers and Medicaid beneficiaries at low and high occupancy rates, respectively. To isolate the effect of provider incentives, we construct a simple difference-in-difference estimate which subtracts changes in the residuals weeks of care among private payers from changes among Medicaid beneficiaries between high and low occupancies. This yields $(37-38.6)-(33.9-34.7)=-0.8$ suggesting that provider incentives lead to a 0.8 week reduction in the length of Medicaid stays at high occupancies. ${ }^{42}$

Applying the difference-in-differences estimator to one year hospitalization rates, outlined in columns 1 and 2 , we find $(25.2 \%-25.9 \%)-(19.6 \%-19.3 \%)=-1 \%$. This suggests that

\footnotetext{
${ }^{42}$ This difference is smaller than our simulated difference in Section 8 for several reasons. First this estimate only considers a 52 week time window ignoring potential changes in the discharge timing for longer stays. Second, the occupancy rate varies randomly over the considered 52 week time horizon. Mean reversion in occupancy over time will lead to smaller differences in the residual length of stay. Third, payer types vary over time. Several private payers transition to Medicaid over the 52 week time horizon. Hence, the reduction in the residual length of stay among private payers at high occupancy rates may come from individuals who transition to Medicaid.
} 
each additional week of nursing home care increases the one year hospitalization rate by $1 / 0.8=1.25$ percentage points. This points to an adverse effect of longer nursing home stays on patient health. Returning to the differences between payer types at low occupancy rates, we find that differences in residual length of stay can explain a difference of $3.9 \cdot 1.25=4.9$ percentage points. In other words, $4.8 / 6.6=74 \%$ of the cross-sectional differences can be explained by differences in patient incentives.

We also consider the effects of longer nursing home stays on the one-year mortality. Here, we do not find significant differences at low and high occupancy rates between payer types, see Figure D.8b.

Health Profiles at Discharge: In addition to mortality and hospitalizations, we consider six health outcomes from residents' last health assessments before discharge: the CMI, ADL limitations, and indicators for clinical complexity, depression, impaired cognition, and behavioral problems. ${ }^{43}$ For all health outcomes, a higher value implies that the resident is less healthy. If longer nursing home stays of Medicaid residents improve their health, it should be reflected in these measures. ${ }^{44}$ In particular, we would expect to see a level difference between private and Medicaid residents with the latter being healthier. Moreover, we would expect that the health outcomes of Medicaid residents start to get relatively worth above 90\% occupancy since their discharge rates increase above that threshold.

Overall, Figure D.9 does not provide any evidence for health benefits of longer nursing home stays. First, Medicaid residents have worse health outcomes for all but one measure (behavioral problems) across all occupancy rates than private residents. Note that this finding is in contrast to the results in Figure D.3. That is, Medicaid residents have worse health outcomes at discharge although they are positively selected on the same measures during their stay. Second, we do not find any changes in the relationship between occupancy and health outcomes of Medicaid beneficiaries around $90 \%$ occupancy that would correspond to the kink in the discharge rate observed in Figure 3. Hence, we can reject that longer SNF stays leads to better health outcomes based on the predictions above. While there are some systematic differences between payer types - most notably the divergence in clinical complexity rates at higher occupancies - there does not seem to be a connection between the observed change in discharge rates and these health outcomes. Overall, we find no evidence suggesting that the longer nursing home stays at lower occupancies lead to any health benefits among Medicaid residents. This provides further evidence that extended

\footnotetext{
${ }^{43}$ In Figure D.3 we use the same outcomes to analyze health-related selection. However, in those regression, we use current health outcomes whereas we use health at or close to discharge in the present analysis.

${ }^{44}$ Health improvements among SNF residents are relative. We do not expect to see absolute improvements in health status, but we rather interpret the absence of worsening health status as evidence for "health benefits" of longer nursing home stays.
} 
stays among Medicaid beneficiaries contribute to LTC overspending.

\section{E Endogenous Occupancy Rates}

In the counterfactual analysis, we take endogenous changes in occupancy rates into account, which in turn affect provider discharge efforts. To this end, we divide the nursing home into two wings. The additional (external) wing allows us to incorporate admissions and discharges among residents that were excluded from the estimation sample but also affect overall occupancy. These include the $22 \%$ of nursing home stays, who were initially covered by Medicare, and an additional $22 \%$ of stays that were initially covered by Medicaid. We treat these admissions and discharges as exogenous to the counterfactual policy changes. For the study population (nursing home wing) of interest, we take observed weekly admissions as exogenous, and use our structural model to predict discharge rates under alternative policy regimes.

We calibrate admissions and discharges in the external wing to match observed changes in occupancy rates conditional on observed admissions and the estimated discharge strategies in the focal wing of interest. Specifically, we consider a nursing home of $b$ beds and simulate occupancy changes in the focal wing of interest. To this end, we draw a sequence of shocks,

$\epsilon^{s}=\left\{\epsilon_{\text {occ }}^{s}, \epsilon_{\text {arr }}^{s}, \epsilon_{\phi}^{s}, \epsilon_{\rho}^{s}, \epsilon_{d i s}^{s}\right\}$ for each simulation iteration $s \in 1, \ldots, S$. The first shock $\epsilon_{\text {occ }}^{s}$ determines the change in occupancy rate for the entire nursing home. In combination with the occupancy transition matrix $\Theta\left(o c, o c^{\prime}\right)$, this shock specifies the occupancy for the next simulation draw (or next week) $o c^{s+1}$ conditional on today's occupancy rate, $o c^{s}$.

The remaining shocks govern admissions, payer type changes, and discharges in the focal wing of interest. $\epsilon_{\text {arr }}^{s}$, in conjunction with the arrival process outlined in Figure 2c, determines the number of new arrivals. $\epsilon_{\phi}^{s}$ and $\epsilon_{\rho}^{s}$ specify, in combination with $\phi$ and $\rho$ in Table 3 , the payer type composition of new and previously admitted residents. Finally, $\epsilon_{d i s}^{s}$, in combination discharge probabilities by occupancy rate and payer type, see Figure 6, specify the number of discharged residents.

Finally, we calibrate net changes in the number of residents in the external wing to match the overall change in the occupancy rate as a result of shock $\epsilon_{o c c}^{s}$. For instance, suppose we started out with 90 occupied beds at time $s$ in the entire nursing home and that $\epsilon_{\text {occ }}^{s}$ implied a net increase to 92 occupied beds by $s+1$. Furthermore, suppose that the remaining shocks implied that the number of occupied bed in the focal wing of interest decreased from 38 to 37. Then we would assume a net increase of $\Delta_{\text {ext }}^{s}=3$ seniors in the external wing to reconcile to overall increase from 90 to 92 . This procedure generates a sequence of resident changes in the external wing $\left\{\Delta_{\text {ext }}^{s}\right\}$ for $s \in 1, \ldots, S$.

In the counterfactual analysis, we hold the sequence of shocks to the focal wing and 

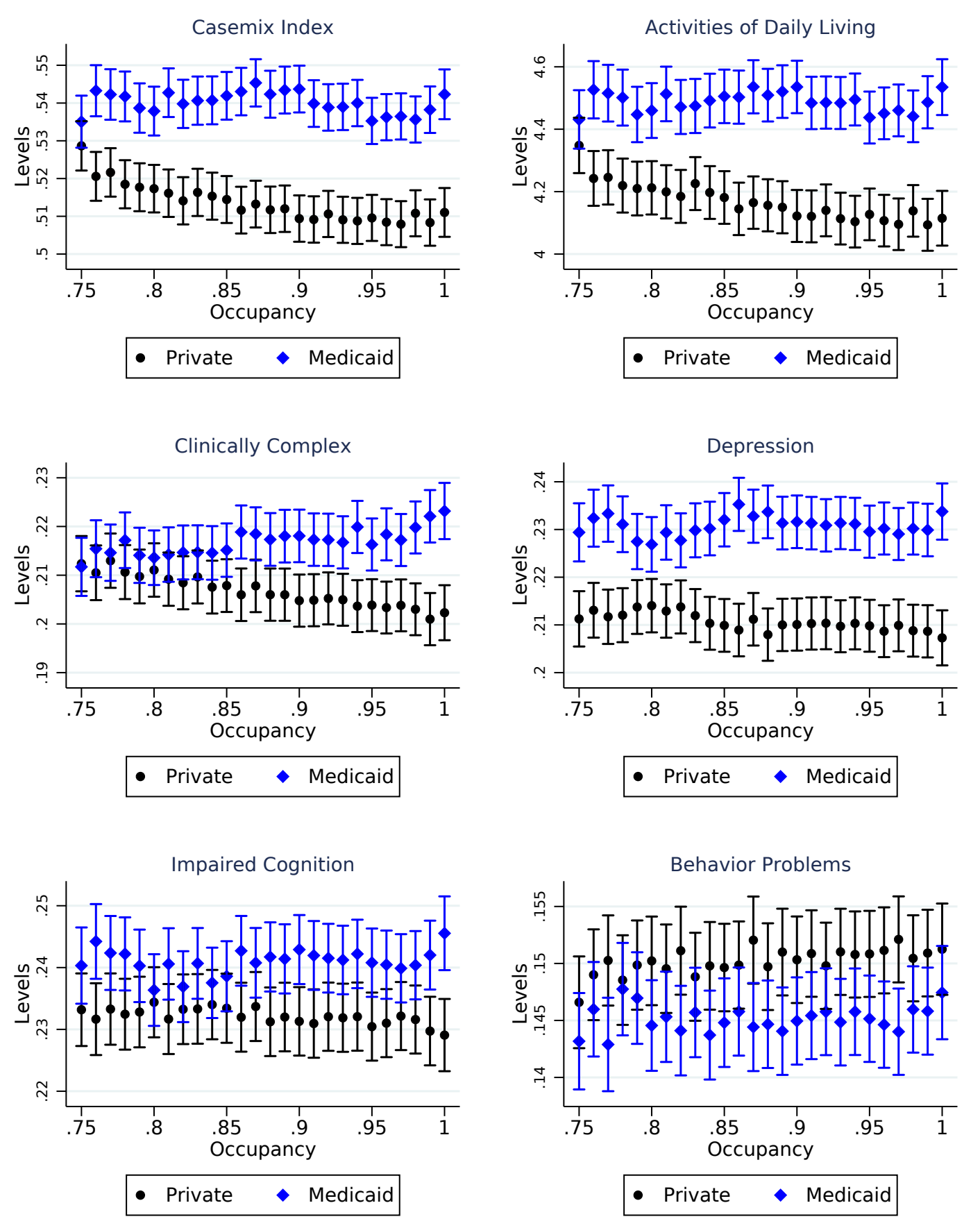

Notes: See notes for Figure 3. This figure compares the health profile at the time of discharge between payer types and occupancy rates. The resident's health status is decreasing in each health measure. The CMI is a summary measure of long term care needs, calculated based on methodology 5.01, and normalized to 1. The remaining health measures are direct inputs to the CMI formula and provide more granular information on cognitive and physical disabilities. The vertical bars indicate $90 \%$ confidence intervals.

Figure D.9: Health Profiles at Time of Discharge by Payer Type and Occupancy 
resident changes in the external wing, $\epsilon^{s}=\left\{\epsilon_{\text {arr }}^{s}, \epsilon_{\phi}^{s}, \epsilon_{\rho}^{s}, \epsilon_{\text {dis }}^{s}, \Delta_{\text {ext }}^{s}\right\}$ for $s \in 1, \ldots, S$, fixed. Importantly, we can now ignore the sequence of occupancy shocks, $\epsilon_{o c c}^{s}$. Of course, absent any policy changes, we are able to replicate the overall occupancy rate changes by inverting the strategy discussed in the previous paragraph that identified the sequence $\Delta_{\text {ext }}^{s}$. In the counterfactual analysis, we document changes in the discharge policies, see Figure 6, which we use to simulated a new sequence of overall occupancy rates. We summarize the mean occupancy rate over the simulation draws in the third row of Table 4.

\section{F Robustness to Structural Analysis}

\section{F.1 Subsample Analyses}

In this section, we provide additional robustness checks by estimating the structural model described in Section 7 on various subsamples. Table F.1 contains the parameter estimates $\{\alpha, \beta, \kappa, m c\}$ as well as the implied provider and patient elasticities of simulated length of stay with respect to a change in financial incentives. For comparison purposes, the first row repeats the baseline estimates from the first column of Table 3.

First, we split the sample by patient financial incentives. Specifically, we restrict the sample to California and Pennsylvania where price data is available. We designate nursing homes with a private rate above and below the state-specific median as high and low patient incentive homes, respectively. The estimates in the second and third rows of Table F.1 show that the resident parameters $\beta$ and $\kappa$ are slightly larger in the high-incentive sample, but the differences are not statistically significant as indicated by overlapping confidence intervals. Comparing resident elasticities reveals that the elasticity in the low-incentive sample is larger in absolute value, which is due to the fact that the financial incentive is smaller by definition. ${ }^{45}$

Next, we consider forward-looking patient behavior as defined in Section 6.2.2. Restricting the sample accordingly does not substantially change the estimated structural parameters. In particular, the implied patient elasticity only increases slightly, indicating that forward-looking patients are not driving our results. Finally, we estimate the structural model using the discharge profile that controls for occupancy at admission, see Section 6.3.3. As the last row of Table F.1 shows, the parameter estimates do not differ significantly from the baseline result and the provider elasticity is very similar. Hence, our structural model estimates are also robust to the possibility that providers cream-skim at admission.

To show how these different sets of parameter estimates affect our policy counterfactuals,

\footnotetext{
${ }^{45} \mathrm{We}$ find the same pattern in comparing provider elasticities in the high and low provider incentives in Table 3.
} 
Table F.1: Structural Parameter Estimates, Robustness

\begin{tabular}{lcccccc}
\hline & $\alpha$ & $\beta$ & $\kappa$ & $m c$ & $\epsilon^{\text {res }}$ & $\epsilon^{\text {SNF }}$ \\
\hline Baseline & 0.0314 & 0.372 & 0.116 & 77 & 0.525 & 1.62 \\
& {$[0.0267,0.0326]$} & {$[0.34,0.504]$} & {$[0.00712,0.14]$} & {$[77,77.4]$} & & \\
Low Patient Incentives & 0.0328 & 0.386 & 0.0766 & 104 & 0.619 & 1.55 \\
& {$[0.0264,0.0352]$} & {$[0.325,0.418]$} & {$[0.059,0.0831]$} & {$[88,117]$} & & \\
High Patient Incentives & 0.0396 & 0.405 & 0.0854 & 92.1 & 0.38 & 2.09 \\
& {$[0.0338,0.0421]$} & {$[0.323,0.45]$} & {$[0.0682,0.113]$} & {$[71,154]$} \\
Forward-Looking Pat. & 0.0333 & 0.417 & 0.125 & 103 & 0.654 & 1.49 \\
& {$[0.029,0.0444]$} & {$[0.333,0.441]$} & {$[0.113,0.179]$} & {$[96,201]$} \\
Controlling for Adm. Occ. & 0.035 & 0.392 & 0.0769 & 110 & 0.407 & 1.69 \\
& {$[0.0298,0.0386]$} & {$[0.335,0.426]$} & {$[0.069,0.0902]$} & {$[94,122]$} \\
Setting $u^{P}=3.5$ & 0.0346 & 0.365 & 0.05 & 74 & 0.208 & 1.43 \\
& {$[0.0305,0.0440]$} & {$[0.351,0.578]$} & {$[-0.103,0.0854]$} & {$[49,88]$} & & \\
\hline
\end{tabular}

Notes: The table shows the same estimated coefficients as Panel $C$ of Table 3 for various subsamples. The other parameters are as shown in Panels $A$ and $B$ of Table 3 except for the parameters in the last row where we set the utility levels to $u^{P}=5, u^{M}=6.5$.

we report simulated Medicaid length of stay in Table F.2. While the predicted length of stay under actual incentives is shorter in the subsamples than in our baseline results, the relative changes in length of stay under the policy counterfactuals are similar. In particular, length of stay declines the most under the voucher counterfactual, followed by the change in the reimbursement schedule that incorporates an up-front payment to nursing homes. The top-up counterfactual leads to an increase in length of stay under the subsamples as well as the baseline.

Finally, we consider the case where the patient elasticity is close to 0.2 , the level commonly found in the literature, see Section 8.3. We find that by setting private payers' gross utility from nursing home care $u^{P}=3.5$ while keeping $u^{M}=5$, we obtain a patient elasticity of 0.208, see the last row of Table F.1. The effort parameters and marginal cost of care do not change significantly from the baseline in this scenario, but the price coefficient $\kappa$ is about half as large as before. As a result of the largely unchanged provider parameters, the provider elasticity is only slightly smaller as in the baseline, but relative to the patient elasticity it becomes much larger.

In line with a lower patient elasticity, we find that the voucher counterfactual is not effective in reducing length of stay but on the contrary leads to stays that about one week longer on average, see the last row of Table F.2. Figure F.1 shows the corresponding simulated discharge profiles. Since the voucher eliminates the provider's incentives to discharge Medicaid residents at high occupancy rates, the Medicaid discharge profile is flat. At the same time, 
Table F.2: Simulated Length of Stay and Cost Savings Under Policy Counterfactuals, Robustness

\begin{tabular}{lcccc}
\hline & Actual & Voucher & Top-Up & 1\% Front \\
\hline Baseline & 31.32 & 23.64 & 35.13 & 30.28 \\
Low Patient Incentives & 27.43 & 23.62 & 31.75 & 25.34 \\
High Patient Incentives & 26.29 & 22.61 & 31.71 & 23.52 \\
Forward-Looking Pat. & 29.52 & 20.61 & 33.85 & 26.63 \\
Low Provider Incentives & 24.75 & 21.7 & 25.96 & 18.23 \\
High Provider Incentives & 21.61 & 20.15 & 28.84 & 20.24 \\
Controlling for Adm. Occ. & 26.44 & 23.34 & 31.53 & 24.47 \\
Setting $u^{P}=3.5$ & 31.62 & 32.67 & 37.13 & 29.86 \\
\hline
\end{tabular}

Notes: The table summarizes the length of stay for Medicaid residents in weeks the three counterfactual policy experiments for different samples.

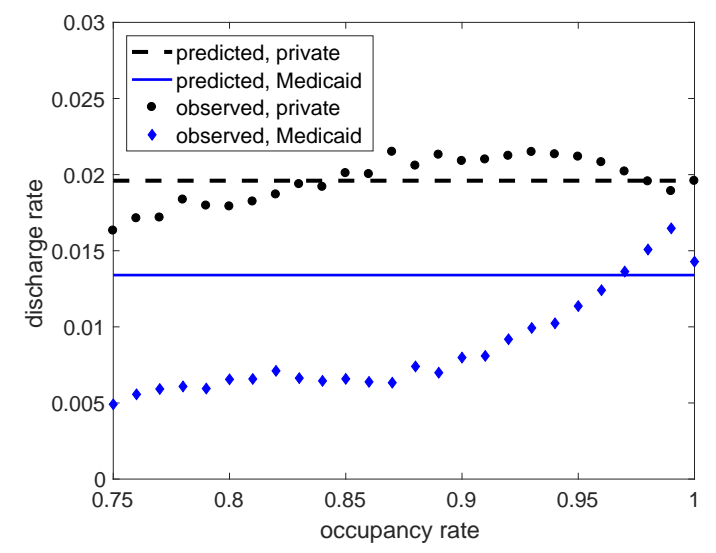

Notes: This figure is the analogue to Figure $6 b$ when Medicaid beneficiaries' utility from nursing home care is set to 6.5 .

Figure F.1: Simulated Discharge Rates Under the Voucher Counterfactual When $u^{P}=3.5$

the lower price coefficient $\kappa$ implies that Medicaid residents react less to an increase in the out-of-pocket price and therefore do not increase their own discharge efforts as much as in our baseline. In contrast, moving $1 \%$ of the daily Medicaid reimbursement to an up-front payment reduces the length of Medicaid stay by $5.6 \%$ versus 3.3\% under the baseline. Hence, if we impose a patient elasticity in line with the existing literature, we find that changing provider incentives is even more effective in shortening nursing home stays among Medicaid beneficiaries compared to changing patient incentives. 
Table F.3: Structural Parameter Estimates, Cost of Effort Function

\begin{tabular}{lccc}
\hline & & Coeff. & Conf. Int. \\
\hline SNF Effort Parameter & $\alpha$ & 1 & \\
Resident Effort Parameter & $\beta$ & 1 & \\
Linear SNF Cost Parameter & $\gamma_{1}$ & 5.139 & {$[4.818,5.419]$} \\
Quadratic SNF Cost Parameter & $\gamma_{2}$ & 1111 & {$[979,1111]$} \\
Resident Cost Scale Parameter & $\gamma_{3}$ & 0.00624 & {$[0.00524,0.00730]$} \\
Resident Price Coefficient & $\kappa$ & 0.0942 & {$[0.0671,0.108]$} \\
Marginal Cost of Care & $m c$ & 62 & {$[55,73]$} \\
\hline SNF Elasticity & $\epsilon^{S N F}$ & 1.615 & \\
Resident Elasticity & $\epsilon^{r e s}$ & 0.761 & \\
\hline
\end{tabular}

Notes: The table show estimated structural parameters and bootstrap confidence intervals as well as implied provider and patient elasticities for the alternative model specification that assumes cost of effort functions $c^{S N F}(e)=\gamma_{1} e+\gamma_{2} e^{2}$ for providers and $c^{r e s}(e)=\gamma_{3}\left(\gamma_{1} e+\gamma_{2} e^{2}\right)$ for patients.

\section{F.2 Estimating the Cost of Effort Function}

When estimating the structural model described in Section 7, we assume that discharge effort $e$ exerted by provider and patient increases the probability of a home discharge by $\alpha$ and $\beta$, see equation (5), and the cost of effort is $c(e)=e^{2}$ for both sides of the market. We then estimate $\alpha$ and $\beta$. Here, we consider an alternative model specification, in which we normalize the the marginal effect of provider and patient effort on the discharge probability to one and estimate the parameters of the cost of effort function. In particular, we assume that cost of effort is $c^{S N F}(e)=\gamma_{1} e+\gamma_{2} e^{2}$ for providers and $c^{r e s}(e)=\gamma_{3}\left(\gamma_{1} e+\gamma_{2} e^{2}\right)$ for patients. That is, the curvature of the cost of effort function is the same for both agents up to a scale factor $\gamma_{3}$. This assumption is necessary because we can only estimate the curvature of the provider cost function, which is identified from the Medicaid discharge profile above the kink point $o c^{*}$.

With this functional form assumption for the cost of effort function, the patient's optimal effort becomes

$$
e^{r e s, *}(\tau, \eta)=\max \left\{\frac{W(\tau, D=1, \eta)-W(\tau, D=0, \eta)-\kappa \gamma_{3} \gamma_{1}}{2 \kappa \gamma_{3} \gamma_{2}}, 0\right\}
$$

which is equivalent to equation (8) using a different functional form for the cost function. The provider's value function is given by the Bellman equation (10) with the cost of effort function replaced by $\gamma_{1} e+\gamma_{2} c^{2}$. We estimate the alternative model as before by minimizing 
the sum of squared differences between the private and Medicaid home discharge profiles predicted by the model and those estimated from the data (and depicted in Figure 3). In addition to the cost parameters, $\gamma_{1}, \gamma_{2}$, and $\gamma_{3}$ we also estimate the patient price coefficient, $\kappa$, and the marginal cost of providing LTC per patient and day, $m c$. The other parameters of the model are as shown in panels A and B of Table 3.

Table F.3 reports the parameter estimates along with bootstrap confidence intervals with $B=99$. While the provider cost parameters $\gamma_{1}$ and $\gamma_{2}$ do not have an intuitive interpretation, the scale parameter $\gamma_{3}$ indicates that patients' effort is about $0.6 \%$ as costly as for providers. This difference is consistent with our baseline results reported in Table 3 that show providers reacting to financial incentive more elastically than patients. Using the alternative model specification, we find elasticities of 1.62 and 0.76 for providers and patients. These estimates are similar in magnitude to our baseline results. In addition, we estimate a very similar for $\kappa$ and a slightly smaller $m c$, thereby providing further robustness to our main structural results. 